\title{
Alguns problemas de quantização em teorias com fundos não-abelianos e em espaços-tempo não-comutativos
}

\author{
Rodrigo Fresneda
}

Tese de Doutorado apresentada ao Instituto de Física da USP para a obtenção do grau de doutor em ciências.

Orientador: Prof. Dr. Dmitri Maximovitch Guitman (IFUSP)

\author{
Banca examinadora: \\ Prof. Dr. Dmitri Maximovitch Guitman (IFUSP) \\ Prof. Dr. Josif Frenkel (IFUSP) \\ Prof. Dr. Fernando Silveira Navarra (IFUSP) \\ Prof. Dr. Bruto Max Pimentel Escobar (IFT/ UNESP) \\ Prof. Dr. Antonio Edson Gonçalves (UEL)
}

São Paulo 


\section{Resumo}

Esta tese tem por base três artigos publicados pelo autor e colaboradores. O primeiro artigo trata do problema da quantização de modelos pseudoclássicos de partículas escalares em campos de fundo não-abelianos, cujo foco é a dedução desses modelos pseudo-clássicos usando métodos de integral de trajetória. O segundo artigo investiga a possibilidade de realizar modelos de gravitação dilatônica em variedades não-comutativas em duas dimensões. Para tanto, vale-se de um método de análise de vínculos e simetrias especialmente desenvolvido para gravitação não-comutativa em duas dimensões. O terceiro artigo discute modelos renormalizáveis em espaços-tempo não-comutativos com parâmetro de não-comutatividade bifermiônico em quatro dimensões. 


\begin{abstract}
This thesis is based on three published papers by the author and co-authors. The first article treats the quantization problem of pseudoclassical models of scalar particles in non-Abelian backgrounds, which aims at deriving these models using path-integral methods. The second article examines the possibility of realizing dilaton gravity models in noncommutative two-dimensional manifolds. It relies upon a method of analysis of constraints and symmetries especially developed for non-commutative dilaton gravities in two dimensions. The third article discusses renormalizable models in noncommutative spacetime with bifermionic noncommutative parameter in four dimensions.
\end{abstract}




\section{Agradecimentos}

Ao meu orientador professor Dr. Dmitri Gitman e ao professor Dr. Dmitri Vassilevich pela colaboração e ajuda.

Aos meus inúmeros amigos no IFUSP.

À Suiane.

À Fapesp pelo suporte financeiro. 


\section{Prefácio}

Esta tese tem como base os seguintes três artigos publicados pelo autor e colaboradores,

- 'Pseudoclassical description of scalar particle in non-Abelian background and path-integral representations', com D. M. Gitman, publicado em Int.J.Mod.Phys. A23:835-853 (2008);

- 'Stability of a noncommutative Jackiw-Teitelboim gravity', com D.V. Vassilevich e D. M. Gitman, publicado em Eur.Phys.J. C47:235-240 (2006);

- 'Nilpotent noncommutativity and renormalization', com D.V. Vassilevich e D.M. Gitman, publicado em Phys.Rev. D78:025004 (2008).

O primeiro artigo trata do problema da quantização de modelos pseudoclássicos de partículas escalares em campos de fundo não-abelianos, e seu foco é a dedução desses modelos pseudo-clássicos usando métodos de integral de trajetória.

O segundo artigo investiga a possibilidade de realizar modelos de gravitação dilatônica em variedades não-comutativas em duas dimensões. Para tanto, vale-se de um método de análise de vínculos e simetrias especialmente desenvolvido para gravitação não-comutativa em duas dimensões.

O terceiro artigo discute modelos renormalizáveis em espaços-tempo não-comutativos com parâmetro de não-comutatividade bifermiônico em quatro dimensões.

Os três artigos são peças independentes, e assim são apresentados nesta tese. Pelo fato de dois deles estarem ambientados em espaços-tempo não-comutativos, faz-se uma breve introdução histórica sobre geometria não-comutativa na física. Também consta na introdução uma breve discussão sobre a quantização de modelos de partícula, e em particular, da partícula em espaçostempo não-comutativos. 


\section{Sumário}

1 Introdução $\quad \mathbf{4}$

1.1 Breve história da geometria não-comutativa na física . . . . . . . . . . . . . 4

1.2 Modelos pseudoclássicos, representação de propagadores e a partícula com coorde-

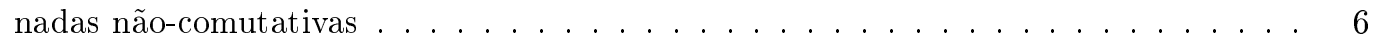

2 Partícula escalar num campo de fundo não-abeliano 9



2.2 Representações dos propagadores . . . . . . . . . . . . . . . . . . . . 10

2.3 Integral de trajetória na representação por estados coerentes . . . . . . . . . . . . . 14

2.3.1 Integral de trajetória . . . . . . . . . . . . . . . . . . . . . 14

2.3.2 Ação pseudoclássica . . . . . . . . . . . . . . . . . . . . 16

2.4 Integral de trajetória na representação da álgebra de Clifford . . . . . . . . . . . 20

2.4 .1 Integral de trajetória . . . . . . . . . . . . . . . . . . 20

2.4 .2 Ação pseudoclássica . . . . . . . . . . . . . . . . . . . 23

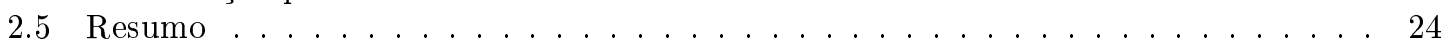

3 Estabilidade da teoria de gravitação de Jackiw-Teitelboim não-comutativa 25

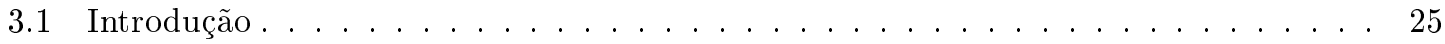

3.1 .1 Gravitação em 2 e 3 dimensões . . . . . . . . . . . . . . . . . . . . . . 27

3.1 .2 Modelo de Jackiw-Teitelboim . . . . . . . . . . . . . . . . . . . 28

3.1 .3 Gravitação dilatônica . . . . . . . . . . . . . . . . . . . . . . . . 30

3.2 Gravitação não-comutativa de Jackiw-Teitelboim . . . . . . . . . . . . . . . . . 31

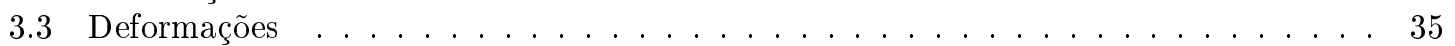

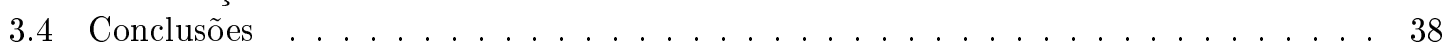

4 Não-comutatividade nilpotente e renormalização 40

4.1 Introdução . . . . . . . . . . . . . . . . . . . . . . 40

4.2 Um modelo de campo escalar . . . . . . . . . . . . . . . . . . 41

4.3 Eletrodinâmica não-comutativa com parâmetro bifermiônico . . . . . . . . . . . . . 44

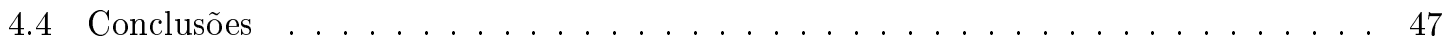

A Ordenamento de Weyl de operadores e funções na álgebra de Berezin 49

B Notação e identidades úteis ao capítulo $2 \quad 51$ 


\section{Capítulo 1}

\section{Introdução}

\subsection{Breve história da geometria não-comutativa na física}

Em 1946 Snyder [1] propõe um novo modelo para o espaço-tempo, em que a energias suficientemente altas, a continuidade do espaço-tempo clássico dá lugar a uma noção de espaço-tempo fragmentado em células de tamanho mínimo, onde não há a idéia de ponto. Nesta nova escala fundamental as coordenadas do espaço-tempo não são mais parâmetros contínuos, como na teoria quântica usual, mas operadores hermitianos que não comutam entre si:

" $x, y, z$, e $t$ são operadores hermitianos para as coordenadas do espaço-tempo de um referencial de Lorentz particular; o espectro de cada um desses operadores $x, y, z$, e $t$ é composto dos possíveis resultados da medida da quantidade correspondente; os operadores $x, y, z$, e $t$ devem ser tais que o espectro dos operadores $x^{\prime}, y^{\prime}, z^{\prime}$ e $t^{\prime}$ formados por combinações lineares de $x, y, z$, e $t$, que deixam a forma quadrática (o elemento de linha do espaço de Minkowski) invariante, seja o mesmo que o espectro de $x, y, z$, e $t . "$

Como consequência direta da não comutatividade dos operadores correspondentes às coordenadas do espaço-tempo, resulta a impossibilidade de diagonalizar simultaneamente esses operadores , ou seja, resulta a impossibilidade de medir com precisão arbitrária a posição de uma partícula. Por exemplo, a álgebra mais simples de operadores $\hat{x}^{\mu}$, que aqui representam os operadores hermitianos correspondentes às coordenadas do espaço-tempo, é dada em termos de uma matriz real anti-simétrica constante $\theta^{\mu \nu}$,

$$
\left[\hat{x}^{\mu}, \hat{x}^{\nu}\right]=i \theta^{\mu \nu} .
$$

As relações acima implicam as seguintes relações de incerteza:

$$
\Delta \hat{x}^{\mu} \Delta \hat{x}^{\nu} \geq \frac{1}{2}\left|\theta^{\mu \nu}\right|
$$

Logo, a distâncias da ordem de $\sqrt{\left|\theta^{\mu \nu}\right|}$, efeitos da não comutatividade das coordenadas do espaçotempo passam a ser relevantes, sinalizando o fim do modelo clássico para o espaço-tempo e o início de uma nova estrutura geométrica subjacente.

À época em que a não comutatividade foi sugerida, esperava-se que ela pudesse resolver o problema das divergências ultra-violeta da teoria quântica de campos, em particular, da eletrodinâmica quântica, problema cuja origem está no produto de distribuições num dado ponto do espaço. Ao 
impor um comprimento mínimo e eliminar o conteúdo físico da noção de ponto, surge um novo tipo de regularização, semelhante à introdução de um cut-off para os momentos, porém invariante de Lorentz - ao menos na concepção original de Snyder, em que os operadores $\hat{x}^{\mu}$ são os geradores das simetrias do espaço de de Sitter. No entanto, com o sucesso do programa de renormalização, esse caminho foi momentaneamente abandonado, e somente foi retomado na década de 1980, com os trabalhos de Connes e Woronowicz [2,3] no contexto de geometria não-comutativa, termo cunhado originalmente por von Neumann [4].

$\mathrm{O}$ interesse da comunidade de física ressurge com a aplicação da geometria não-comutativa à formulação de teorias de calibre não-abelianas [5], da gravitação [6, 7, 8, 9] e do modelo padrão de partículas [10, 11, 12], e também no entendimento do efeito Hall quântico [13].

No entanto, esse interesse renovado não se deveu propriamente à realização da promessa inicial: as teorias de campo em espaços-tempo não-comutativos não constituíram um avanço quanto à finitude e renormalizibilidade das teorias quânticas de campo. De fato, a não-localidade do produto estrela de Moyal faz com que pequenas perturbações a energias altas se propaguem longe o bastante para influenciar processos a baixas energias, dando lugar à mistura de divergências ultra-violetas e infra-vermelhas em diagramas não-planares $[14,15,16]$. Por exemplo, para a teoria $\lambda \varphi^{4}$ no $\mathbb{R}^{4}$ não-comutativo, a parte não-planar da função de dois pontos irredutível de uma partícula padece da mesma divergência ultra-violeta que seu correspondente planar, mas para momentos baixos. A presença da patologia de mistura de divergências ultra-violeta e infra-vermelha na teoria $\lambda \varphi^{4}$ no $\mathbb{R}^{4}$ não-comutativo é suficiente para estragar a renormalização da massa nesse modelo a um loop, tornando-o não-renormalizável. A almejada renormalização desse modelo só foi atingida com a modificação do propagador pela adição de um termo oscilante nos trabalhos [17, 18, 19], com o fim de respeitar a dualidade de Langmann-Szabo [20]. Depois, demonstrou-se que muitos outros modelos, apropriadamente modificados para exibir essa dualidade, são renormalizáveis, como o modelo de Langmann-Szabo-Zarembo em quatro dimensões [21], o modelo de Gross-Neveu em duas dimensões [22], e o modelo $\varphi^{3}$ não-comutativo em duas, quatro e seis dimensões [23, 24, 25].

Apesar dos avanços no desenvolvimento de estruturas diferenciáveis em geometria não-comutativa e nas inúmeras generalizações da teoria quântica de campos a espaços não-comutativos, é graças à teoria de cordas que a geometria não-comutativa deve sua popularidade. Na teoria de cordas, a geometria não-comutativa faz sua primeira aparição no trabalho [26] do Witten sobre a corda aberta. Desde então, a não-comutatividade do espaço-tempo tem surgido de forma ubíqua na teoria de cordas: no estudo não-perturbativo de dualidades, como a dualidade $T$ na compactificação toroidal [27], manifestação da geometria quântica inerente à teoria de cordas enquanto teoria de gravitação quântica; na classificação de campos de fundo por meio de teorias de Yang-Mills não-comutativas; e principalmente, na teoria de $D$-branas, que no limite de baixas energias é efetivamente descrita por coordenadas não-comutativas com valores matriciais [28]. A relação íntima entre geometria não-comutativa e regimes não-perturbativos da teoria de cordas é perfeitamente condizente com análises anteriores sobre espalhamento de cordas a altíssimas energias e sobre a escala fundamental da teoria de cordas $[29,30,31]$. A existência de um limite inferior para a medida de distâncias, da ordem da escala de comprimento intrínseca da corda, é mais um argumento a favor de uma descrição geométrica 'não-pontual' do espaço-tempo. Há de se citar, ademais, a presença de geometria não-comutativa na dinâmica quântica da corda aberta na presença de campos de fundo e de $D$-branas $[32,33,34,35,36]$.

Como ilustração simples de uma realização física de não-comutatividade do espaço, considere uma partícula carregada no plano $\mathbf{x}=\left(x^{1}, x^{2}\right)$, na presença de um campo magnético constante. 
Sua lagrangiana é

$$
L_{m}=\frac{m}{2} \mathbf{x}^{2}+\mathbf{x} \cdot \mathbf{A}, A_{i}=\frac{B}{2} \epsilon_{i j} x^{j} .
$$

No limite em que o campo magnético é muito forte, desprezamos o termo de inércia fazendo $m=0$, e ficamos com

$$
L_{0}=-\frac{B}{2} \dot{x}^{i} \epsilon_{i j} x^{j}
$$

A hamiltoniana desse sistema é proporcional aos vínculos de segunda classe $\Phi_{i}=\pi_{i}+\frac{B}{2} \epsilon_{i j} x^{j}$ e os parênteses de Dirac entre as coordenadas são

$$
\left\{x^{i}, x^{j}\right\}_{D(\Phi)}=B^{-1} \epsilon^{i j},
$$

ou seja, são uma matriz anti-simétrica constante. A quantização dessas relações de comutação, de acordo com a promoção das coordenadas $x^{i}$ a operadores $\hat{x}^{i}$ e substituição dos parênteses de Poisson por comutadores,

$$
\left[\hat{x}^{i}, \hat{x}^{j}\right]=i\left\{x^{i}, x^{j}\right\}_{D(\Phi)},
$$

produz as relações básicas (1.1) com $\theta^{i j}=B^{-1} \epsilon^{i j}$.

Algo semelhante ocorre com teoria de cordas [37]. Considere uma corda bosônica num espaço plano de métrica $g^{i j}$ na presença de um campo $B$ de Neveu-Schwarz constante ,

$$
S_{\Sigma}=\frac{1}{4 \pi \alpha^{\prime}} \int_{\Sigma}\left(g_{i j} \partial_{a} x^{i} \partial^{a} x^{j}-2 \pi \alpha^{\prime} B_{i j} \epsilon^{a b} \partial_{a} x^{i} \partial_{b} x^{j}\right),
$$

em que $\Sigma$ é a folha-mundo da corda. O segundo termo no integrando é uma derivada total, e para cordas abertas, pode ser escrito em termos de uma integral na borda da folha-mundo:

$$
S_{\partial \Sigma}=-\frac{i}{2} \oint_{\partial \Sigma} B_{i j} x^{i} \partial_{t} x^{j}
$$

em que $t$ é a coordenada em $\partial \Sigma$. No limite de baixas energias, $g_{i j} \sim \alpha^{\prime 2} \rightarrow 0$, o termo cinético principal da ação da corda desaparece, e o que resta são os graus de liberdade na borda da corda aberta. Podemos interpretar a ação $S_{\partial \Sigma}$ como a ação de uma partícula num campo magnético forte. Isto nos permite inferir que as coordenadas da corda na fronteira fazem parte de uma álgebra não-comutativa

$$
\left[\hat{x}^{i}, \hat{x}^{j}\right]=i\left(B^{-1}\right)^{i j}
$$

O limite $\alpha^{\prime} \rightarrow 0$ de fato transforma a teoria de cordas numa teoria de campos efetiva. O fato da teoria de campos não-comutativa surgir naturalmente a partir da teoria de cordas sugere fortemente que não-comutatividade do espaço-tempo é traço geral de uma teoria unificada de gravitação quântica. Também no contexto de gravitação quântica de laços [38], operadores de área calculados em superfícies que se interceptam não comutam entre si, fazendo com que a geometria espacial (em três dimensões) seja não-comutativa.

\subsection{Modelos pseudoclássicos, representação de propagadores e a partícula com coordenadas não-comutativas}

Modelos pseudoclássicos de partículas, isto é, modelos de partículas cujas coordenadas são variáveis em uma álgebra de Berezin [39], apresentam um grande interesse em vários aspectos da teoria quântica. Do ponto de vista fundamental, sua quantização conduz às equações de onda e, inversamente, 
surgem como limite (pseudo)clássico das dinâmicas quânticas correspondentes. Do ponto de vista metodológico, servem à representação de objetos da teoria quântica, tais como propagadores. Finalmente, pode-se construir a mecânica quântica inteiramente baseada em símbolos pseudoclássicos de operadores na chamada formulação de Moyal [40].

O problema da quantização de modelos de partículas relativísticas ocupa um lugar central no chamado problema de quantização, na medida em que fornece um entendimento básico dos princípios que regem a quantização dos modelos mais simples que podem ser encontrados na natureza. A quantização da partícula relativística, qualquer que seja sua massa e spin e o campo de fundo considerado, relaciona-se com o problema da construção da equação de onda e, portanto, de uma mecânica quântica para esta partícula. O artigo [41] estende a importância da quantização de modelos pseudoclássicos, mostrando que a quantização da partícula relativística pode ser consistentemente interpretada como a construção do setor de uma partícula da teoria quântica de campos correspondente, sempre que esse setor possa ser definido (i.e., quando a interação da partícula com os campos de fundo não leve à criação de partículas). É central no problema da quantização de modelos de partículas relativísticas a presença de vínculos e liberdade de calibre.

Sempre que possível, estes modelos devem ser definidos e quantizados em dimensões arbitrárias. A generalização de modelos pseudoclássicos e sua quantização em dimensões arbitrárias esbarra em dificuldades maiores no caso da partícula espinorial em dimensões ímpares, devido à redutibilidade da álgebra de Clifford. Isto quer dizer que em dimensões ímpares a quantização da partícula espinorial deve produzir estados físicos em duas representações de Weyl distintas, que correspondem às duas representações não-equivalentes da álgebra das matrizes gamma. Em $2+1$ dimensões, mostrou-se [42] que esse fato evidencia-se na presença de um vínculo bifermiônico que não pode ser fixado. É possível ainda construir modelos pseudoclássicos em interação com campos não-abelianos. O trabalho [43] apresenta um modelo assim; lá utiliza-se, no entanto, o método de quantização de Dirac. Seria portanto interessante tratar um modelo de partícula em campo não-abeliano segundo o procedimento de quantização canônica consistente, nos moldes de [41].

A representação de propagadores da teoria quântica por integrais de trajetória é um problema intimamente relacionado aos modelos pseudoclássicos. Os trabalhos [44, 45] mostram que os modelos pseudoclássicos para partículas escalar e com spin 1/2 em campo eletromagnético arbitrário podem ser extraídos de tais representações, e o trabalho [46] generaliza estes cálculos em dimensão arbitrária. Estas representações podem ainda ser generalizadas para partículas em interação com campos não abelianos $[47,48]$, sempre levando a integrais de trajetória cujas ações correspondem a modelos pseudoclássicos. A definição precisa da integral de trajetória pelo método de discretização corresponde a uma definição precisa da correspondência entre símbolos e operadores [49]. Em outras palavras, as ambiguidades de ordenamento que se encontram na quantização de funções clássicas se manisfestam igualmente na definição discreta da integral de trajetória. Como já mencionado, tais ambiguidades de ordenamento se manifestam muito claramente no problema da partícula em campo gravitacional, e assim é de se esperar que surjam problemas na definição da integral de trajetória no curso da representação de propagadores de partículas em campo gravitacional.

No contexto de espaços-tempo não-comutativos, esforços na direção da quantização de modelos de partículas ainda são incipientes, e se concentram principalmente na generalização ao caso nãocomutativo de problemas clássicos da mecânica quântica usual, tais como o oscilador harmônico [50, 51, 52], a partícula no campo central e o átomo de hidrogênio [53, 54], e também incorporando efeitos topológicos, tais como efeito Aharonov-Bohm e efeito Casimir [55, 56]; todos com o fim de obter correções quânticas oriundas da não-comutatividade das coordenadas. Também se incluem nesses esforços de obter correções quânticas da não-comutatividade abordagens de quantização via 
integral de trajetória $[57,58,59,60,61]$.

No entanto, somente em [62] há preocupação em justificar as ações clássicas empregadas para efeito de quantização, valendo-se do problema inverso de quantização: pela extração das referidas ações clássicas e pseudoclássicas das representações dos propagadores correspondentes via integral de trajetória. Descobre-se que tanto a ação hamiltoniana clássica para partícula relativística escalar, quanto a ação pseudoclássica hamiltoniana para partícula relativística espinorial, diferem de seus análogos comutativos ([45] e [39], respectivamente) pelo termo $\dot{p}_{\mu} \theta^{\mu \nu} p_{\nu} / 2 \hbar$, que impede que se obtenham diretamente por integração a forma lagrangiana das ações. A quantização de Dirac da ação pseudoclássica assim obtida recupera a equação de Dirac no espaço de Minkowski nãocomutativo, justificando a generalização do modelo de Berezin-Marinov. Por fim, em [62] também se deduz a ação clássica da partícula não-relativística pela representação da função de propagação via integral de trajetória, obtendo-se uma modificação da ação hamiltoniana clássica no $\mathbb{R}^{3}$ pelo mesmo termo que surge nas versões relativísticas, que incidentalmente é a mesma ação proposta em $[63,64,65]$ 


\section{Capítulo 2}

\section{Partícula escalar num campo de fundo não-abeliano}

\subsection{Introdução}

Teoria Quântica de Campos (TQC) com campos externos é uma boa abordagem à descrição de muitos sistemas e efeitos físicos. Se o campo externo é forte o bastante, ele tem de ser computado não-perturbativamente. Os métodos correspondentes para Eletrodinâmica Quântica (EQ) estão bem desenvolvidos e deram muitos resultados, como mostram os trabalhos [66] e as citações aí contidas. O conceito de campo externo em TQC não-abeliana é menos desenvolvido e encontra algumas dificuldades (não há uma forma de introduzir um campo externo não-abeliano que seja invariante de calibre). Entretanto, a existência indiscutível de situações físicas em que há um campo não-abeliano quantizado suficientemente forte frequentemente serve como justificativa física ao tratamento desse campo com um campo externo clássico, apesar do problema mencionado acima. Resultados interessantes e fisicamente significativos que foram obtidos nesse contexto servem como justificativa adicional. Podemos apontar os cálculos efetivos a um loop em campos externos constantes nãoabelianos $[67,68,69]$ que foram usados na construção do verdadeiro vácuo da Cromodinâmica Quântica (CQ), veja [69, 70, 71, 72]. Também se deve mencionar a descrição de transições de fase em CQ cosmológica [73], produção não perturbativa de partons a partir do vácuo por um campo clássico cromoelétrico $S U$ (3) [74] e $S U$ (2) [75], condições de fronteira e efeitos topológicos do vácuo na presença de um campo magnético inomogêneo na forma de um tubo de fluxo [76, 77], e assim por diante.

Os objetos-chave em TQC não-perturbativa (com respeito ao campo de fundo) com um campo de fundo não-abeliano são os propagadores das partículas escalar e espinorial no campo externo nãoabeliano correspondente. Soluções exatas para tais objetos permitem que se obtenha por integração resultados a um loop para várias quantidades físicas. Ademais, representações por integral de trajetória do propagador podem ser úteis à obtenção de soluções exatas, que então poderíam ser usadas em cálculos. Uma variedade de representações por integral de trajetória da partícula escalar e espinorial foram construídas e calculadas para muitos campos abelianos de fundo em [78, 48, 44, 45, $79,47,80]$. Verificou-se que tais representações também são úteis na dedução das chamadas ações pseudoclássicas para partículas espinoriais, veja [45, 79, 46]. Algumas representações por integral 
de trajetória para propagadores em campos externos não-abelianos e problemas relacionados à descrição pseudoclássica de isospin foram estudadas em [43, 81, 48].

Recordamos que uma teoria clássica para a partícula de Yang-Mills foi primeiro obtida a partir do limite clássico das equações de campo de Yang-Mills por Wong [82]. Em seguida, Chen e Dresden [83] mostraram que as equações de campo de Yang-Mills fornecem as equações de movimento para uma partícula teste com spin isotópico no mesmo sentido em que as equações de Einstein fornecem as equações para uma partícula teste massiva. Casalbuoni et.al. [81] obtiveram uma descrição lagrangiana invariante de calibre para partículas escalar e espinorial com spin isotópico, em que variáveis de Grassmann descrevem os graus de liberdade internos no nível clássico, tal que a quantização dê representações de dimensão finita do grupo de calibre. Balachandran et.al. [43] aplicaram o procedimento de quantização de Dirac a uma formulação lagrangiana pseudoclássica das partículas escalar e espinorial interagindo com um campo de calibre não-abeliano, e adicionalmente, desenvolveram um método aqui utilizado para obter as representações irredutíveis de isospin. Em [47], a estrutura de isospin do propagador da partícula relativística escalar na representação fundamental de $S U(2)$ é deduzida usando métodos desenvolvidos para o caso da partícula com spin.

Aqui volta-se mais uma vez a esses problemas para o caso da partícula escalar com isospin em vários campos externos não-abelianos. É importante frisar que um campo escalar quantizado em um campo externo não-abeliano tem sido proposto como uma explicação de confinamento em CQ por meio de uma partícula escalar massiva (dílaton) [84], e também aparece na forma de escalares fundamentais acoplados à curvatura de calibre em teoria de cordas [85].

Construíram-se representações por integral de trajetóra para o propagador da partícula escalar em duas abordagens: uma é a generalização do procedimento proposto em [47] a qualquer representação de $S U(N)$ dada em termos de matrizes de base anti-simétricas, enquanto a outra é construída usando estados coerentes fermiônicos válida para quaisquer representações de $S U(N)$. Esta última abordagem é uma modificação da representação por integral de trajetória do propagador de Dirac por meio de estados coerentes fermiônicos apresentado em [48]. Em ambos os casos nós deduzimos as ações pseudoclássicas para a partícula escalar em campos de fundo não-abelianos, e os quantizamos para demonstrar sua consistência. No apêndice, colocamos alguns detalhes técnicos e demonstrações.

\subsection{Representações dos propagadores}

Um campo escalar $\phi$ com carga não-abeliana é um campo no espaço de Minkowski $\mathcal{M}$ de métrica $\eta_{\mu \nu}=\operatorname{diag}(1,-1,-1,-1)$ com valores em um espaço de representação linear $V$ de um grupo de simetria local $G$, i.e., $\phi: \mathcal{M} \rightarrow V$. Aqui e no que segue, $G=S U(N)$, e a representação linear é uma representação matricial unitária de dimensão $n$, isto é, $\rho: S U(N) \rightarrow G L(n), V=\mathbb{C}^{n}$ $\left(\phi=\left\{\phi_{\alpha}\right\}\right.$ é uma $n$-upla de números complexos, $\left.\alpha=1, \ldots, n\right)$ e $\rho(g)$ com $g \in S U(N)$ é uma matriz unitária de ordem $n$. O potencial de calibre $\mathcal{A}_{\mu}$ é um campo em $\mathcal{M}$ com valores na álgebra de Lie $s u(N), \mathcal{A}_{\mu}: \mathcal{M} \rightarrow s u(N)$, e sua ação sobre os campos de matéria $\phi$ se dá de acordo com a representação induzida por $\rho$. A base de $s u(N)$ nessa representação é dada pelas matrizes hermitianas de traço nulo $t_{a \beta}^{\alpha}, a=1, . ., N^{2}-1$ e $\alpha, \beta=1, \ldots, n^{1}$. Desse modo, $\mathcal{A}_{\mu} \phi=A_{\mu}^{a} t_{a \beta}^{\alpha} \phi^{\beta}$,

\footnotetext{
${ }^{1}$ Como $S U(N)$ é um grupo compacto, a base pode ser escolhida tal que as constantes de estrutura sejam totalmente anti-simétricas [86],

$$
\left[t_{a}, t_{b}\right]=f_{a b}^{c} t_{c}, f_{a b}^{c} \equiv f_{[a b c]}
$$


onde $A_{\mu}^{a}(x)$ são os coeficientes de $\mathcal{A}_{\mu}$ na base $\left\{t_{a}\right\}$. A equação que define o campo escalar $\phi$ é a equação de Klein-Gordon

$$
\left(\eta_{\mu \nu} \mathcal{P}_{\mu} \mathcal{P}_{\nu}-m^{2}\right) \phi(x)=0
$$

em que $\mathcal{P}_{\mu}=i \partial_{\mu}-q \mathcal{A}_{\mu}$ é a derivada covariante. Por uma transformação de calibre local $g: \mathcal{M} \rightarrow$ $S U(N)$

$\phi(x) \mapsto g(x) \phi(x), \phi^{\dagger}(x) \mapsto \phi^{\dagger}(x) g^{-1}(x), \mathcal{A}_{\mu}(x) \mapsto g(x) \mathcal{A}_{\mu}(x) g^{-1}(x)+i q^{-1} \partial_{\mu} g(x) g^{-1}(x)$,

a derivada covariante satisfaz $\mathcal{P}_{\mu} \phi(x) \mapsto g(x) \mathcal{P}_{\mu} \phi(x)$, em que se subentende que o elemento $g$ de $S U(N)$ é dado em termos da representação $\rho$. A ação invariante por transformação de calibre local $g: \mathcal{M} \rightarrow S U(N)$ mais simples cuja lagrangiana seja quadrática e função de $\phi$ e suas primeiras derivadas $\partial_{\mu} \phi$, e cujo extremo dê a equação de Klein-Gordon é

$$
S=\int d^{4} x\left\{\eta^{\mu \nu}\left(\mathcal{P}_{\mu} \phi\right)^{\dagger}\left(\mathcal{P}_{\nu} \phi\right)-m^{2} \phi^{\dagger} \phi\right\}, \mathcal{P}_{\mu}=i \partial_{\mu}-q \mathcal{A}_{\mu}
$$

O propagador causal para a partícula escalar relativística interagindo com um campo externo $\mathcal{A}_{\mu}$ com valores na álgebra $s u(N)$ no espaço de Minkowski (em unidades naturais $\hbar=c=1$ ) é descrito pela equação

$$
\left(\mathcal{P}^{2}-m^{2}\right)_{\beta}^{\alpha} D_{\gamma}^{\beta}(x, y)=-\delta_{\gamma}^{\alpha} \delta^{4}(x-y), \mathcal{P}_{\mu}=i \partial_{\mu}-q \mathcal{A}_{\mu},
$$

No que segue consideraremos duas diferentes realizações da álgebra de Lie (2.1) de $s u(N)$. A primeira realização será em termos de operadores de criação e aniquilação definidos num espaço de Fock conveniente, e a segunda realização será em termos de geradores de uma álgebra de Clifford particular.

I. Considere um espaço de Hilbert abstrato $\mathcal{H}$ dado pelo produto direto do espaço de representação usual para a álgebra de Heisenberg, cuja base é denotada por $|x\rangle$,

$$
\begin{aligned}
& \hat{x}^{\mu}|x\rangle=x^{\mu}|x\rangle,\langle x \mid y\rangle=\delta^{4}(x-y), \int d^{4} x|x\rangle\langle x|=I, \\
& {\left[\hat{x}^{\mu}, \hat{p}_{\nu}\right]=i \delta_{\nu}^{\mu},\left\langle x\left|\hat{p}_{\mu}\right| y\right\rangle=-i \partial_{\mu} \delta^{4}(x-y)}
\end{aligned}
$$

e um espaço de Hilbert abstrato $V$ que por ora não especificamos, e que possui uma base ortonormal de vetores $|\alpha\rangle, \alpha=1, \ldots, n$, com produto interno

$$
\langle\alpha \mid \beta\rangle=\delta_{\alpha \beta}, \sum_{\alpha=1}^{n}|\alpha\rangle\langle\alpha|=I .
$$

Desse modo, o espaço de Hilbert $\mathcal{H}=H \otimes V$ possui uma base ortonormal $|x, \alpha\rangle=|x\rangle \otimes|\alpha\rangle$, e produto interno $\langle x, \alpha \mid y, \beta\rangle=\delta^{4}(x-y) \delta_{\alpha \beta}$.

Em seguida, interpretamos os operadores com índices que surgem em (2.3) como elementos de matriz de operadores em $\mathcal{H}$. De acordo com esta interpretação, o propagador $D_{\beta}^{\alpha}(x, y)$ são elementos de matriz de um operador abstrato $\hat{D}$,

$$
D(x, y)_{\beta}^{\alpha}=\langle x, \alpha|\hat{D}| y, \beta\rangle,
$$

e as matrizes $t_{a}$ sejam normalizadas a $\operatorname{tr}\left(t_{a} t_{b}\right)=1 / 2 \delta_{a b}$. 
e a base $t_{a \beta}^{\alpha}$ são elementos de matriz de operadores $\hat{t}_{a}$,

$$
\left\langle\alpha\left|\hat{t}_{a}\right| \beta\right\rangle=t_{a \beta}^{\alpha}
$$

Note que como os elementos de matriz dos operadores $\hat{t}_{a}$ satisfazem as relações de comutação da álgebra $s u(N)$, os operadores também as satisfazem:

$$
\left[t_{a}, t_{b}\right]_{\beta}^{\alpha}=f_{a b}^{c} t_{c \beta}^{\alpha} \Leftrightarrow\left[\hat{t}_{a}, \hat{t}_{b}\right]=f_{a b}^{c} \hat{t}_{c} .
$$

Por meio dos operadores acima definidos, pode-se escrever (2.3) na forma operatorial,

$$
\left(\hat{P}^{2}-m^{2}\right) \hat{D}=-I
$$

em que

$$
\hat{P}_{\mu}=-\hat{p}_{\mu}-q \hat{\mathcal{A}}_{\mu}, \hat{\mathcal{A}}_{\mu}=A_{\mu}^{a}(\hat{x}) \hat{t}_{a},\left\langle x, \alpha\left|\hat{P}_{\mu}\right| y, \beta\right\rangle=\left(i \partial_{\mu} \delta_{\alpha \beta}-q A_{\mu}(x) t_{a \beta}^{\alpha}\right) \delta^{4}(x-y) .
$$

Logo, podemos formalmente escrever o operador $\hat{D}$

$$
\hat{D}=-\left(\hat{P}^{2}-m^{2}+i \varepsilon\right)^{-1}
$$

por meio da representação do tempo próprio de Schwinger

$$
\hat{D}=i \int_{0}^{\infty} d \lambda e^{-i \hat{H}(\lambda)}, \hat{H}=-\lambda\left(\hat{P}^{2}-m^{2}+i \varepsilon\right) .
$$

O espaço $V$, que até agora permaneceu indeterminado, será definido como o setor de uma partícula do espaço de Fock dos operadores de criação e aniquilação $\hat{a}^{\dagger}$ e $\hat{a}$,

$$
\hat{a}_{\alpha}|0\rangle=0, \hat{a}_{\alpha}^{\dagger}|0\rangle=|\alpha\rangle,
$$

que satisfazem a álgebra

$$
\left[\hat{a}_{\alpha}^{\dagger}, \hat{a}_{\beta}\right]_{+}=\delta_{\alpha \beta},\left[\hat{a}_{\alpha}^{\dagger}, \hat{a}_{\beta}^{\dagger}\right]_{+}=\left[\hat{a}_{\alpha}, \hat{a}_{\beta}\right]_{+}=0 .
$$

Então é possível representar os operadores $\hat{t}_{a}$ como

$$
\hat{t}_{a}=\hat{a}_{\alpha}^{\dagger} t_{a \beta}^{\alpha} \hat{a}_{\beta}, t_{a \beta}^{\alpha}=\left\langle\alpha\left|\hat{t}_{a}\right| \beta\right\rangle .
$$

Aqui é imporante observar que os $\hat{t}_{a}$ satisfazem as relações de comutação da álgebra $s u(N)$,

$$
\left[\hat{t}_{a}, \hat{t}_{b}\right]=f_{a b}^{c} \hat{t}_{c}
$$

uma vez que os elementos de matriz $t_{a \beta}^{\alpha}$ satisfazem as relações de comutação (2.7) de $s u(N)$. Ademais, as propriedades de hermiticidade e de traço nulo de $t_{a \beta}^{\alpha}$ são herdadas pelos operadores $\hat{t}_{a}$,

$$
\begin{aligned}
& \operatorname{tr} \hat{t}_{a} \equiv \sum_{\alpha=1}^{M}\left\langle\alpha\left|\hat{t}_{a}\right| \alpha\right\rangle=t_{a \alpha}^{\alpha}=0 \\
& \hat{t}_{a}^{\dagger}=\left(\hat{a}_{\alpha}^{\dagger} t_{a \beta}^{\alpha} \hat{a}_{\beta}\right)^{\dagger}=\hat{a}_{\beta}^{\dagger} \bar{t}_{a \beta}^{\alpha} \hat{a}_{\alpha}=\hat{t}_{a},
\end{aligned}
$$


em que a involução $\dagger$ da álgebra abstrata de operadores conjuga os números complexos $t_{a \beta}^{\alpha}$. Finalmente, notamos que $\hat{t}_{a}$ conserva o número de partículas.

Usando a representação (2.10) para a base $t_{a}$, introduzimos estados coerentes fermiônicos $|\chi\rangle$ e $\langle\bar{\chi}|[87,48]$ definidos como a exponencial dos operadores de criação e aniquilação agindo no vácuo

$$
|\chi\rangle=D(\chi)|0\rangle,\langle\bar{\chi}|=| \chi\rangle^{\dagger}, D(\chi)=e^{\hat{a}^{\dagger} \chi-\hat{a} \bar{\chi}},\left[\hat{a}_{\alpha}, D(\chi)\right]_{+}=\chi_{\alpha} D(\chi),
$$

em que $\chi_{\alpha}$ e $\bar{\chi}_{\alpha}=\chi_{\alpha}^{\dagger}$ são números de Grassmann que comutam com o estado de vácuo e com $\hat{a}$ e $\hat{a}^{\dagger}$. Usando a relação $\hat{a}_{\alpha} D(\chi)=\chi_{\alpha} D(\chi)+D^{\dagger}(\chi) \hat{a}_{\alpha}$, fica evidente que os estados coerentes fermiônicos satisfazem

$$
\hat{a}^{\alpha}|\chi\rangle=\chi^{\alpha}|\chi\rangle,\langle\bar{\chi}| \hat{a}_{\alpha}^{\dagger}=\langle\bar{\chi}| \bar{\chi}_{\alpha} .
$$

A normalização desses estados segue da relação Baker-Campbell-Hausdorff,

$$
\langle\bar{\chi} \mid \xi\rangle=e^{\frac{1}{2}(\chi \bar{\chi}+\xi \bar{\xi}-2 \xi \bar{\chi})},
$$

e a resolução da indentidade é dada por

$$
\int \prod_{\alpha=1}^{N} d \bar{\chi}_{\alpha} d \chi^{\alpha}|\chi\rangle\langle\bar{\chi}|=\hat{1}, \int d \chi \chi=\int d \bar{\chi} \bar{\chi}=1 .
$$

Ela pode ser demonstrada facilmente, bastando notar que para um só componente, a expansão dá o operador identidade no espaço de Fock, $|0\rangle\left\langle 0\left|+a^{\dagger}\right| 0\right\rangle\langle 0| a$.

Por meio da resolução da identidade acima, torna-se possível relacionar elementos de matriz do setor de uma partícula do espaço de Fock na base $|\alpha\rangle$ à base de estados coerentes $|\chi\rangle$,

$$
\langle\alpha|\cdot| \beta\rangle=\int \prod_{\sigma, \kappa=1}^{N} d \bar{\chi}_{\sigma}^{\prime} d \chi^{\prime \sigma} d \bar{\chi}_{\kappa} d \chi^{\kappa} e^{\frac{1}{2}\left(\chi^{\prime} \bar{\chi}^{\prime}+\chi \bar{\chi}\right)} \chi^{\prime \alpha}\left\langle\bar{\chi}^{\prime}|\cdot| \chi\right\rangle \bar{\chi}_{\beta},
$$

onde foi usada a relação $\langle\bar{\chi} \mid \alpha\rangle=\bar{\chi}_{\alpha} \exp \frac{1}{2} \chi \bar{\chi}$. Como resultado, podemos reescrever o propagador (2.6) em termos de elementos de matriz de estados coerentes,

$$
D(x, y)_{\beta}^{\alpha}=\int \prod_{\sigma, \kappa=1}^{N} d \bar{\chi}_{\sigma}^{\prime} d \chi^{\prime \sigma} d \bar{\chi}_{\kappa} d \chi^{\kappa} e^{\frac{1}{2}\left(\chi^{\prime} \bar{\chi}^{\prime}+\chi \bar{\chi}\right)} \chi^{\prime \alpha}\left\langle x, \bar{\chi}^{\prime}|\hat{D}| y, \chi\right\rangle \bar{\chi}_{\beta}
$$

Na seção seguinte, os elementos de matriz $\left\langle x, \bar{\chi}^{\prime}|\hat{D}| y, \chi\right\rangle$ serão utilizados na obtenção de uma representação do propagador via integral de trajetória.

II. Uma outra interpretação possível do propagador $D(x, y)$ que aparece em (2.3) pode ser simplesmente como elementos de matriz

$$
D(x, y)^{i}{ }_{j}=\left\langle x\left|\hat{D}^{i}{ }_{j}\right| y\right\rangle
$$

dos vetores de base $|x\rangle$ do espaço de Hilbert abstrato $H$. O operador abstrato $\hat{D}$ adquire índices diretamente das matrizes da base de $s u(N)$. Note que renomeamos os índices da representação matricial de $s u(N)$. Os novos índices $i$ e $j$ denotam as componentes matriciais de uma nova base $T_{a}$,

$$
T_{a}=\frac{1}{4} \Gamma_{\alpha} t_{a \beta}^{\alpha} \Gamma_{\beta},\left[\Gamma_{\alpha}, \Gamma_{\beta}\right]=2 \delta_{\alpha \beta} .
$$


Esta base é bastante conveniente para obter representações por integral de trajetória do propagador por meio de técnicas adaptadas do caso da partícula espinorial. Entretanto, para que $T_{a}$ satisfaça às relações de comutação (2.1), é necessário que as matrizes $t_{a}$ sejam anti-simétricas, $t_{a}^{T}=-t_{a}$. Esta desvantagem pode ser contornada se tomarmos as matrizes $t_{a}$ na representação adjunta $t_{a b}^{c}=f_{a b}{ }^{c}$. Além desse caso, há outras representações em que é possível escolher as matrizes $t_{a}$ anti-simétricas. Por exemplo, no caso de $S U(2)$, é sempre possível escolher matrizes $t_{a}$ anti-simétricas para as representações de spin inteiro $s$. Nesse caso, $\alpha, \beta=1, \ldots, 2 s+1$ e $i, j=1, \ldots, 2^{s}$. No caso geral, na representação adjunta, $\alpha, \beta=1, \ldots, N^{2}-1$ e então $i, j=1, \ldots, 2^{\left[\left(N^{2}-1\right) / 2\right]}$. No caso familiar da representação adjunta de $s u(2)$, tem-se

$$
T_{a}=\frac{i}{4} \varepsilon_{a b c} \Gamma_{b} \Gamma_{c}=-\frac{i}{4} \varepsilon_{a b c} \Gamma_{b} \Gamma_{c}, a, b, c=1,2,3,
$$

em que as matrizes $\Gamma$ satisfazem $\left[\Gamma_{a}, \Gamma_{b}\right]_{+}=2 \delta_{a b}$ e são de ordem 2, então podem ser escolhidas como sendo as matrizes de Pauli, $\Gamma_{a}=\sigma_{a}^{2}$,

$$
T_{a}=-\frac{i}{4} \varepsilon_{a b c} \sigma_{b} \sigma_{c}=\frac{1}{2} \sigma_{a} .
$$

A base $T_{a}$ é composta de matrizes hermitianas e de traço nulo, e elas satisfazem as relações de comutação de $s u(2)$

$$
\left[T_{a}, T_{b}\right]=i \varepsilon_{a b c} T_{c} .
$$

Esse caso é especial, pois a escolha da representação adjunta para $t_{a}$ fornece $T_{a}$ na representação fundamental. Uma outra situação especial ocorre com $S U(4)$, em que se pode escolher $t_{a}$ como matrizes anti-simétricas de ordem 6 , uma vez que vale o isomorfismo de álgebras $s u(4) \simeq s o(6)$. Então, tem-se espinores pares ou ímpares de so (6) com 4 componentes, dando por meio do método descrito nas seções 3.1 e 4.1, a representação fundamental de $S U$ (4).

\subsection{Integral de trajetória na representação por estados coer- entes}

\subsubsection{Integral de trajetória}

Nosso objetivo nessa seção é escrever uma representação por integral de trajetória para

$$
D_{\chi}\left(x, \bar{\chi}^{\prime} ; y, \chi\right) \equiv\left\langle x, \bar{\chi}^{\prime} \mid \hat{D} y, \chi\right\rangle=i \int_{0}^{\infty} d \lambda\left\langle x, \bar{\chi}^{\prime}\left|e^{-i \hat{H}(\lambda)}\right| y, \chi\right\rangle
$$

Inserimos $N-1$ resoluções da identidade $I=\int d x d \bar{\chi} d \chi|x, \chi\rangle\langle x, \bar{\chi}|$ e $N$ integrações sobre $\lambda$ :

$$
\begin{aligned}
& D_{\chi}\left(x, \bar{\chi}^{\prime} ; y, \chi\right)=i \int_{0}^{\infty} d \lambda_{0} \int\left(\prod_{k=1}^{N-1} d x_{k} d \bar{\chi}_{k} d \chi_{k}\right) d \lambda_{1} \cdots d \lambda_{N} \\
& \prod_{k=1}^{N}\left\langle x_{k}, \bar{\chi}_{k}\left|e^{-i \hat{H}\left(\lambda_{k}\right) / N}\right| x_{k-1}, \chi_{k-1}\right\rangle \delta\left(\lambda_{k}-\lambda_{k-1}\right), \\
& { }^{2} \sigma_{1}=\left(\begin{array}{ll}
0 & 1 \\
1 & 0
\end{array}\right), \sigma_{2}=\left(\begin{array}{cc}
0 & -i \\
i & 0
\end{array}\right), \sigma_{3}=\left(\begin{array}{cc}
1 & 0 \\
0 & -1
\end{array}\right)
\end{aligned}
$$


em que $x_{N}=x, \bar{\chi}_{N}=\bar{\chi}^{\prime}, x_{0}=y$ e $\chi_{0}=\chi$. Com o intuito de calcular o elemento de matriz genérico que surge em (2.16), deve-se escolher uma prescrição de ordenamento para os operadores em $\hat{H}$. Em particular, deve-se resolver a ambiguidade no ordenamento do termo contendo quatro férmions em $\hat{P}^{2}$. Em [48], uma resolução adicional da identidade é inserida entre os operadores $\hat{P}$ como solução para o problema de ordenamento. Não sabemos a que prescrição esta solução corresponde, e prescrições convencionais tais como ordenamento de Weyl e ordenamento normal não são invariantes de calibre. No que segue, mostraremos que o ordenameno de Weyl não é invariante de calibre, e computaremos a ação efetiva resultante. Como é mostrado no apêndice (A.1), o operador Hamiltoniano difere da expressão obtida por ordenamento de Weyl ${ }^{3}$ pelo termo $\lambda \frac{q^{2}}{4} \operatorname{tr}\left(t_{a} t_{b}\right) \hat{A}_{\mu}^{a} \hat{A}^{\mu b}$. Esta ação, com exceção do termo não-invariante, é idêntica ao que se obteria duplicando a partição do tempo.

Aplicando a regra do ponto médio (A.3) ao elemento de matriz genérico, tem-se

$$
\begin{gathered}
\left\langle x_{k}, \bar{\chi}_{k}\left|\hat{H}\left(\lambda_{k}\right)\right| x_{k-1}, \chi_{k-1}\right\rangle=\int \frac{d p_{k}}{(2 \pi)^{4}} d \bar{\eta}_{k} d \eta_{k}\left\langle x_{k}, \bar{\chi}_{k} \mid p_{k}, \eta_{k}\right\rangle\left(H_{W}\left(\lambda_{k}\right)+Q\left(\lambda_{k}\right)\right)\left\langle p_{k}, \bar{\eta}_{k} \mid x_{k-1}, \chi_{k-1}\right\rangle, \\
H_{W}\left(\lambda_{k}\right) \equiv H_{W}\left(\lambda_{k}, \frac{x_{k}+x_{k-1}}{2}, p_{k}, \bar{\eta}_{k}, \frac{\eta_{k}+\chi_{k-1}}{2}\right) \\
Q\left(\lambda_{k}\right) \equiv \lambda_{k} \frac{q^{2}}{4} \operatorname{tr}\left(t_{a} t_{b}\right) A_{\mu}^{a}\left(\frac{x_{k}+x_{k-1}}{2}\right) A^{b \mu}\left(\frac{x_{k}+x_{k-1}}{2}\right),
\end{gathered}
$$

onde $H_{W}$ é o símbolo de Weyl de $\hat{H}_{W}$. Substituindo as funções delta $\delta\left(\lambda_{k}-\lambda_{k-1}\right)$ por suas representações integrais e usando a representação integral para a função delta fermiônica (A.5) nas integrações em $\chi$ e $\bar{\chi}$, temos

$$
\begin{aligned}
& D_{\chi}\left(x, \bar{\chi}^{\prime} ; y, \chi\right)=i \int_{0}^{\infty} d \lambda_{0} \int\left(\prod_{k=1}^{N-1} d x_{k}\right)\left(\prod_{k=1}^{N} \frac{d p_{k}}{(2 \pi)^{4}} d \lambda_{k} \frac{d \pi_{k}}{(2 \pi)} d \bar{\eta}_{k} d \eta_{k}\right) \exp \frac{1}{2}\left(\chi^{\prime} \bar{\chi}^{\prime}-\eta_{N} \bar{\eta}_{N}+2 \bar{\chi}^{\prime} \eta_{N}\right) \\
& \exp i \sum_{k=1}^{N}\left\{p_{k} \frac{\left(x_{k}-x_{k-1}\right)}{\Delta t}+\pi_{k} \frac{\left(\lambda_{k}-\lambda_{k-1}\right)}{\Delta t}-\frac{i}{2} \frac{\left(\eta_{k}-\eta_{k-1}\right)}{\Delta t} \bar{\eta}_{k}-\frac{i}{2} \frac{\left(\bar{\eta}_{k}-\bar{\eta}_{k-1}\right)}{\Delta t} \eta_{k-1}-H_{W}\left(\lambda_{k}\right)-Q\left(\lambda_{k}\right)\right\} \Delta t,
\end{aligned}
$$

onde $H_{W}\left(\lambda_{k}\right)=H_{W}\left(\lambda_{k}, \frac{x_{k}+x_{k-1}}{2}, p_{k}, \bar{\eta}_{k}, \frac{\eta_{k}+\eta_{k-1}}{2}\right), \eta_{0}=\chi$. O termo $\chi^{\prime} \bar{\chi}^{\prime}-\eta_{N} \bar{\eta}_{N}+2 \bar{\chi}^{\prime} \eta_{N}$ vem de $\left\langle\bar{\chi}^{\prime} \mid \eta_{N}\right\rangle$, e no limite $N \rightarrow \infty$ se reduz a $2 \bar{\chi}^{\prime} \eta(1)$. Tomando o limite $N \rightarrow \infty(\Delta t \rightarrow 0)$ e renomeando $\eta \rightarrow \chi$ e $\bar{\eta} \rightarrow \bar{\chi}$, tem-se

$$
\begin{aligned}
& D_{\chi}\left(x, \bar{\chi}^{\prime} ; y, \chi\right)=i \int_{0}^{\infty} d \lambda_{0} \int D x D p D \lambda D \pi D \bar{\chi} D \chi \exp i S_{e f f} \exp \bar{\chi}(1) \chi(1), \\
& S_{e f f}=\int_{0}^{1} d t\left(p \dot{x}+\pi \dot{\lambda}+\frac{i}{2}(\bar{\chi} \dot{\chi}-\dot{\bar{\chi}} \chi)+\lambda\left(\left(p_{\mu}+q A_{\mu}^{a} I_{a}\right)^{2}-m^{2}\right)-\frac{q^{2}}{4} \lambda \operatorname{tr}\left(t_{a} t_{b}\right) A_{\mu}^{a} A^{b \mu}\right),
\end{aligned}
$$

em que $I_{a}=\bar{\chi} t_{a} \chi$, e a integração funcional é feita sobre as trajetórias $x^{\mu}(t), p_{\mu}(t), \lambda(t), \pi(t)$, $\bar{\chi}(t)$ e $\chi(t)$, com valores de fronteira $x^{\mu}(0)=y^{\mu}, x^{\mu}(1)=x^{\mu}, \lambda(0)=\lambda_{0}, \bar{\chi}(1)=\bar{\chi}^{\prime}$ e $\chi(0)=\chi$.

\footnotetext{
${ }^{3}$ Ordenamento de Weyl aqui significa simetrização em todos os graus de liberdade bosônicos e anti-simetrização em todos os graus de liberdade fermiônicos.
} 
Como a integral de trajetória é invariante por translações, pode-se integrar sobre os momentos $p_{\mu}$ deslocando $p \mapsto p+\tilde{p}$, em que $\tilde{p}=-\dot{x} / 2 \lambda-q A^{a} I_{a}$ é a solução à equação clássica $\dot{x}=\partial H_{e f f} / \partial p$. Após a substituição $2 \lambda=e$, obtém-se a forma lagrangiana da integral de trajetória:

$$
\begin{gathered}
D_{\chi}\left(x, \bar{\chi}^{\prime} ; y, \chi\right)=i \int_{0}^{\infty} d \lambda_{0} \int D x D e D \pi D \bar{\chi} D \chi M[e, x] \exp i\left(S_{e f f}+S_{G}\right) \exp (\bar{\chi}(1) \chi(1)), \\
S_{\text {eff }}=\int_{0}^{1} d t\left(-\frac{\dot{x}^{2}}{2 e}-\frac{e}{2} m^{2}-q \dot{x}^{\mu} A_{\mu}^{a} I_{a}+\frac{i}{2}(\bar{\chi} \dot{\chi}-\dot{\bar{\chi}} \chi)\right)
\end{gathered}
$$

com medida lagrangiana e termo $S_{G}$ de fixação de calibre de parametrização

$$
\begin{aligned}
& M[e, x]=\int D p \exp \frac{i}{2} \int_{0}^{1} e\left(p^{2}-\frac{q^{2}}{4} \operatorname{tr} t_{a} t_{b} A_{\mu}^{a} A^{\mu b}\right) d t \\
& S_{G}=\int_{0}^{1} \pi \dot{e} d \tau
\end{aligned}
$$

Assim, a representação por integral de trajetória do propagador pôde ser deduzida a partir uma prescrição de ordenamento (ordenamento de Weyl) com o custo de uma medida de integração não-invariante de calibre.

\subsubsection{Ação pseudoclássica}

O funcional ação $S_{\text {eff }}$ em (2.18),

$$
S_{e f f}=\int_{0}^{1} d t\left(-\frac{\dot{x}^{2}}{2 e}-\frac{e}{2} m^{2}-q \dot{x}^{\mu} A_{\mu}^{a} I_{a}+\frac{i}{2}(\bar{\chi} \dot{\chi}-\dot{\bar{\chi}} \chi)\right), I_{a}=\bar{\chi} t_{a} \chi,
$$

é invariante por reparametrização,

$$
\delta_{\epsilon} S_{e f f}=0, \delta_{\epsilon} x=\epsilon \dot{x}, \delta_{\epsilon} e=\frac{d}{d t}(\epsilon e), \delta_{\epsilon} \chi=\epsilon \dot{\chi}, \delta_{\epsilon} \bar{\chi}=\epsilon \dot{\bar{\chi}} .
$$

No calibre $e=\sqrt{\dot{x}^{2}} / m$ coincide com a ação dada em [81, 43] que descreve uma partícula relativística escalar com coordenadas fermiônicas numa representação de um grupo de simetria $G$, cujas equações de movimento são

$$
m \frac{d}{d t} \frac{\dot{x}_{\mu}}{\sqrt{\dot{x}^{2}}}=q \dot{x}^{\nu} F_{\mu \nu}^{a} I_{a}, D_{t} \chi^{\alpha} \equiv \frac{d}{d t} \chi^{\alpha}+i q \dot{x}^{\mu} A_{\mu}^{a} t_{a \beta}^{\alpha} \chi^{\beta}=0
$$

onde $F_{\mu \nu}^{a}=\partial_{\mu} A_{\nu}^{a}-\partial_{\nu} A_{\mu}^{a}+i q f_{b c}^{a} A_{\mu}^{b} A_{\nu}^{c}$ é o tensor de campo e $D_{t}$ é a derivada covariante.

Com a finalidade de realizar análise canônica clássica ${ }^{4}$, é mais conveniente partir da ação invariante por reparametrização (2.21). Como esta ação não contem derivadas de $e$, é melhor considerar $e$ uma velocidade (veja [89]), e não introduzir seu momento conjugado. Chega-se assim ao seguinte hamiltoniano

$$
H=-\frac{e}{2} T-\dot{\chi}_{\alpha} \phi_{\alpha}-\dot{\bar{\chi}}_{\alpha} \bar{\phi}_{\alpha}
$$

\footnotetext{
${ }^{4}$ As definições e convenções são aquelas usadas em [88].
} 
onde o conjunto de vínculos $\Phi=\{T, \phi, \bar{\phi}\}$,

$$
T=\left(p_{\mu}+q A_{\mu}^{a} I_{a}\right)^{2}-m^{2}, \phi_{\alpha}=\pi_{\alpha}-\frac{i}{2} \bar{\chi}_{\alpha}, \bar{\phi}_{\alpha}=\bar{\pi}_{\alpha}-\frac{i}{2} \chi_{\alpha},
$$

define uma supermatriz degenerada $\{\Phi, \Phi\}$. A álgebra de vínculos se simplifica se considerarmos um conjunto equivalente de vínculos $\{\tilde{T}, \phi, \bar{\phi}\}$, onde $\tilde{T}$ é obtido de $T$ por meio dos deslocamentos $\chi \rightarrow \chi-i \bar{\phi}$ e $\bar{\chi} \rightarrow \bar{\chi}-i \phi$,

$$
\left\{\tilde{T}, \phi_{\alpha}\right\}=\left\{\tilde{T}, \bar{\phi}_{\alpha}\right\}=0,\left\{\phi_{\alpha}, \bar{\phi}_{\beta}\right\}=-i \delta_{\alpha \beta} .
$$

O novo hamiltoniano com multiplicadores de Lagrange redefinidos é

$$
\tilde{H}=\Lambda \tilde{T}+\Lambda_{\alpha} \phi_{\alpha}+\bar{\Lambda}_{\alpha} \bar{\phi}
$$

fornecendo a seguinte evolução temporal para os vínculos,

$$
\frac{d}{d t} \tilde{T}=0, \frac{d}{d t} \phi_{\alpha}=i \bar{\Lambda}_{\alpha}, \frac{d}{d t} \bar{\phi}_{\alpha}=i \Lambda_{\alpha},
$$

então a condição de conservação dos vínculos no tempo simplesmente fixam $\Lambda$ e $\bar{\Lambda}$. As equações de movimento para as variáveis independentes $\eta=\left(x^{\mu}, p_{\mu}, \chi_{\alpha}, \bar{\chi}_{\alpha}\right)$ são dadas por

$$
\dot{\eta}=\{\eta, \Lambda \tilde{T}\}_{D(\phi)}, \phi_{\alpha}=\bar{\phi}_{\alpha}=\tilde{T}=0,
$$

em que os parênteses de Dirac foram construídos com respeito ao conjunto de vínculos de segunda classe $\{\phi ; \bar{\phi}\}$. Usando propriedades bem conhecidas dos parênteses de Dirac, as equações de movimento se tornam

$$
\dot{\eta}=\{\eta, \Lambda T\}_{D(\phi)}, \phi_{\alpha}=\bar{\phi}_{\alpha}=T=0,
$$

E os parênteses não-nulos entre variáveis independentes são

$$
\left\{x^{\mu}, p_{\nu}\right\}_{D(\phi)}=\delta_{\nu}^{\mu},\left\{\chi_{\alpha}, \bar{\chi}_{\beta}\right\}_{D(\phi)}=-i \delta_{\alpha \beta} .
$$

Ademais, os $I_{a}$ são uma base covariantemente constante de $s u(N)$,

$$
\left\{I_{a}, I_{b}\right\}_{D(\phi)}=-i f_{a b}^{c} I_{c}, D_{t} I_{a} \equiv \frac{d}{d t} I_{a}+i q \dot{x}^{\mu} A_{\mu}^{b} f_{a b}^{c} I_{c}=0,
$$

que portanto serão conhecidos por isospin.

De (2.24), vemos que os operadores fermiônicos geram uma álgebra de operadores de criação e aniquilação

$$
\chi_{\alpha} \rightarrow a_{\alpha}, \bar{\chi}_{\alpha} \rightarrow a_{\alpha}^{\dagger},\left[a_{\alpha}, a_{\beta}^{\dagger}\right]_{+}=\delta_{\alpha \beta} .
$$

O espaço de Hilbert $\mathcal{H}$ pode ser realizado como produto direto do espaço de representação para a álgebra de Heisenberg e o espaço de Fock de $2^{n}$ dimensões dos operadores de criação e aniquilação

$$
\left|x ; \alpha_{1} \cdots \alpha_{p}\right\rangle=a_{\alpha_{1}}^{\dagger} \cdots a_{\alpha_{p}}^{\dagger}|x ; 0\rangle \in \mathcal{H}, p=0, . ., n .
$$


A ação do grupo $S O(2 n)$ preserva as relações de comutação (2.26), e os geradores de $s o(2 n)$ na representação acima são dados por $c_{\alpha \beta}=\left[a_{\alpha}^{\dagger}, a_{\beta}\right], a_{\alpha} a_{\beta}$ e $a_{\alpha}^{\dagger} a_{\beta}^{\dagger}$. Os $c_{\alpha \beta}$ pertencem à subálgebra $u(n)$ de $s o(2 n)$. Os $n$ operadores $c_{\alpha \beta}$ para $\alpha=\beta$ formam a subálgebra de $\operatorname{Cartan}^{5}$ de $s o(2 n)$.

A representação (2.27) é uma representação espinorial de dimensão $2^{n}$ de $s o(2 n)$, e suas representações irredutíveis são dadas pelos estados em que há um número par ou ímpar de operadores de criação, correspondendo à representação de Weyl (semi-espinorial) de $2^{n-1}$ dimensões de $s o(2 n)$. Esses estados podem ser decompostos ainda em representações irredutíveis de $s u(N)$, uma vez que os geradores de isospin $\hat{I}_{a}$ são uma combinação linear de geradores de $s o(2 n)$,

$$
\hat{I}_{a}=t_{a \alpha \beta} a_{\alpha}^{\dagger} a_{\beta}=\frac{1}{2} t_{a \alpha \beta}\left(\left[a_{\alpha}^{\dagger}, a_{\beta}\right]+\left[a_{\alpha}^{\dagger} a_{\beta}\right]_{+}\right)=\frac{1}{2} t_{a \alpha \beta} c_{\alpha \beta} .
$$

Logo, os $\hat{I}_{a}$ geram uma subálgebra $s u(N)$ de $s o(2 n)$.

Então, dada uma representação de $s u(N)$ em termos de matrizes $n \times n$, mostramos que a função de onda pertence a uma representação irredutível de $S O(2 n)$, i.e., uma representação de Weyl (semi-espinorial) de dimensão $2^{n-1}$. Para decompor essa representação em representações irredutíveis de $S U(N)$, é necessário calcular os autovalores de uma base da subálgebra de Cartan de $s u(N)$ na representação espinorial em questão. Sem perda de generalidade, assumimos que os $t_{a}$ pertencentes à base da subálgebra de Cartan de $s u(N)$ estejam diagonalizados. Com isto, (2.28) nos dá a decomposição dos geradores de isospin $\hat{I}_{a}$ numa combinação linear de elementos da base da subálgebra de Cartan de so $(2 n)$. A dimensão da subálgebra de Cartan de $s u(N)$ é $N-1$ : escolhemos $N-1$ elementos dentre os $N^{2}-1 \hat{I}_{a}$ e calculamos seus autovalores na representação semi-espinorial fixada. Isso nos dá as possíveis representações de $S U(N)$ contidas na função de onda.

Por exemplo, no caso de $S U(2)$, pode-se escolher a projeção de isospin $t_{3}$ e para $S U$ (3) pode-se usar a projeção de isospin $t_{3}$ e a hipercarga $Y$ como base para as subálgebras de Cartan respectivas, para caracterizar representações irredutíveis de $S U(N)$. Então esses geradores de isospin (já diagonalizados) são decompostos em termos dos $c_{\alpha}=\left[a_{\alpha}^{\dagger}, a_{\alpha}\right]$ (a base para subálgebra de Cartan de $s o(2 n)$ ) para obter seus autovalores para a representação espinorial de $s o(2 n)$ a qual pertence a função de onda. O intervalo de valores desses autovalores dá as representações irredutíveis de $S U(N)$.

No caso particular de $S U(2)$, a subálgebra de Cartan é gerada por um único elemento, i.e., $t_{3}$, cuja representação matricial numa base de autoestados de isospin $s$ (ou seja, numa representação de dimensão $n=2 s+1)$ é da forma $t_{3}=\operatorname{diag}(s, s-1, \ldots,-s+1,-s)$. A decomposição do operador de isospin $\hat{I}_{3}$ em geradores de Cartan de $s o(4 s+2)$ é da forma

$$
\hat{I}_{3}=\frac{s}{2} c_{1}+\frac{(s-1)}{2} c_{2}+\cdots+\frac{(-s)}{2} c_{2 s+1} .
$$

Cada $c_{\alpha}$ pode ter o valor mais ou menos um. Entretanto, a função de onda está em um estado com um número par de +1 (espinor de Weyl par) ou com um número ímpar de +1 (espinor de Weyl ímpar).

Por exemplo, para $s=1 / 2$,

$$
\hat{I}_{3}=\frac{1}{4} c_{1}-\frac{1}{4} c_{2}
$$

\footnotetext{
${ }^{5}$ A subálgebra de Cartan de uma álgebra compacta é a subálgebra abeliana maximal. A dimensão da subálgebra de Cartan de so $(2 n)$ é $n$, também conhecido como posto (rank).
} 
e portanto os autovalores possíveis de $\hat{t}_{3}$ para uma representação par $\left(c_{1}=c_{2}=+1\right.$ e $\left.c_{1}=c_{2}=-1\right)$ são duas vezes 0 , dando duas representações escalares; e para uma representação ímpar $\left(c_{1}=-c_{2}=\right.$ 1 e $\left.c_{1}=-c_{2}=-1\right)$ é $\pm 1 / 2$, dando a representação de isospin $1 / 2$. A função de onda pertence a uma representação semi-espinorial de $S O(4)$, portanto de dimensão 2.

Para cada spin inteiro, as representações pares ou ímpares se decompõem do mesmo modo, e a representação de isospin mais alto é $(s+1) s / 2$. Por exemplo, $s=1$ dá os autovalores $1,0,-1 \mathrm{e}$ novamente 0 , dando a representação de isospin 1 mais um escalar. Abaixo há uma tabela resumo dos resultados para alguns valores de isospin $(S U(2))$.

\begin{tabular}{|c|c|c|c|}
\hline isospin $s$ & grupo de simetria & dimensão da representação & decomposição(par;ímpar) \\
\hline \hline 0 & $S O(2)$ & 1 & $\underline{0}$ \\
\hline $1 / 2$ & $S O(4)$ & 2 & $2 \times \underline{0} ; \frac{1}{2}$ \\
\hline 1 & $S O(6)$ & 4 & $\underline{0}+\underline{1}$ \\
\hline $3 / 2$ & $S O(8)$ & 8 & $3 \times \underline{0}+\underline{2} ; 2 \times \frac{3}{2}$ \\
\hline 2 & $S O(10)$ & 16 & $\underline{0}+\underline{1}+\underline{2}+\underline{3}$ \\
\hline
\end{tabular}

Assim, para obter a representação fundamental de $S U(2)$ após a quantização, deve-se escolher o espaço de Hilbert como sendo a representação espinorial de Weyl ímpar de $S O$ (4) de espinores de duas componentes. Nesse caso, o vínculo $T$ dá a seguinte condição de quantização de Dirac

$$
\hat{T} \phi=\left[\left(\hat{p}_{\mu}+q A_{\mu}^{a} t_{a}\right)^{2}-m^{2}\right] \phi(x)=0
$$

que é precisamente a equação de onda em (2.3) para $t_{a}=\frac{1}{2} \sigma_{a}$.

Como último exemplo, considere o caso $S U(3)$ em que a base $t_{a}$ está na representação fundamental, i.e., são as matrizes de Gell-Man de ordem 3. A função de onda pertence a uma representação de espinorial de Weyl de $S O(6)$, de dimensão 4. $S U(3)$ possui posto dois, e portanto dois elementos da base podem ser simultaneamente diagonalizados, por exemplo, $t_{3}$ e a hipercarga $Y^{6}$

$$
t_{3}=\frac{1}{2}\left(\begin{array}{ccc}
1 & 0 & 0 \\
0 & -1 & 0 \\
0 & 0 & 0
\end{array}\right), Y=\frac{1}{3}\left(\begin{array}{ccc}
1 & 0 & 0 \\
0 & 1 & 0 \\
0 & 0 & -2
\end{array}\right) .
$$

Então, de acordo com (2.28),

$$
\hat{I}_{3}=\frac{1}{4}\left(c_{1}-c_{2}+0 \cdot c_{3}\right), \hat{Y}=\frac{1}{6}\left(c_{1}+c_{2}-2 c_{3}\right) .
$$

Se a função de onda é um espinor de Weyl par, então os autovalores possíveis de $\hat{I}_{3}$ são $0,1 / 2,-1 / 2$, 0 , e os autovalores possíveis $\hat{Y}$ são $0,-1 / 3,-1 / 3,2 / 3$, dando a representação anti-fundamental $\overline{3}$ de $S U$ (3) mais um singleto. Para função de onda um espinor de Weyl ímpar, obtém-se o estado fundamental $\underline{3}$ de $S U(3)$ mais um singleto.

Também é possível chegar a esses resultados a partir da ação clássica (2.21). No que segue, será conveniente expressar as variáveis de Grassmann $\chi$ em termos das suas partes reais e imaginárias,

$$
\begin{aligned}
& \chi_{\alpha}=\frac{1}{\sqrt{2}}\left(\chi_{1 \alpha}+i \chi_{2 \alpha}\right), \chi_{1 \alpha}^{\dagger}=\chi_{1 \alpha}, \chi_{2 \alpha}^{\dagger}=\chi_{2 \alpha}, \\
& \chi_{1 \alpha}=\frac{1}{\sqrt{2}}\left(\chi_{\alpha}+\bar{\chi}_{\alpha}\right), \chi_{2 \alpha}=\frac{1}{i \sqrt{2}}\left(\chi_{\alpha}-\bar{\chi}_{\alpha}\right) .
\end{aligned}
$$

\footnotetext{
${ }^{6}$ Aqui usamos as convenções de [90]
} 
Deste modo, a parte Grassmann do termo cinético fica

$$
L_{k i n}=\frac{i}{4}\left(\chi_{1} \dot{\chi}_{1}-\dot{\chi}_{1} \chi_{1}+\chi_{2} \dot{\chi}_{2}-\dot{\chi}_{2} \chi_{2}\right) \text {. }
$$

$L_{k i n}$ é invariante sob transformações induzidas por $R_{\alpha \beta}=-i\left(\chi_{1 \alpha} \chi_{1 \beta}+\chi_{2 \alpha} \chi_{2 \beta}\right)$ e $S_{\alpha \beta}=-i\left(\chi_{1 \alpha} \chi_{2 \beta}+\chi_{1 \beta} \chi_{2 \alpha}\right)$

$$
\begin{aligned}
& \delta_{\omega} \chi_{i \alpha} \equiv\left\{\frac{1}{2} \omega_{\beta \gamma} R_{\beta \gamma}, \chi_{i \alpha}\right\}_{D(\phi)}=\omega_{\alpha \beta} \chi_{i \beta}, \\
& \delta_{\lambda} \chi_{i \alpha} \equiv\left\{\frac{1}{2} \lambda_{\beta \gamma} S_{\beta \gamma}, \chi_{i \alpha}\right\}_{D(\phi)}=(-1)^{i+1} \lambda_{\alpha \beta} \chi_{i \beta}
\end{aligned}
$$

em que os parênteses de Dirac para as variáveis reais seguem dos parênteses das variáveis antigas (2.24) e de sua expressão em termos das variáveis reais (2.30),

$$
\left\{\chi_{1 \alpha}, \chi_{1 \beta}\right\}_{D(\phi)}=\left\{\chi_{2 \alpha}, \chi_{2 \beta}\right\}_{D(\phi)}=-i \delta_{\alpha \beta},\left\{\chi_{1 \alpha}, \chi_{2 \beta}\right\}_{D(\phi)}=0 .
$$

Os geradores de simetrias $R_{\alpha \beta}$ e $S_{\alpha \beta}$ satisfazem a álgebra de Lie

$$
\begin{aligned}
&\left\{R_{\alpha \beta}, R_{\gamma \delta}\right\}_{D(\phi)}=\delta_{\alpha \gamma} R_{\beta \delta}+\delta_{\beta \delta} R_{\alpha \gamma}-\delta_{\alpha \delta} R_{\beta \gamma}-\delta_{\beta \gamma} R_{\alpha \delta}, \\
&\left\{S_{\alpha \beta}, S_{\gamma \delta}\right\}_{D(\phi)}=\delta_{\alpha \gamma} R_{\beta \delta}+\delta_{\beta \delta} R_{\alpha \gamma}+\delta_{\alpha \delta} R_{\beta \gamma}+\delta_{\beta \gamma} R_{\alpha \delta}, \\
&\left\{R_{\alpha \beta}, S_{\gamma \delta}\right\}_{D(\phi)}=\delta_{\alpha \gamma} S_{\beta \delta}-\delta_{\beta \delta} S_{\alpha \gamma}+\delta_{\alpha \delta} S_{\beta \gamma}-\delta_{\beta \gamma} S_{\alpha \delta} .
\end{aligned}
$$

Acima reconhecemos as relações de comutação da seguinte combinação dos geradores $L_{i j}, i, j=$ $1, \ldots, 2 n$, de $o(2 n)$,

$$
R_{\alpha \beta}=L_{2 \alpha-1,2 \beta-1}+L_{2 \alpha, 2 \beta}, S_{\alpha \beta}=L_{2 \alpha, 2 \beta-1}-L_{2 \alpha-1,2 \beta}-\delta_{\alpha \beta} .
$$

Ademais, da decomposição dos geradores $I_{a}$ em termos das partes simétrica e anti-simétrica de $t_{a}$,

$$
\begin{aligned}
I_{a} & =t_{a(\alpha \beta)}\left(\bar{\chi}_{\alpha} \chi_{\beta}+\bar{\chi}_{\beta} \chi_{\alpha}\right)+t_{a[\alpha \beta]}\left(\bar{\chi}_{\alpha} \chi_{\beta}-\bar{\chi}_{\beta} \chi_{\alpha}\right) \\
& =\frac{i}{2} t_{a(\alpha \beta)}\left(\chi_{1 \alpha} \chi_{2 \beta}+\chi_{1 \beta} \chi_{2 \alpha}\right)+\frac{1}{2} t_{a[\alpha \beta]}\left(\chi_{1 \alpha} \chi_{1 \beta}+\chi_{2 \alpha} \chi_{2 \beta}\right), \\
& =-\frac{1}{2} t_{a(\alpha \beta)} S_{\alpha \beta}+\frac{i}{2} t_{a[\alpha \beta]} R_{\alpha \beta}
\end{aligned}
$$

nós novamente deduzimos que os $I_{a}$ são uma combinação linear dos geradores $R_{\alpha \beta}$ e $S_{\alpha \beta}$, que é o mesmo que dizer que os $I_{a}$ são os geradores da subálgebra $s u(N)$ de $s o(2 n)$. Trata-se de $s o(2 n)$ e não de $o(2 n)$, pois o traço de $S_{\alpha \beta}$ na expansão de $I_{a}$ não contribui, uma vez que as matrizes $t_{a}$ têm traço nulo.

\subsection{Integral de trajetória na representação da álgebra de Clif- ford}

\subsubsection{Integral de trajetória}

Aqui é usada a representação (2.13) para os geradores $T_{a}$ e técnicas [45, 91] empregadas no caso da partícula espinorial, adaptadas ao presente problema, para representar o propagador. Os índices 
$\alpha, \beta$ e $\gamma$ são os índices das matrizes $\Gamma$, isto é, são os índices do espaço de representação para a álgebra de Clifford. A representação do tempo próprio para o operador $\hat{D}(2.8)$ na representação da posição é

$$
D\left(x_{\text {out }}, x_{\text {in }}\right)=i \int_{0}^{\infty}\left\langle x_{\text {out }}\left|e^{-i \hat{H}(\lambda)}\right| x_{i n}\right\rangle d \lambda .
$$

A seguir é feita uma discretização por meio da inserção de $N-1$ resoluções da identidade $I=$ $\int d x|x\rangle\langle x|$ na expressão anterior,

$$
\begin{array}{r}
D\left(x_{\text {out }}, x_{i n}\right)=i \int_{0}^{\infty} d \lambda_{0} \int_{-\infty}^{\infty}\left(\prod_{i=1}^{N-1} d x_{i}\right) d \lambda_{1} \cdots d \lambda_{N} \\
\prod_{i=1}^{N}\left\langle x_{i}\left|e^{-i \hat{H}\left(\lambda_{i}\right) / N}\right| x_{i-1}\right\rangle \delta\left(\lambda_{i}-\lambda_{i-1}\right)
\end{array}
$$

em que $x_{N}=x_{\text {out }}$ e $x_{0}=x_{i n}$. Aplicando a correspondência simétrica ou de Weyl ao elemento de matriz geral, tem-se

$$
\left\langle x_{i}\left|e^{-i \hat{H}\left(\lambda_{i}\right) / N}\right| x_{i-1}\right\rangle=\int \frac{d p_{i}}{(2 \pi)^{4}} \exp \left(-\frac{i}{N} H\left(\lambda_{i}, \frac{x_{1}+x_{2}}{2}, p_{i}\right)\right) e^{i\left(x_{i}-x_{i-1}\right) p_{i}},
$$

em que $H$ é o símbolo de Weyl de $\hat{H}$,

$$
H(\lambda, x, p)=\lambda\left[m^{2}-\left(p_{i}^{2}+q p_{i}^{\mu} A_{\mu}^{a}(x) T_{a}\right)^{2}\right] .
$$

Como no caso da partícula espinorial [45], deve-se associar a cada matriz $T_{a}$ seu 'tempo' $\tau_{j}=j \Delta \tau$, tal que o propagador (2.33) ordenado no tempo se torna, para $1 / N \equiv \Delta \tau$,

$$
\begin{aligned}
D\left(x_{\text {out }}, x_{i n}\right) & =i T \int_{0}^{\infty} d \lambda \int_{-\infty}^{\infty}\left(\prod_{i=1}^{N-1} d x_{i}\right)\left(\prod_{i=1}^{N} \frac{\mathrm{d} p_{i}}{(2 \pi)^{4}} d \lambda_{i} \frac{d \pi_{i}}{2 \pi}\right) \\
& \times \exp i \sum_{i=1}^{N} S_{i}\left(x_{i}, x_{i-1}, p_{i}, \lambda_{i}, \pi_{i}\right)
\end{aligned}
$$

onde

$$
S_{i}=\left(\frac{x_{i}-x_{i-1}}{\Delta \tau} \cdot p_{i}-H\left(\lambda_{i}, \frac{x_{i}+x_{i-1}}{2}, p_{i}\right)+\pi_{i} \frac{\lambda_{i}-\lambda_{i-1}}{\Delta \tau}\right) \Delta \tau .
$$

No limite $\Delta \tau \rightarrow 0, S_{i} \rightarrow S_{H}\left[x, p ; \tau_{\text {in }}, \tau_{\text {out }}\right]$ é a ação Hamiltoniana, um funcional da trajetória $(x(t), p(t))$ no espaço de fase, no intervalo de tempo próprio $\left[\tau_{i n}, \tau_{o u t}\right]$, e $(2.35)$ é a versão discreta da seguinte integral de trajetória na forma Hamiltoniana

$$
D\left(x_{\text {out }}, x_{\text {in }}\right)=i T \int_{0}^{\infty} d \lambda_{0} \int_{x_{\text {in }}}^{x_{\text {out }}} D x \int D p \int_{\lambda_{0}} D \lambda D \pi \exp i \int_{\tau_{\text {in }}}^{\tau_{\text {out }}}(\dot{x} \cdot p-H(\lambda, x, p)+\pi \dot{\lambda}) d \tau .
$$


Seguindo [45], fontes ímpares $\rho_{a}(\tau)$ são introduzidas, anti-comutando com as matrizes $\Gamma$, e reescrevemos 2.37 como

$$
\begin{gathered}
D\left(x_{\text {out }}, x_{i n}\right)=i \int_{0}^{\infty} d \lambda_{0} \int_{x_{\text {in }}}^{x_{\text {out }}} D x \int D p \int_{\lambda_{0}} D \lambda D \pi \exp i \int_{0}^{1}\left[\lambda\left(\left(p_{\mu}+\frac{q}{4} t_{a \beta}^{\alpha} A_{\mu}^{a} \frac{\delta_{l}}{\delta \rho_{\alpha}} \frac{\delta_{l}}{\delta \rho_{\beta}}\right)^{2}-m^{2}\right)\right. \\
p \cdot \dot{x}+\pi \dot{\lambda} d \tau] \times\left. T \int_{0}^{1} \rho_{\alpha}(\tau) \Gamma^{\alpha} d \tau\right|_{\rho=0},
\end{gathered}
$$

em que por simplicidade fizemos $\tau_{\text {in }}=0$ e $\tau_{\text {out }}=1$. É possível apresentar o último termo do lado direito da equação acima como uma integral funcional [45, 91],

$$
\begin{aligned}
& T \int_{0}^{1} \rho_{\alpha}(\tau) \Gamma^{\alpha} d \tau=\exp \left(i \Gamma^{\alpha} \frac{\partial_{l}}{\partial \theta^{\alpha}}\right) \\
& \times\left.\int_{\psi(0)+\psi(1)=\theta} \exp \left[\int_{0}^{1}\left(\psi^{\alpha}(\tau) \dot{\psi}_{\alpha}(\tau)-i 2 \rho_{\alpha}(\tau) \psi^{\alpha}(\tau)\right) d \tau+\psi^{\alpha}(1) \psi_{\alpha}(0)\right] \mathcal{D} \psi\right|_{\theta=0} \\
& \mathcal{D} \psi=D \psi\left[\int_{\psi(0)+\psi(1)=0} \exp \int_{0}^{1} \psi^{\alpha}(\tau) \dot{\psi}_{\alpha}(\tau) d \tau\right]^{-1},
\end{aligned}
$$

em que $\theta$ são constantes ímpares, anti comutando com as matrizes $\Gamma$. Então, chegamos à representação por integral de trajetória hamiltoniana para o propagador:

$$
\begin{gathered}
D\left(x_{\text {out }}, x_{i n}\right)= \\
\quad i \exp \left(i \Gamma^{\alpha} \frac{\partial_{l}}{\partial \theta^{\alpha}}\right) \int_{0}^{\infty} d \lambda_{0} \int_{x_{\text {in }}}^{x_{\text {out }}} D x \int D p \int_{\lambda_{0}} D \lambda D \pi \\
\exp \left\{i \int _ { 0 } ^ { 1 } \left[\lambda\left(\left(p_{\mu}-q t_{a \beta}^{\alpha} A_{\mu}^{a} \psi_{\alpha} \psi_{\beta}\right)^{2}-m^{2}\right)\right.\right. \\
\left.\left.-i \psi^{\alpha} \dot{\psi}_{\alpha}+p \cdot \dot{x}+\pi \dot{\lambda}\right] d \tau+\psi^{\alpha}(1) \psi_{\alpha}(0)\right\}\left.\mathcal{D} \psi\right|_{\theta=0}, \\
x(0)=x_{i n}, x(1)=x_{\text {out }}, \lambda(0)=\lambda_{0}, \psi(0)+\psi(1)=\theta .
\end{gathered}
$$

Integrando sobre os momentos, encontra-se a representação lagrangiana da integral de trajetória:

$$
\begin{gathered}
D\left(x_{\text {out }}, x_{i n}\right)=\left.i \exp \left(i \Gamma^{\alpha} \frac{\partial_{l}}{\partial \theta^{\alpha}}\right) \int_{0}^{\infty} d e_{0} \int \exp \left\{i\left(S_{e f f}+S_{G}\right)+\psi^{\alpha}(1) \psi_{\alpha}(0)\right\} M[e, x] D x D e D \pi \mathcal{D} \psi\right|_{\theta=0} \\
S_{\text {eff }}=i \int_{0}^{1}\left(-\frac{\dot{x}^{2}}{2 e}-\frac{e}{2} m^{2}+q t_{a \beta}^{\alpha} \dot{x}^{\mu} A_{\mu}^{a} \psi_{\alpha} \psi_{\beta}-i \psi^{\alpha} \dot{\psi}_{\alpha}\right) \\
x(0)=x_{i n}, x(1)=x_{\text {out }}, e(0)=e_{0}, \psi(0)+\psi(1)=\theta
\end{gathered}
$$

em que a medida $M[e, x]$ e o termo de fixação de calibre de reparametrização $S_{G}$ são

$$
M[e, x]=\int D p \exp \frac{i}{2} \int_{0}^{1} e p^{2} d \tau, S_{G}=\int_{0}^{1} \pi \dot{e} .
$$




\subsubsection{Ação pseudoclássica}

Consideremos a ação invariante por reparametrização de (2.38) com a redefinição $\psi \rightarrow i / \sqrt{2} \psi$,

$$
S_{e f f}=\int d x^{4}\left(-\frac{\dot{x}^{2}}{2 e}-\frac{e}{2} m^{2}-q \dot{x}^{\mu} A_{\mu}^{a} I_{a}+\frac{i}{2} \psi^{\alpha} \dot{\psi}_{\alpha}\right), I_{a}=\frac{1}{2} t_{a \beta}^{\alpha} \psi_{\alpha} \psi_{\beta} .
$$

A ação acima é essencialmente aquela escrita em [81] no caso em que as variáveis de Grassmann $\psi$ pertencem à representação adjunta do grupo de Lie simples e compacto $G\left(t_{a \beta}^{\alpha}=f_{a c}^{b}\right)$, e em [43] para $\psi$ em uma representação com geradores anti-simétricos $t_{a}$. As equações de movimento no calibre $e=\sqrt{\dot{x}^{2}} / m$ são

$$
\begin{aligned}
& \frac{d}{d t}\left(m \frac{\dot{x}^{\mu}}{\sqrt{\dot{x}^{2}}}\right)=q F_{\mu \nu}^{a} \dot{x}^{\nu} I_{a}, D_{t} \psi^{\alpha} \equiv \frac{d}{d t} \psi^{\alpha}+i q \dot{x}^{\mu} A_{\mu}^{a} t_{a \beta}^{\alpha} \psi^{\beta}=0, \\
& F_{\mu \nu}^{a}=\partial_{\mu} A_{\nu}^{a}-\partial_{\nu} A_{\mu}^{a}+i q f_{b c}^{a} A_{\mu}^{b} A_{\nu}^{c},
\end{aligned}
$$

em que $F_{\mu \nu}^{a}$ é o tensor de campo não-abeliano.

A seguir, faremos uma análise canônica nos moldes daquela feita no caso da representação coerente, mas lembrando que as matrizes $t_{a}$ são anti-simétricas de ordem $n$. Como era esperado, a Hamiltoniana é proporcional aos vínculos,

$$
H=-\frac{e}{2} T-\dot{\psi}^{\alpha} \phi_{\alpha}
$$

onde

$$
T=\left(p_{\mu}+q A_{\mu}^{a} I_{a}\right)^{2}-m^{2}, \phi_{\alpha}=\pi_{\alpha}-\frac{i}{2} \psi_{\alpha}
$$

Depois de redefinir $T$ por meio do deslocamento $\psi \rightarrow \psi-i \phi, T \rightarrow \tilde{T}$, a álgebra de vínculos se torna

$$
\left\{\tilde{T}, \phi_{\alpha}\right\}=0,\left\{\phi_{\alpha}, \phi_{\beta}\right\}=-i \delta_{\alpha \beta} .
$$

O conjunto $\Phi=\{\tilde{T}, \phi\}$ é de primeira classe, e a evolução das variáveis independentes $\eta=(x, p, \psi)$ é

$$
\dot{\eta}=\{\eta, \Lambda T\}_{D(\phi)}=0, T=\phi_{\alpha}=0,
$$

em que os parênteses de Dirac são definidos com respeito ao conjunto de vínculos de segunda classe $\{\phi\}$. O comutador de Dirac das variáveis independentes é

$$
\left\{x^{\mu}, p_{\nu}\right\}_{D(\phi)}=\delta_{\nu}^{\mu},\left\{\psi_{\alpha}, \psi_{\beta}\right\}_{D(\phi)}=-i \delta_{\alpha \beta},
$$

As grandezas de isospin $I_{a}$ satisfazem a álgebra de Lie de $S U(N)$ após a quantização e são covariantemente constantes:

$$
\left\{I_{a}, I_{b}\right\}_{D(\phi)}=-i f_{a b}^{c} I_{c}, D_{\tau} I^{c}=\frac{d}{d \tau} I^{c}+i q \dot{x}^{\mu} A_{\mu}^{a} f_{a b}^{c} I^{b}=0 .
$$

É evidente que após a quantização as variáveis de Grassmann $\psi_{\alpha}$ geram uma álgebra de Clifford com $n$ geradores e um produto interno positivo definido. E então os estados físicos $\phi$ são vetores de $2^{[n / 2]}$ componentes satisfazendo

$$
\left[\left(\hat{p}_{\mu}+q A_{\mu}^{a} \hat{I}_{a}\right)^{2}-m^{2}\right] \phi(x)=0,
$$


em que os operadores de isospin quânticos $\hat{I}_{a}=\frac{1}{4} t_{a \beta}^{\alpha} \Gamma_{\alpha} \Gamma_{\beta}$ são precisamente aqueles introduzidos em (2.13) e satisfazem a álgebra de $s u(N)(2.1)$

$$
\left[\hat{I}_{a}, \hat{I}_{b}\right]=f_{a b}^{c} \hat{I}_{c}
$$

Façamos uma análise do conteúdo de isospin da teoria clássica na linha daquela que foi feita para o caso da representação por estados coerentes. Aqui, os termos cinéticos contendo variáveis de Grassmann na ação são invariantes por transformações geradas por $R_{\alpha \beta}=-i \psi_{\alpha} \psi_{\beta}$,

$$
\delta_{\omega} \psi_{\alpha}=\left\{\frac{1}{2} \omega_{\beta \gamma} R_{\beta \gamma}, \psi_{\alpha}\right\}_{D(\phi)}=\omega_{\alpha \beta} \psi_{\beta}, \omega_{\alpha \beta}=-\omega_{\beta \alpha},
$$

que fornecem uma representação para a álgebra de Lie $s o(n)$ :

$$
\left\{R_{\alpha \beta}, R_{\gamma \delta}\right\}_{D(\phi)}=\delta_{\alpha \gamma} R_{\beta \delta}-\delta_{\beta \gamma} R_{\alpha \delta}-\delta_{\alpha \delta} R_{\beta \gamma}+\delta_{\beta \delta} R_{\alpha \gamma} .
$$

Então os geradores $I_{a}$ são uma combinação linear de geradores $R_{\alpha \beta}$ de $s o(n)$, e portanto geram uma subálgebra $s u(N)$ de $s o(n)$. As representações espinoriais de $s o(n)$ são irredutíveis de dimensão $2^{(n-1) / 2}$ para $n$ ímpar e redutíveis de dimensão $2^{n / 2-1}$ para $n$ par.

A fim de determinar o conteúdo de $S U(N)$ da função de onda, procede-se como no caso anterior. Vimos que dados $t_{a}$ uma representação irredutível de $s u(N)$ em termos de matrizes $n \times n$ antisimétricas, a função de onda pertence a uma representação espinorial de $s o(n)$. Então são calculados os autovalores para uma base da subálgebra de Cartan de $s u(N)$ de acordo com a decomposição geral da base de isospin $I_{a}$ em termos da subálgebra de Cartan de $s o(n)$.

Por exemplo, no caso de $S U$ (2), a função de onda é um espinor de $2^{s}$ componentes de $S O(2 s+1)$, que possui uma subálgebra de Cartan de dimensão $s$. Logo, dada uma base no qual as matrizes $t_{a}$ são anti-simétricas (isso sempre é possível para spin inteiro), decompõe-se $I_{3}$ (o único elemento da base da subálgebra de Cartan de $s u(2))$ em elementos da base da subálgebra de Cartan de so $(2 s+1)$

$$
I_{3}=R_{23}+2 R_{45}+\cdots+s R_{2 s, 2 s+1} .
$$

Os autovalores possíveis dos elementos $R_{2 i, 2 i+1}, i=1, . ., s$ determinam por sua vez os autovalores possíveis de $I_{3}$, e portanto, as representações irredutíveis de $s u(N)$ aí contidas.

Abaixo há decomposições em $S U(2)$ da representação espinorial de $S O(2 s+1)$ para alguns valores de isospin:

\begin{tabular}{|c|c|c|c|}
\hline isospin $s$ & grupo de simetria & dimensão da representação & decomposição \\
\hline \hline 1 & $S O(3)$ & 2 & $\frac{1}{2}$ \\
\hline 2 & $S O(5)$ & 4 & $\underline{\frac{3}{2}}$ \\
\hline 3 & $S O(7)$ & 8 & $\underline{0}+\underline{3}$ \\
\hline
\end{tabular}

\subsection{Resumo}

Foram descritos dois métodos para obter ações clássicas de partículas escalares com isospin por meio de representações por integral de trajetória do propagador causal. A quantização de Dirac dessas ações produz as equações de onda correspondentes para várias representações de $S U(N)$. Por meio de uma escolha judiciosa da ação pseudoclássica e da representação da álgebra $s u(N)$ na ação, é possível obter a função de onda para o isospin desejado. 


\section{Capítulo 3}

\section{Estabilidade da teoria de gravitação de Jackiw-Teitelboim não-comutativa}

\subsection{Introdução}

De modo geral, a versão não-comutativa de uma teoria de campo é obtida pela substituição do produto ponto a ponto de funções pelo produto de Moyal (veja apêndice para definições). Esta extensão não é evidentemente única, mas em geral há restrições a serem obedecidas, como, por exemplo, a preservação do número de simetrias de calibre. No entanto, a análise da estrutura das simetrias de calibre em teorias não-comutativas é bastante complicada: por exemplo, uma interpretação de invariância de Lorentz conduz a uma simetria de Poincaré torcida [92].

Um passo importante em direção à construção de uma teoria de gravitação não-comutativa satisfatória foi recentemente dado por Wess e seus colaboradores [93], que entenderam como se deve construir invariantes por difeomorfismo, incluindo a ação de Einstein-Hilbert, em espaços nãocomutativos (veja também [94] para uma formulação real). Há, contudo, um preço a se pagar. O grupo de difeomorfismos se torna torcido, há um coproduto não-trivial devido à ação das simetrias em produtos tensoriais que tem uma aparência bem pouco familiar [95, 96].

Em duas dimensões é possível construir modelos não-comutativos de gravitação dilatônica com uma realização usual (não-torcida) das simetrias de calibre. Uma versão não-comutativa do modelo de Jackiw-Teitelboim (NCJT) foi construída em [97] e então quantizada em [98]. Um buraco negro de Witten não-comutativo foi sugerido em [99]. Ambos modelos são do tipo Yang-Mills: o modelo de JT é equivalente a um modelo BF topológico; o buraco negro de Witten pode ser representado por um modelo de Wess-Zumino-Novikov-Witten. Há procedimentos gerais de como tais modelos possam ser formulados no caso não-comutativo (veja [97, 100]). É importante, portanto, verificar se se pode ir além do paradigma de Yang-Mills. Além do mais, um indicativo de que este é o caminho correto seria a constatação de que teorias de gravitação dilatônicas existiriam não só para potenciais constantes ou lineares, mas para potenciais arbitrários. Nós verificaremos aqui se potenciais quadráticos são permitidos.

A fim de analisar as simetrias de calibre, nós usamos o formalismo canônico para espaços-tempo

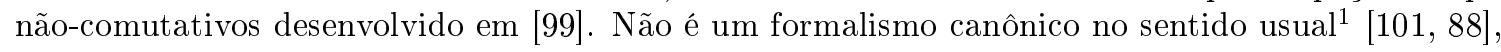

\footnotetext{
${ }^{1}$ Uma vez que teorias em espaços-tempo não-comutativos não são locais no tempo e contêm um número infinito
} 
mas torna possível que se defina a noção de vínculos de primeira classe e se associe uma simetria de calibre a eles, ao menos para a classe de modelos que podem ser postos na forma

$$
S=\int\left(p_{a} \partial_{0} q^{a}-h(p, q, \lambda)\right) d^{D} x
$$

em que $h$ é uma função-^ local ${ }^{2}$. A ação acima permite a identificação de pares canonicamente conjugados, isto é, $q_{a}$ e $p^{a}$ desempenharão o papel de coordenadas e momentos, respectivamente, e definirão uma estrutura de Poisson primitiva, por meio da aplicação bilinear anti-simétrica $\left(q^{a}, p_{b}\right)=$ $-\left(p_{b}, q^{a}\right)=\delta_{b}^{a}$. Finalmente, $\lambda$ são multiplicadores de Lagrange.

No caso de teorias de calibre comutativas, há uma conjectura feita por Dirac de que os vínculos de primeira classe são os geradores das transformações de calibre. Para certas classes de teorias de calibre comutativas, isto pode ser provado e, também, verifica-se que o número de transformações de calibre independentes é igual ao número de vínculos de primeira classe primários [88]. A estrutura de simetria de uma teoria de calibre comutativa geral foi recentemente descrita em detalhe e relacionada à estrutura de vínculos na formulação hamiltoniana [102]. Em particular, a carga de calibre foi construída explicitamente como uma decomposição numa base ortogonal de vínculos. Demonstrouse que, no caso geral, a carga de calibre não pode ser construída com vínculos de primeira classe apenas, pois sua decomposição também envolve combinações especiais de vínculos de segunda classe.

De acordo com essa ideologia, introduz-se o parêntese canônico no espaço dos funcionais-^ locais $^{3}$, uma aplicação bilinear daquele espaço nele mesmo, anti-simétrica, e que satisfaz a identidade de Jacobi. Se denotarmos por $\eta_{i}=\left(q^{a}, p_{b}\right)$ as variáveis canônicas, e por $F$ um monômio genérico de um funcional-^ local, isto é,

$$
F=\int d^{D} x \partial_{\mu_{1}} \eta_{1} \star \partial_{\mu_{2}} \eta_{2} \star \cdots \partial_{\mu_{n}} \eta_{n},
$$

em que $\mu_{i}$ é um multi-índice e $\partial_{\mu_{i}}$ é um operador diferencial de ordem $\left|\mu_{i}\right|$, o parêntese canônico é dado por

$$
\begin{aligned}
\left\{F, F^{\prime}\right\}= & \sum_{i, j} \int d^{D} x \partial_{\mu_{j}}\left(\partial_{\mu_{j+1}} \eta_{j+1} \star \cdots \star \partial_{\mu_{j-1}} \eta_{j-1}\right)\left(\eta_{j}, \eta_{i}^{\prime}\right) \\
& \star \partial_{\mu_{i}^{\prime}}\left(\partial_{\mu_{i+1}^{\prime}} \eta_{i+1}^{\prime} \star \cdots \star \partial_{\mu_{i-1}^{\prime}} \eta_{i-1}^{\prime}\right)(-1)^{\left|\mu_{j}\right|+\left|\mu_{i}^{\prime}\right|} .
\end{aligned}
$$

O produto acima é calculado do seguinte modo: 1)para cada par de variáveis canonicamente conjugadas $\left(\eta_{j}, \eta_{i}^{\prime}\right)$, permuta-se ciclicamente em $F$ o elemento $\eta_{j}$ até ele ocupar o último lugar à direita, e analogamente, permuta-se ciclicamente em $F^{\prime}$ o elemento $\eta_{i}^{\prime}$ até ele ocupar o primeiro lugar à esquerda; 2)então integra-se por partes cada uma das expressões $F$ e $F^{\prime}$ para remover as derivadas de $\eta_{j}$ e $\eta_{i}^{\prime}$; 3)depois, eliminam-se $\eta_{j}$ e $\eta_{i}^{\prime}$ e multiplicam-se com o produto de Moyal os integrandos resultantes de $F$ e $F^{\prime}$, e ao resultado acrescenta-se o fator $\left(\eta_{i}, \eta_{j}^{\prime}\right)$; e 4)finalmente, integra-se sobre $\mathcal{M}$.

Em particular, $p^{i}$ se tornam canonicamente conjugados aos $q_{i}$,

$$
\left\{q_{a}(x), p^{b}(y)\right\}=\delta_{a}^{b} \delta^{2}(x-y) .
$$

de derivadas temporais no produto estrela, é evidente que alguma modificação do formalismo canônico padrão seja necessário.

${ }^{2}$ Uma função-^ local é um elemento do fecho do conjunto dos polinômios das variáveis canônicas e suas derivadas calculados com o produto de Moyal.

${ }^{3}$ Funcionais- $\star$ locais são integrais em $\mathcal{M}$ das funções- $\star$ locais. 
A propriedade mais incomum do colchete (3.3) é a presença da função delta das coordenadas temporais no lado direito. Entretanto, como teorias em espaços-tempo não-comutativos não são locais no tempo, a restrição dos parênteses entre variáveis do espaço de fase no mesmo instante de tempo não parece natural ou mesmo consistente. A presença de um função delta a mais em (3.3) lembra o formalismo de Ostrogradski para teorias com derivadas temporais elevadas (veja [103, 104, 105, 106] e [99] para uma discussão mais extensa). De todo modo, também se podem utilizar os parênteses (3.3) para analisar simetrias de calibre em teorias comutativas. Não é claro, entretanto, se se pode utilizar esses parênteses modificados para fins de quantização. Aqui usaremos exclusivamente (3.3) para definir a estrutura de Poisson.

Nós iremos demonstrar que não é possível somar consistentemente termos quadráticos ao potencial dilatônico do modelo NCJT, portanto ele é estável ante tais deformações.

\subsubsection{Gravitação em 2 e 3 dimensões}

Gravitação em 2 e 3 dimensões [107] é um laboratório de estudo de questões pouco compreendidas em 4 dimensões, analogamente ao que é feito em dimensões baixas para o estudo de outros fenômenos, tais como quebra espontânea de simetria, anomalias, confinamento, sólitons, etc.

No entanto, gravitação em dimensões baixas é bastante peculiar. Para começar, em 3 dimensões, a equação de Einstein $G_{\mu \nu}+\Lambda g_{\mu \nu}=\kappa T_{\mu \nu}$ pode ser escrita como

$$
R_{\mu \nu}=2 \Lambda g_{\mu \nu}+\kappa\left(T_{\mu \nu}-g_{\mu \nu} T_{\sigma}^{\sigma}\right) .
$$

E ademais, levando em conta as simetrias do tensor de curvatura, constata-se que ele só possui 6 componentes independentes, i.e., em número igual ao do tensor de Ricci $R_{\mu \nu}$. Ou, seja, o tensor de curvatura é completamente determinado pelo tensor de Ricci:

$$
R_{\mu \nu \lambda \rho}=g_{\mu \lambda} R_{\nu \rho}+g_{\nu \rho} R_{\mu \lambda}-g_{\mu \rho} R_{\nu \lambda}-g_{\nu \lambda} R_{\mu \rho}-\frac{1}{2} R\left(g_{\mu \lambda} g_{\nu \rho}-g_{\mu \rho} g_{\nu \lambda}\right)
$$

e pela expressão anterior, isto implica que o tensor de curvatura é completamente fixado pela distribuição local de matéria $T_{\mu \nu}$ e pela constante de cosmológica $\Lambda$. Em particular, as regiões livres de fontes, $T_{\mu \nu}=0$, são regiões de curvatura constante, onde o tensor de curvatura vale

$$
R_{\mu \nu \lambda \rho}=\Lambda\left(g_{\mu \lambda} g_{\nu \rho}-g_{\mu \rho} g_{\nu \lambda}\right)
$$

e a curvatura escalar $R=6 \Lambda$. Isto quer dizer que efeitos de curvatura produzidos pela matéria não se propagam pelo espaço-tempo: não há graus de liberdade dinâmicos. Obs: Não obstante a curvatura local nas regiões livres de matéria não seja afetada pelas mesmas, a matéria pode produzir efeitos globais de curvatura não-triviais.

Em 2 dimensões o tensor de curvatura só tem um componente independente, uma vez que todos os componentes não nulos podem ser obtidos a partir de $R_{0101}$ usando as simetrias do tensor. Isto implica que o tensor de curvatura pode ser escrito em termos do escalar de curvatura $R$,

$$
R_{\mu \nu \lambda \rho}=\frac{1}{2} R\left(g_{\mu \lambda} g_{\nu \rho}-g_{\mu \rho} g_{\nu \lambda}\right) .
$$

Esta relação entre o tensor de curvatura e o escalar de curvatura resulta no fato de que o tensor de Einstein $G_{\mu \nu}$ é identicamente nulo em 2 dimensões:

$$
R_{\mu \nu}-\frac{1}{2} g_{\mu \nu} R \equiv 0
$$


Além disso, pode-ser constatar a partir da expressão da curvatura escalar em termos das formas de conexão, $R=2 \epsilon^{\mu \nu} \partial_{\mu} \omega_{\nu}$, que a ação de Einstein-Hilbert contem uma divergência total. Essa integral, para uma variedade Riemanniana compacta $M$ de genus $\gamma$, é a característica de Euler

$$
\int_{M} d^{2} x \sqrt{g} R=8 \pi(1-\gamma) .
$$

Então, uma nova teoria clássica se faz necessária em 2 dimensões, e um bom candidato para uma equação de campo gravitacional de segunda ordem no vácuo parece ser

$$
R+\Lambda=0,
$$

já que todo conteúdo de curvatura se encontra no escalar de Ricci. Esta equação foi primeiro sugerida por Jackiw e Teitelboim em [108].

\subsubsection{Modelo de Jackiw-Teitelboim}

Evidentemente, a ação

$$
\int_{M} d^{2} x \sqrt{-g}(R+\Lambda)
$$

não é útil para fornecer as novas equações de movimento, pois, como se viu, o termo com curvatura é uma divergência total, restando apenas a equação $\Lambda=0$, e portanto a métrica permanece indefinida. Por outro lado, não há ação invariante construída somente a partir da métrica e que seja a integral no tempo de uma lagrangiana local que dê a equação (3.4). A saída que Jackiw e Teitelboim [108] encontraram para contornar esta limitação foi a introdução de um campo escalar auxiliar $\phi$ (o dílaton):

$$
S=\int_{M} d^{2} x \sqrt{-g} \phi(R+\Lambda) .
$$

Variação desta ação com respeito ao campo $\phi$ dá a equação de Einstein bidimensional (3.4), e variação com respeito à metrica dá a seguinte equação para o campo $\phi$ :

$$
\left(\nabla_{\mu} \nabla^{\mu}-\Lambda\right) \phi=0 .
$$

Mais tarde $([109,110])$, percebeu-se que estas equações de movimento poderíam ser formuladas no contexto de uma teoria topológica de calibre com simetria do grupo de de Sitter $S O(2,1)$. Uma constante cosmológica não-nula se faz necessária, porque o grupo de Poincaré em duas dimensões, $I S O(1,1)$, não possui uma forma quadrática bilinear simétrica não-degenerada (forma de Killing), essencial para definição da métrica no espaço-tempo. Então, a álgebra do grupo de Poincaré bidimensional é deformada para a álgebra de de Sitter

$$
\left[\tau_{i}, \tau_{j}\right]=-\epsilon_{i j}^{k} \tau_{k}, i, j, k=0,1,2,
$$

com a introdução de uma constante cosmológica. A métrica de Killing de so $(2,1)$,

$$
\eta_{i j}=\frac{1}{2} \epsilon_{i k}^{l} \epsilon_{j}^{k}=\operatorname{diag}(-1,1,1)
$$

pode ser usada para normalizar a base $\tau_{i}$ de $s o(2,1)$ de acordo com $\operatorname{tr} \tau_{i} \tau_{j}=\frac{1}{2} \eta_{i j}$. Já a conexão de calibre $A$ pode ser escrita como um multipleto composto pelos referenciais móveis (zweibeine) $e^{a}$, 
$a=0,1$, e pela conexão de spin $\omega, A=e^{a} \tau_{a}+\omega \tau_{2}$. Com isto, a métrica no espaço-tempo passa a ser escrita em termos dos referenciais móveis

$$
g\left(e^{a}, e^{b}\right)=\eta^{a b} \Longrightarrow g_{\mu \nu}=\eta^{a b} e_{\mu}^{a} e_{\nu}^{b} .
$$

O tensor de campo $F$ é uma 2-forma que toma valores na álgebra de Lie so $(2,1)$, dado pela expressão ${ }^{4}$

$$
F=d A+\frac{1}{2}[A, A] \equiv d A+A^{i} \wedge A^{j}\left[\tau_{i}, \tau_{j}\right] .
$$

Note que em duas dimensões a identidade de Bianchi $D F=0$ é trivialmente satisfeita, pois $D F$ é uma 3 -forma. Em componentes da base $\tau_{i}$, o tensor de campo se decompõe na torsão 1 -forma para $A=0,1$,

$$
F^{a} \equiv T^{a}=d e^{a}+\omega^{a}{ }_{b} \wedge e^{b}, \omega^{a}{ }_{b}=\varepsilon^{a}{ }_{b} \omega,
$$

e para $A=2$ em um termo dual à curvatura escalar $R=2 * d \omega$ e um termo de volume,

$$
F^{2}=d \omega+\frac{1}{2} \varepsilon_{a b} e^{a} \wedge e^{b}
$$

Por uma transformação conforme com parâmetro constante, $e^{a} \mapsto \sqrt{\Lambda / 2} e^{a}$, temos que $e R \mapsto e R$ quando a torsão é nula, $F^{a}=0$, e portanto a equação $F^{2}=0$ é equivalente a

$$
e(R+\Lambda)=0 .
$$

Logo, a equação de Einstein em duas dimensões é equivalente a $F^{A}=0$. A maneira mais direta de obter essa equação de movimento a partir do princípio de mínima ação, sem comprometer a invariância da ação por transformações de calibre, é integrar sobre a 2-forma $\Phi F$, em que $\Phi$ é um multipleto escalar que se transforma na representação adjunta de $s o(2,1)$,

$$
S=\int_{M} \operatorname{tr}(\Phi F)
$$

A ação acima é invariante por transformações infinitesimais geradas por $\lambda=\lambda^{A} \tau_{A}$

$$
\delta_{\lambda} A=D \lambda=d \lambda+[A, \lambda], \delta_{\lambda} \Phi=[\Phi, \lambda] .
$$

Não é difícil ver que difeomorfismos $\delta_{\varepsilon} x^{\mu}=\varepsilon^{\mu}(x)$ estão contidos 'on-shell' nas transformações acima, pois são gerados por transformações infinitesimais com parâmetro $\lambda=\varepsilon^{\mu} A_{\mu}$ quando $F^{A}=0$.

A equação de movimento para o campo escalar $\Phi$,

$$
D \Phi=d \Phi+[A, \Phi]=0,
$$

é equivalente à equação (3.6), se fizermos a decomposição do multipleto em $\Phi=\left(\phi^{a}, \phi\right)$ e usarmos a equação para $\phi^{a}$ para eliminarmos essas componentes, resultando na equação de Klein-Gordon no espaço de de Sitter,

$$
\left(\nabla_{\mu} \nabla^{\mu}+\Lambda\right) \phi=0
$$

\footnotetext{
${ }^{4}$ Veja a fórmula (B.2) do apêndice para definição do comutador.
} 


\subsubsection{Gravitação dilatônica}

Os modelos de gravitação dilatônica em duas dimensões [111] podem ser genericamente agrupadas na ação

$$
S=\int_{M} d^{2} x \sqrt{-g}\left[\frac{R}{2} \phi-\frac{U(\phi)}{2}(\nabla \phi)^{2}+V(\phi)\right]
$$

ou, no formalismo de primeira ordem,

$$
S=\int_{M}\left[\phi_{a} D e^{a}+\phi d \omega+\epsilon\left(\frac{\phi_{a} \phi^{a}}{2} U(\phi)+V(\phi)\right)\right]
$$

que corresponde a uma generalização do modelo de Jackiw e Teitelboim e se reduzem a esse com a escolha $U=0$ e $V=\Lambda \phi[?]$.

Há outros exemplos interessantes que conduzem a modelos de gravitação dilatônica, como:

- Uma variedade $M_{D}$ em $D$ dimensões com simetria esférica pode ser decomposta no produto $M_{D}=M \otimes S_{D-2}$

$$
d s^{2}=g_{\mu \nu}(x) x^{\mu} x^{\nu}-\lambda^{-2} \phi^{2 / D-2}(d \Omega)_{S_{D-2}}^{2},
$$

tal que a ação de Einstein-Hilbert se reduza a

$$
S^{E H}=\frac{A_{D-2}}{\lambda^{D-2} 16 \pi G} \int_{M} d^{2} x \sqrt{-g}\left[\phi R+\frac{D-3}{D-2} \frac{(\nabla \phi)^{2}}{\phi}-\lambda^{2}(D-2)(D-3) \phi^{(D-4) / D-2}\right],
$$

um caso particular de gravitação dilatônica. Esse caso corresponde à escolha $V \propto \phi^{\frac{D-4}{D-2}}$ e $U \propto \phi^{-1}$.

- Gravitação de cordas ou buraco negro de Witten $[112,113,114]$ : esse caso corresponde à escolha $V=-2 \lambda^{2} \phi$ e $U=-\phi^{-1}$.

O interesse pela gravitação dilatônica em duas dimensões também se deve ao fato de funcionarem como "toy models" para o estudo da quantização da gravidade e da evaporação de buracos negros, e por possuir conexões com geometria não-comutativa e quantização por deformação.

Por último, entre os vários contextos em que esses modelos fazem contacto com a teoria de cordas, um caso interessante é que as condições de invariância conforme para a a ação de modelo sigma não-linear da corda bosônica

$$
L^{(\text {sig })}=\frac{1}{4 \pi \alpha^{\prime}} \int d^{2} \sigma \sqrt{-h}\left[h^{\alpha \beta} \partial_{\alpha} X^{\mu} \partial_{\beta} X_{\mu}+\alpha^{\prime} \Phi \mathcal{R}\right]
$$

são equivalentes às equações de movimento para a seguinte ação de gravitação dilatônica (veja [115])

$$
L^{(d i l)}=\int d^{D} X \sqrt{-g} e^{-2 \Phi}\left[R+4(\nabla \Phi)^{2}-4 \lambda^{2}\right] .
$$

A partir de uma transformação conforme $g \rightarrow e^{2 \rho} g \operatorname{com} \rho=1 / 2 \int^{\phi} U(y) d y$, a ação (3.7) passa a descrever teorias de gravitação dilatônica com $\tilde{U}=0$ e $\tilde{V}=e^{-2 \rho} V$. Esta simplificação da descrição de teorias de gravitação dilatônica em duas dimensões em termos de um único potencial acontece às custas da restrição da função $U$ da formulação original a uma função não-singular, tal que as 
duas formulações sejam fisicamente equivalentes. Com esta restrição em mente, vamos utilizar a seguinte forma geral para teorias de gravitação dilatônica em duas dimensões,

$$
S=\int d^{2} x \varepsilon^{\mu \nu}\left(\phi \partial_{\mu} \omega_{\nu}+\phi_{a} D_{\mu} e_{\nu}^{a}-\varepsilon_{a b} e_{\mu}^{a} e_{\nu}^{a} V(\phi)\right),
$$

em que $e_{\mu}^{a}$ é o zweibein, $\varepsilon^{\mu \nu}$ é o símbolo de Levi-Civita (veja o apêndice para nossas convenções de sinal). A derivada covariante

$$
\varepsilon^{\mu \nu} D_{\mu} e_{\nu}^{a}=\varepsilon^{\mu \nu}\left(\partial_{\mu} e_{\nu}^{a}+\omega_{\mu} \varepsilon_{b}^{a} e_{\nu}^{b}\right)
$$

contem a conexão de spin $\omega_{\mu} \varepsilon_{b}^{a}$. Aqui $\phi$ é um campo escalar conhecido por dílaton. $\phi_{a}$ é um campo auxiliar. No caso comutativo, que estamos considerando no momento, qualquer escolha do potencial $V(\phi)$ leva a um modelo consistente.

O campo auxiliar $\phi_{a}$ gera a condição de que $\omega_{\mu}$ é a conexão de Levi-Civita compatível com a métrica. Sob essa condição $\varepsilon^{\mu \nu} \partial_{\mu} \omega_{\nu}$ se torna proporcional ao tensor de curvatura de Riemann (os termos proporcionais a $\phi_{a}$, naturalmente, desaparecem). Desse modo, chega-se ao formalismo de segunda ordem. Entretanto, a ação de primeira ordem (3.8) tem muitas vantagens sobre a de segunda ordem. Por exemplo, as equações clássicas de movimento são muito mais fáceis de resolver [116], e no caso quântico, é possível realizar uma integral de trajetória sobre as variáveis geométricas mesmo na presença de campos de matéria adicionais [117, 118, 119].

\subsection{Gravitação não-comutativa de Jackiw-Teitelboim}

Uma versão não-comutativa do modelo de Jackiw-Teitelboim foi construída em [97]. Essa versão foi identificada com uma teoria de calibre $U(1,1)$ definida numa variedade $M$ bidimensional,

$$
S_{N C J T}=\int_{M} \operatorname{Tr} \Phi \star F,
$$

em que $\Phi$ é uma 0 -forma com valores na álgebra de Lie $u(1,1)$ e $F$ é uma 2 -forma com valores na álgebra de Lie $u(1,1)$, i.e., $F$ é o tensor de campo de Yang-Mills ${ }^{5} F=d A+\frac{1}{2}[A, A]$.

A ação (3.10) é invariante por transformações de calibre infinitesimais geradas por $\lambda=\lambda^{A} \tau_{A}$ :

$$
\delta_{\lambda} A=D \lambda, \delta_{\lambda} \Phi=[\lambda, \Phi] .
$$

Assim como no caso comutativo, as equações de movimento implicam que $\Phi$ é covariantemente constante e que a conexão $A$ é plana:

$$
F=0, D \Phi=0,
$$

ou seja, não há graus de liberdade se propagando; a teoria é topológica.

Escolhendo uma base para $u(1,1)$ dada pelas matrizes $\tau_{A}, A=0,1,2,3$, do apêndice, a ação $S_{N C J T}$ se reescreve como

$$
S_{N C J T}=\frac{1}{2} \int_{M} \eta_{A B} \Phi^{A} \star F^{B} .
$$

\footnotetext{
${ }^{5}$ Veja fórmula (B.3) do apêndice para definição do comutador no contexto não-comutativo.
} 
De acordo com a decomposição $\Phi^{A}=\left(l \phi^{a}, \phi, \psi\right)$ e $A^{A}=\left(l^{-1} e^{a}, \omega, b\right)$, e levando em conta o produto entre os elementos da base, temos

$$
F=d A+A^{i} \wedge^{\star} A^{j} \tau_{i} \tau_{j}+\frac{i}{2} A^{i} \wedge^{\star} A^{3} \tau_{i}+\frac{i}{2} A^{3} \wedge^{\star} A^{i} \tau_{i}+\frac{i}{2} A^{3} \wedge^{\star} A^{3} \tau_{3} .
$$

Com a decomposição (3.11), as componentes de $F$ se tornam

$$
\begin{aligned}
& F^{a}=l^{-1} d e^{a}+\frac{l^{-1}}{2}\left[\epsilon_{b}^{a} e^{b}, \omega\right]+\frac{i}{2} l^{-1}\left[e^{a}, b\right]_{+}, a, b=0,1, \\
& F^{2}=d \omega-\frac{l^{-2}}{2}\left[e^{0}, e^{1}\right]+\frac{i}{2}[\omega, b]_{+}, \\
& F^{3}=d b-\frac{i l^{-2}}{2} e^{0} \wedge^{\star} e^{0}+\frac{i l^{-2}}{2} e^{1} \wedge^{\star} e^{1}+\frac{i}{2} \omega \wedge^{\star} \omega+\frac{i}{2} b \wedge^{\star} b .
\end{aligned}
$$

Com a decomposição de $\Phi$ e de $F$ em suas componentes da base $\tau_{A}$, a ação $S_{N C J T}$ se torna

$$
\begin{aligned}
S_{N C J T}= & \frac{1}{2} \int \Phi_{A} \star F^{A}=\frac{1}{2} l \int \phi_{0} \star F^{0}+\frac{1}{2} l \int \phi_{1} \star F^{1}+\frac{1}{2} \int \phi \star F^{2}-\frac{1}{2} \int \psi \star F^{3} \\
= & \frac{1}{2} \int \phi_{a} \star\left(d e^{a}+\frac{1}{2}\left[\epsilon_{b}^{a} e^{b}, \omega\right]+\frac{i}{2}\left[e^{a}, b\right]_{+}\right)+\frac{1}{2} \int \phi \star\left(d \omega-\frac{l^{-2}}{2}\left[e^{0}, e^{1}\right]+\frac{i}{2}[\omega, b]_{+}\right) \\
& -\frac{1}{2} \int \psi \star\left(d b-\frac{i l^{-2}}{2} e^{0} \wedge^{\star} e^{0}+\frac{i l^{-2}}{2} e^{1} \wedge^{\star} e^{1}+\frac{i}{2} \omega \wedge^{\star} \omega+\frac{i}{2} b \wedge^{\star} b\right),
\end{aligned}
$$

ou em componentes da base coordenada,

$$
\begin{aligned}
S & =\frac{1}{4} \epsilon^{\mu \nu} \int d^{2} x \phi_{a} \star \frac{1}{2}\left(\partial_{\mu} e_{\nu}^{a}-\partial_{\nu} e_{\mu}^{a}-\frac{1}{2}\left(\left[\omega_{\mu}, \epsilon_{b}^{a} e_{\nu}^{b}\right]_{+}-\left[\omega_{\nu}, \epsilon_{b}^{a} e_{\mu}\right]_{+}\right)+\frac{i}{2}\left(\left[b_{\mu}, e_{\nu}^{a}\right]-\left[b_{\nu}, e_{\mu}^{a}\right]\right)\right) \\
& +\frac{1}{4} \epsilon^{\mu \nu} \int d^{2} x \phi \star \frac{1}{2}\left(\partial_{\mu} \omega_{\nu}-\partial_{\nu} \omega_{\mu}-\frac{l^{-2}}{2}\left(\left[e_{\mu}^{0}, e_{\nu}^{1}\right]_{+}-\left[e_{\nu}^{0}, e_{\mu}^{1}\right]_{+}\right)+\frac{i}{2}\left(\left[\omega_{\mu}, b_{\nu}\right]-\left[\omega_{\nu}, b_{\mu}\right]\right)\right) \\
& -\frac{1}{4} \epsilon^{\mu \nu} \int d^{2} x \psi \star \frac{1}{2}\left(\partial_{\mu} b_{\nu}-\partial_{\nu} b_{\mu}-\frac{i l^{-2}}{2}\left[e_{\mu}^{0}, e_{\nu}^{0}\right]+\frac{i l^{-2}}{2}\left[e_{\mu}^{1}, e_{\nu}^{1}\right]+\frac{i}{2}\left[\omega_{\mu}, \omega_{\nu}\right]+\frac{i}{2}\left[b_{\mu}, b_{\nu}\right]\right) .
\end{aligned}
$$

A expressão acima pode ser reescrita como

$$
S^{(0)}=\frac{1}{4} \int \epsilon^{\mu \nu}\left[\phi_{a b} \star\left(\mathcal{R}^{a b}-\Lambda e^{a} \wedge^{\star} e^{b}\right)-2 \phi_{a} \wedge^{\star} T^{a}\right],
$$

em que

$$
\begin{aligned}
& R_{b}^{a}=d \Omega_{b}^{a}+\frac{1}{2}\left[{\Omega^{a}}_{c}, \Omega_{b}^{c}\right], \\
& T^{a}=d e^{a}+\frac{1}{2}\left[\Omega_{c}^{a}, e^{c}\right]_{+}, \\
& \phi_{a b} \equiv\left(\phi \epsilon_{a b}-i \psi \eta_{a b}\right), \Omega_{b}^{a}=\varepsilon_{b}^{a} \omega+i \delta_{b}^{a} b, \Lambda=-\frac{1}{l^{2}},
\end{aligned}
$$

e interpretamos em (3.13) e (3.14) a curvatura 2-forma e a torsão 2-forma, respectivamente. Note que o segundo termo do lado direito destas expressões difere das expressões análogas na geometria 
diferencial padrão, entretanto, se reduzem a elas no limite comutativo. As equações de movimento para o campo auxiliar $\phi^{a}$ dão $T^{a}=0$, i.e., a condição de torsão nula, e daí que a derivada covariante do referencial móvel $e^{a}$ é nula, $D e^{a}=0$.

A extensão do modelo de Jackiw e Teitelboim de gravitação dilatônica em duas dimensões para um espaço-tempo não comutativo foi feito por meio da extensão da teoria de calibre associada de $S U(1,1)$ para $U(1,1)$. A extensão se deveu ao fato de que o grupo $S U(1,1)$ da teoria comutativa não é preservada pelo produto de Moyal: para $g$ e $h$ na representação fundamental de $S U(1,1)$, i.e., $g$ e $h$ campos matriciais, tem-se que $\operatorname{det} g \star h \neq \operatorname{det} g \star \operatorname{det} h=1$. Como conseqüência, surgem novos campos: um novo campo escalar $\psi$ e um campo de calibre $U(1) b_{\mu}$, e a conexão $\Omega_{b}^{a}$ toma valores em $s o(1,1) \oplus u(1)$, que desacoplam no limite comutativo e estão relacionados à parte de traço de $U(1,1)$.

Em componentes, a ação (3.12) se escreve

$$
S^{(0)}=\frac{1}{4} \int d^{2} x \varepsilon^{\mu \nu}\left[\phi_{a b} \star\left(R_{\mu \nu}^{a b}-2 \Lambda e_{\mu}^{a} \star e_{\nu}^{b}\right)-2 \phi_{a} \star T_{\mu \nu}^{a}\right]
$$

com tensor de curvatura

$$
\begin{aligned}
R_{\mu \nu}^{a b}= & \varepsilon^{a b}\left(\partial_{\mu} \omega_{\nu}-\partial_{\nu} \omega_{\mu}+\frac{i}{2}\left[\omega_{\mu}, b_{\nu}\right]+\frac{i}{2}\left[b_{\mu}, \omega_{\nu}\right]\right) \\
& +\eta^{a b}\left(i \partial_{\mu} b_{\nu}-i \partial_{\nu} b_{\mu}+\frac{1}{2}\left[\omega_{\mu}, \omega_{\nu}\right]-\frac{1}{2}\left[b_{\mu}, b_{\nu}\right]\right)
\end{aligned}
$$

e torsão não-comutativa

$$
\begin{aligned}
T_{\mu \nu}^{a}=\partial_{\mu} e_{\nu}^{a}-\partial_{\nu} e_{\mu}^{a} & +\frac{1}{2} \varepsilon^{a}{ }_{b}\left(\left[\omega_{\mu}, e_{\nu}^{b}\right]_{+}-\left[\omega_{\nu}, e_{\mu}^{b}\right]_{+}\right) \\
& +\frac{i}{2}\left(\left[b_{\mu}, e_{\nu}^{a}\right]-\left[b_{\nu}, e_{\mu}^{a}\right]\right) .
\end{aligned}
$$

Há dois campos dilatônicos, $\phi$ e $\psi$, que estão combinados em

$$
\phi_{a b}:=\phi \varepsilon_{a b}-i \eta_{a b} \psi
$$

Todos os comutadores (denotados por $[\cdot, \cdot])$ e anti-comutadores (denotados por $[\cdot, \cdot]_{+}$) são calculados com o produto estrela de Moyal.

Pode-se escrever (3.15) na forma canônica:

$$
S^{(0)}=\int d^{2} x\left(p^{i} \partial_{0} q_{i}-\lambda^{i} \star G_{i}^{(0)}\right),
$$

em que

$$
\begin{aligned}
& q_{i}=\left(e_{1}^{a}, \omega_{1}, b_{1}\right), \\
& p^{i}=\left(\phi_{a}, \phi,-\psi\right), \\
& \lambda^{i}=\left(e_{0}^{a}, \omega_{0}, b_{0}\right) .
\end{aligned}
$$


Os vínculos são

$$
\begin{aligned}
G_{a}^{(0)}=-\partial_{1} \phi_{a}+ & \frac{1}{2} \varepsilon^{b}{ }_{a}\left[\omega_{1}, \phi_{b}\right]_{+}+\frac{i}{2}\left[\phi_{a}, b_{1}\right] \\
& +\frac{\Lambda}{2}\left(-\varepsilon_{a b}\left[e_{1}^{b}, \phi\right]_{+}+i \eta_{a b}\left[e_{1}^{b}, \psi\right]\right), \\
G_{3}^{(0)}=-\partial_{1} \phi+ & \frac{i}{2}\left[\phi, b_{1}\right]+\frac{i}{2}\left[\psi, \omega_{1}\right]-\frac{1}{2} \varepsilon^{a}{ }_{b}\left[\phi_{a}, e_{1}^{b}\right]_{+}, \\
G_{4}^{(0)}=\partial_{1} \psi- & \frac{i}{2}\left[\psi, b_{1}\right]+\frac{i}{2}\left[\phi, \omega_{1}\right]+\frac{i}{2}\left[\phi_{a}, e_{1}^{a}\right] .
\end{aligned}
$$

É fácil verificar que a álgebra de vínculos fecha, e que os parênteses entre os vínculos são

$$
\begin{aligned}
& \left\{\int \alpha^{a} \star G_{a}^{(0)}, \int \beta^{b} \star G_{b}^{(0)}\right\}= \\
& =-\frac{\Lambda}{2} \int\left(\varepsilon_{a b}\left[\alpha^{a}, \beta^{b}\right]_{+} \star G_{3}^{(0)}+i\left[\alpha_{a}, \beta^{a}\right] \star G_{4}^{(0)}\right) \\
& \left\{\int \alpha \star G_{3}^{(0)}, \int \beta \star G_{3}^{(0)}\right\}=\frac{i}{2} \int[\alpha, \beta] \star G_{4}^{(0)} \\
& \left\{\int \alpha \star G_{4}^{(0)}, \int \beta \star G_{4}^{(0)}\right\}=-\frac{i}{2} \int[\alpha, \beta] \star G_{4}^{(0)} \\
& \left\{\int \alpha \star G_{3}^{(0)}, \int \beta \star G_{4}^{(0)}\right\}=-\frac{i}{2} \int[\alpha, \beta] \star G_{3}^{(0)} \\
& \left\{\int \alpha \star G_{3}^{(0)}, \int \beta^{a} \star G_{a}^{(0)}\right\}=-\frac{1}{2} \int\left[\alpha, \beta^{a}\right]_{+} \varepsilon^{b}{ }_{a} \star G_{b}^{(0)} \\
& \left\{\int \alpha \star G_{4}^{(0)}, \int \beta^{a} \star G_{a}^{(0)}\right\}=-\frac{i}{2} \int\left[\alpha, \beta^{a}\right] \star G_{a}^{(0)}
\end{aligned}
$$

Aqui introduzimos a abreviatura $\int:=\int d^{2} x$. Podemos facilmente encontrar as transformações de calibre gerados por esses vínculos por meio das definições

$$
\begin{aligned}
& \delta p_{a}=\left\{\int d^{D} x \alpha^{i} \star G_{i}, p_{a}\right\} \\
& \delta q^{a}=\left\{\int d^{D} x \alpha^{i} \star G_{i}, q^{a}\right\}
\end{aligned}
$$

em que $\alpha^{i}$ são parâmetros de calibre arbitrários. As transformações geradas por $G_{a}^{(0)}$ são

$$
\begin{aligned}
& \delta e_{\mu}^{a}=-\partial_{\mu} \alpha^{a}-\frac{1}{2} \varepsilon^{a}{ }_{c}\left[\omega_{\mu}, \alpha_{c}\right]_{+}-\frac{i}{2}\left[b_{1}, \alpha^{a}\right], \\
& \delta \omega_{\mu}=\delta b_{\mu}=0, \\
& \delta \phi=\frac{1}{2} \varepsilon^{b}{ }_{a}\left[\alpha^{a}, \phi_{b}\right]_{+}, \quad \delta \psi=-\frac{i}{2}\left[\alpha^{a}, \phi_{a}\right], \\
& \delta \phi_{a}=-\Lambda \alpha^{b} \varepsilon_{b a} .
\end{aligned}
$$


$\mathrm{O}$ vínculo $G_{3}^{(0)}$ gera

$$
\begin{aligned}
& \delta e_{\mu}^{a}=\frac{1}{2} \varepsilon_{b}^{a}\left[e_{\mu}^{b}, \beta\right]_{+}, \quad \delta b_{\mu}=\frac{i}{2}\left[\omega_{\mu}, \beta\right], \\
& \delta \omega_{\mu}=-\partial_{\mu} \beta-\frac{i}{2}\left[b_{\mu}, \beta\right] \\
& \delta \phi_{a}=-\frac{1}{2} \varepsilon^{c}{ }_{a}\left[\beta, \phi_{c}\right]_{+}, \quad \delta \phi=\frac{1}{2}[\beta, \psi], \quad \delta \psi=-\frac{i}{2}[\beta, \phi] .
\end{aligned}
$$

Finalmente, as transformações de calibre geradas por $G_{4}^{(0)}$ são

$$
\begin{aligned}
\delta e_{\mu}^{a} & =-\frac{i}{2}\left[e_{\mu}^{a}, \gamma\right], \quad \delta \omega_{\mu}=-\frac{i}{2}\left[\omega_{\mu}, \gamma\right], \\
\delta b_{\mu} & =-\partial_{\mu} \gamma-\frac{i}{2}\left[b_{\mu}, \gamma\right], \\
\delta \phi_{a} & =\frac{i}{2}\left[\gamma, \phi_{a}\right], \quad \delta \phi=\frac{i}{2}[\gamma, \phi], \quad \delta \psi=\frac{i}{2}[\gamma, \psi] .
\end{aligned}
$$

Em (3.30) - (3.32) as funções $\alpha^{a}, \beta$ e $\gamma$ são parâmetros das transformações de calibre.

As transformações (3.30) e (3.31) são equivalentes às simetrias de difeomorfismos e transformações de Lorentz locais no limite comutativo, a menos de redefinições dos parâmetros das transformações. Já as transformações (3.32) não tem equivalente comutativo, e se desacoplam no limite comutativo. Assim, de acordo com o formalismo canônico apresentado, podemos dizer que as simetrias de calibre da ação (3.19) contem deformações não-comutativas dos grupos de simetria de Lorentz e de difeomorfismos.

\subsection{Deformações}

Consideremos agora deformações do modelo NCJT. Mais termos serão adicionados à ação (3.15) tal que (i) o conteúdo de campos do modelo permanecerá inalterado, e (ii) o número de vínculos primários de primeira classe (e, consequentemente, o número de simetrias de calibre) também permanecerá inalterado. Tendo sido inspirados por modelos de gravitação dilatônica comutativa, apenas consideraremos deformações ao potencial, e somente adicionaremos termos quadráticos nos dois campos dilatônicos $\phi$ e $\psi$.

Além de analogias com o caso comutativo, há também outras razões para não considerarmos deformações dos termos de curvatura e torsão. Por exemplo, substituindo $\phi_{a b}$ em (3.18) por uma função não-linear dos dílatons é equivalente a uma redefinição dos campos dilatônicos. Somar potências mais altas da curvatura em geral adiciona novos graus de liberdade à teoria, e esta seria uma modificação mais drástica àquela que normalmente se entende por deformação. O mesmo se aplica aos termos de torsão.

Restrições adicionais a possíveis deformações são impostas por simetrias globais do modelo, que gostaríamos de preservar. Em primeiro lugar, exige-se simetria com respeito à rotações globais dos índices tangentes e de mundo. Isto implica que todos os índices devem ser contraídos aos pares. Também se exige que os novos termos sejam de paridade par. Como $\phi$ é escalar, e $\psi$ é um pseudoescalar, potências pares (ímpares) de $\psi$ devem ser multiplicadas por potências pares (ímpares) do símbolo de Levi-Civita $\varepsilon$. Como resultado, obtemos a seguinte família de deformações quadráticas do modelo NCJT.

$$
S=S^{(0)}+\tilde{S},
$$


onde

$$
\begin{gathered}
\tilde{S}=\int d^{2} x\left(\varepsilon ^ { \mu \nu } \varepsilon _ { a b } \left(c_{1} e_{\mu}^{a} \star e_{\nu}^{b} \star \phi^{2}+c_{2} e_{\mu}^{a} \star e_{\nu}^{b} \star \psi^{2}\right.\right. \\
\left.+c_{3} e_{\mu}^{a} \star \phi \star e_{\nu}^{b} \star \phi+c_{4} e_{\mu}^{a} \star \psi \star e_{\nu}^{b} \star \psi\right) \\
+\varepsilon^{\mu \nu} \eta_{a b}\left(c_{5} e_{\mu}^{a} \star e_{\nu}^{b} \star[\phi, \psi]+i c_{6} e_{\mu}^{a} \star e_{\nu}^{b} \star[\phi, \psi]_{+}\right. \\
\left.\left.+\frac{i}{2} c_{7}\left(e_{\mu}^{a} \star \phi \star e_{\nu}^{b} \star \psi-e_{\mu}^{a} \star \psi \star e_{\nu}^{b} \star \phi\right)\right)\right) .
\end{gathered}
$$

As constantes arbitrárias $c_{1}, c_{2}, \ldots, c_{7}$ devem ser reais para garantir a realidade da ação total $S$. As potências se referem ao produto estrela, por exemplo $\phi^{2} \equiv \phi \star \phi$.

Os vínculos são

$$
G_{a}=G_{a}^{(0)}+\tilde{G}_{a}, \quad G_{3}=G_{3}^{(0)}, \quad G_{4}=G_{4}^{(0)},
$$

em que

$$
\begin{aligned}
\tilde{G}_{a}= & \varepsilon_{a b}\left(c_{1}\left[e_{1}^{b}, \phi^{2}\right]_{+}+c_{2}\left[e_{1}^{b}, \psi^{2}\right]_{+}+2 c_{3} \phi e_{1}^{b} \phi+2 c_{4} \psi e_{1}^{b} \psi\right) \\
& +\eta_{a b}\left(c_{5}\left[e_{1}^{b},[\phi, \psi]\right]+i c_{6}\left[e_{1}^{b},[\phi, \psi]_{+}\right]+i c_{7}\left(\phi e_{1}^{b} \psi-\psi e_{1}^{b} \phi\right)\right) .
\end{aligned}
$$

Nosso próximo passo é verificar se a álgebra de vínculos ainda fecha na superfície de vínculos ${ }^{6}$. Como os vínculos $G_{3}$ e $G_{4}$ estão inalterados, os colchetes entre eles (3.25) - (3.27) são os mesmos. É um exercício simples verificar que para todos os valores das constantes $c_{m}$

$$
\left\{\int \alpha \star G_{4}, \int \beta^{a} \star \tilde{G}_{a}\right\}=-\frac{i}{2} \int\left[\alpha, \beta^{a}\right] \star \tilde{G}_{a} .
$$

Consequentemente, para quaisquer valores de $c_{m}$ o colchete entre $G_{4}$ e $G_{a}$,

$$
\left\{\int \alpha \star G_{4}, \int \beta^{a} \star G_{a}\right\}=-\frac{i}{2} \int\left[\alpha, \beta^{a}\right] \star G_{a},
$$

é novamente um vínculo no novo conjunto (3.35), de forma que não surgem restrições aos $c_{m}$.

Vamos considerar o colchete entre $G_{3}$ e $G_{a}$,

$$
\begin{aligned}
& \left\{\int \alpha \star G_{3}, \int \beta^{a} \star \tilde{G}_{a}\right\}=\frac{1}{2} \int\left[c _ { 1 } \left(\beta_{a} \star\left[\left[\alpha, e_{1}^{a}\right]_{+}, \phi^{2}\right]_{+}\right.\right. \\
& \left.\quad+i \beta^{a} \star \varepsilon_{a b}\left[e_{1}^{b},[[\alpha, \psi], \phi]_{+}\right]_{+}\right)+c_{2}\left(\beta_{a} \star\left[\left[\alpha, e_{1}^{a}\right]_{+}, \psi^{2}\right]_{+}\right. \\
& \left.\quad-i \beta^{a} \star \varepsilon_{a b}\left[e_{1}^{b},[[\alpha, \phi], \psi]_{+}\right]_{+}\right)+2 c_{3}\left(\beta_{a} \star \phi \star\left[\alpha, e_{1}^{a}\right]_{+} \star \phi\right. \\
& \left.\quad+i \beta^{a} \star \varepsilon_{a b}\left([\alpha, \psi] \star e_{1}^{b} \star \phi+\phi \star e_{1}^{b} \star[\alpha, \psi]\right)\right)+2 c_{4}\left(\beta_{a} \star \psi \star\left[\alpha, e_{1}^{a}\right]_{+} \star \psi\right. \\
& \left.\quad-i \beta^{a} \star \varepsilon_{a b}\left([\alpha, \phi] \star e_{1}^{b} \star \psi+\psi \star e_{1}^{b} \star[\alpha, \phi]\right)\right) \\
& \quad+c_{5}\left(\beta^{a} \star \varepsilon_{a b}\left[\left[\alpha, e_{1}^{b}\right]_{+},[\phi, \psi]\right]+i \beta_{a} \star\left[e_{1}^{a},[[\alpha, \psi], \psi]-[\phi,[\alpha, \phi]]\right]\right) \\
& \quad+i c_{6}\left(\beta^{a} \star \varepsilon_{a b}\left[\left[\alpha, e_{1}^{b}\right]_{+},[\phi, \psi]_{+}\right]+i \beta_{a} \star\left[e_{1}^{a},[[\alpha, \psi], \psi]_{+}-[\phi,[\alpha, \phi]]_{+}\right]\right) \\
& \quad+i c_{7}\left(\beta^{a} \star \varepsilon_{a b}\left(\phi \star\left[\alpha, e_{1}^{b}\right]_{+} \star \psi-\psi \star\left[\alpha, e_{1}^{b}\right]_{+} \star \phi\right)\right. \\
& \left.\left.\quad+i \beta_{a} \star\left([\alpha, \psi] \star e_{1}^{a} \star \psi-\phi \star e_{1}^{a} \star[\alpha, \phi]+[\alpha, \phi] \star e_{1}^{a} \star \phi-\psi \star e_{1}^{a} \star[\alpha, \psi]\right)\right)\right] .
\end{aligned}
$$

\footnotetext{
${ }^{6}$ Em princípio, outras modificações substanciais da álgebra de vínculos podem ocorrer, mas não no caso presente. Nós limitamos o número de simetrias de calibre a quatro, então apenas quatro vínculos de primeira classe são permitidos, porque há apenas quatro pares de variáveis canonicamente conjugadas. Logo, a única possibilidade é que os $G_{i}$ sejam de primeira classe e seus colchetes sejam novamente combinações lineares de $G_{i}$.
} 
Primeiro, observemos que o lado direito de (3.39) não contem termos com derivadas. Isto exclui a possibilidade do colchete (3.39) conter termos proporcionais a (3.21), (3.22), ou (3.23). Logo, esse colchete só pode ser proporcional a (3.36), com coeficientes (constantes de estrutura) como em (3.28), tal que os colchetes entre $G_{3}$ e $G_{a}$ se somam para dar

$$
\left\{\int \alpha \star G_{3}, \int \beta^{a} \star G_{a}\right\}=-\frac{1}{2} \int\left[\alpha, \beta^{a}\right]_{+} \varepsilon^{b}{ }_{a} \star G_{b} .
$$

Temos de comparar as expressões em ambos os lados de (3.40) para obter restrições às constantes $c_{m}$. Não há monômios no lado direito de (3.40) que sejam de segunda ordem em $\phi$ e tenham um fator $i$ explícito. Ao mesmo tempo, há um tal termo proporcional a $c_{5}$ em (3.39). Como todos os $c_{m}$ são reais, conclui-se que

$$
c_{5}=0 \text {. }
$$

Em seguida comparamos os termos em que dois $\phi$ aparecem um ao lado do outro ${ }^{7}$ (combinados em $\left.\phi^{2}\right)$. Esses termos concordam em ambos os lados de (3.40) se e somente se

$$
c_{6}=-c_{1} .
$$

Por comparação dos termos em que dois campos $\phi$ aparecem separados por outros campos, obtém-se a seguinte condição

$$
2 c_{3}=-c_{7} .
$$

Então repetimos o mesmo procedimento com os termos que são quadráticos em $\psi$ para obter

$$
c_{2}=c_{6}, \quad 2 c_{4}=-c_{7} .
$$

A comparação de termos mistos (contendo tanto $\phi$ quanto $\psi$ ) não introduz quaisquer restrições adicionais aos $c_{m}$. Conclui-se que restam apenas duas constantes independentes ( $c_{1}$ e $c_{7}$, digamos), então $\tilde{G}_{a}$ pode ser reescrito como

$$
\begin{aligned}
\tilde{G}_{a}= & c_{1}\left(\varepsilon_{a b}\left[e_{1}^{b}, \phi^{2}-\psi^{2}\right]_{+}-i \eta_{a b}\left[e_{1}^{b},[\phi, \psi]_{+}\right]\right) \\
& +c_{7}\left(-\varepsilon_{a b}\left(\phi \star e_{1}^{b} \star \phi+\psi \star e_{1}^{b} \star \psi\right)+i \eta_{a b}\left(\phi \star e_{1}^{b} \star \psi-\psi \star e_{1}^{b} \star \phi\right)\right) .
\end{aligned}
$$

Ainda resta analisar os colchetes entre $G_{a}$ e $G_{b}$. Obviamente, os colchetes entre $\tilde{G}_{a}$ e $\tilde{G}_{b}$ são nulos, então toda informação relevante está contida nos colchetes entre $G_{a}^{(0)}$ e $\tilde{G}_{b}$. A estratégia é a mesma que antes. Primeiro analisamos os termos contendo derivadas

$$
\begin{aligned}
& \left\{\int \alpha^{a} \star G_{a}^{(0)}, \int \beta^{b} \star \tilde{G}_{b}\right\}+\left\{\int \alpha^{a} \star \tilde{G}_{a}, \int \beta^{b} \star G_{b}^{(0)}\right\}= \\
& =\int\left[c _ { 1 } \left(\partial_{1} \phi \star\left(\left[\phi, \varepsilon_{b c}\left[\beta^{b}, \alpha^{c}\right]_{+}\right]_{+}+i\left[\psi,\left[\alpha_{b}, \beta^{b}\right]\right]_{+}\right)\right.\right. \\
& \left.\quad+\partial_{1} \psi \star\left(-\left[\psi, \varepsilon_{b c}\left[\beta^{b}, \alpha^{c}\right]_{+}\right]_{+}+i\left[\phi,\left[\alpha_{b}, \beta^{b}\right]\right]_{+}\right)\right) \\
& +c_{7}\left(\partial_{1} \phi \star\left(-\varepsilon_{b c}\left(\beta^{b} \star \phi \star \alpha^{c}+\alpha^{c} \star \phi \star \beta^{b}\right)+i\left(\alpha^{b} \star \psi \star \beta_{b}-\beta_{b} \star \psi \star \alpha^{b}\right)\right)\right. \\
& \left.\left.\quad-\partial_{1} \psi \star\left(\varepsilon_{b c}\left(\beta^{b} \star \psi \star \alpha^{c}+\alpha^{c} \star \psi \star \beta^{b}\right)+i\left(\alpha^{b} \star \phi \star \beta_{b}-\beta_{b} \star \phi \star \alpha^{b}\right)\right)\right)\right]
\end{aligned}
$$

+ termos sem derivadas.

\footnotetext{
${ }^{7}$ Isto também inclui os termos que podem ser postos nessa forma usando a propriedade (C.5).
} 
A partir desta equação vemos que o colchete entre $G_{a}$ e $G_{b}$ deve ser uma combinação linear de vínculos (3.35), os vínculos que aparecem no lado direito podem apenas ser $G_{3}$ e $G_{4}$, uma vez que a derivada $\partial_{1} \phi_{a}$ pertencente a $G_{a}$ não está presente. De fato, também é possível obter as funções de estrutura de (3.46), mas sua forma explícita não será necessária. Vamos considerar os termos no colchete que contenham o referencial móvel $e_{1}^{a}$ e o dílaton $\phi$.

$$
\begin{aligned}
& \left\{\int \alpha^{a} \star G_{a}^{(0)}, \int \beta^{b} \star \tilde{G}_{b}\right\}= \\
& =\int\left[\frac{c_{1}}{2}\left(\varepsilon_{b c}\left[\beta^{b}, e_{1}^{c}\right]_{+} \star\left[\phi, \varepsilon_{a}^{d}\left[\alpha^{a}, \phi_{d}\right]_{+}\right]_{+}-\left[\beta_{b}, e_{1}^{b}\right] \star\left[\phi,\left[\alpha^{a}, \phi_{a}\right]\right]_{+}\right)\right. \\
& \quad+\frac{c_{7}}{2}\left(-\varepsilon_{b c} \beta^{b} \star \varepsilon_{a}^{d}\left(\left[\alpha^{a}, \phi_{d}\right]_{+} \star e_{1}^{c} \star \phi+\phi \star e_{1}^{c} \star\left[\alpha^{a}, \phi_{d}\right]_{+}\right)\right. \\
& \left.\left.\quad+\beta_{b}\left(\phi \star e_{1}^{b} \star\left[\alpha^{a}, \phi_{a}\right]-\left[\alpha^{a}, \phi_{a}\right] \star e_{1}^{b} \star \phi\right)\right)\right] \\
& \quad+\text { termos sem } e_{1}^{b} \text { ou } \phi .
\end{aligned}
$$

Os argumentos apresentados acima mostram que se o colchete (3.46) fecha para os vínculos existentes, esses vínculos são $G_{3}$ e $G_{4}$, e as funções de estrutura dependem de $\phi$ e $\psi$. Para ambos $G_{3}$ e $G_{4}$ os campos $e_{1}^{a}$ e $\phi_{b}$ surgem nas combinações $\left[\phi_{a}, e_{1}^{b}\right]$ ou $\left[\phi_{a}, e_{1}^{b}\right]_{+}$, i.e. eles permanecem juntos. Logo, todos os termos em que $\phi_{b}$ e $e_{1}^{a}$ surgem separados por outros campos devem ser nulos. Vamos verificar se isto pode ser obtido pelo ajuste dos parâmetros $c_{1}$ e $c_{7}$ restantes. Consideremos os termos com $\phi, \phi_{0}, \alpha^{0}, \beta^{0}, e_{1}^{0}$ em que $\alpha^{0}$ e $\beta^{0}$ ficam juntos, mas $\phi_{0}$ e $e_{1}^{0}$ estão separados. Todos os termos dessa forma em (3.46) podem facilmente ser reunidos com a ajuda de (3.47). Eles são

$$
\int \frac{c_{1}}{2}\left[\alpha^{0}, \beta^{0}\right] \star\left(\phi_{0} \star \phi \star e_{1}^{0}-e_{1}^{0} \star \phi \star \phi_{0}\right) .
$$

Como eles não são permitidos, conclui-se que

$$
c_{1}=0
$$

Vamos agora reunir todos os termos com os mesmos componentes de campos onde novamente $\phi_{0} \mathrm{e}$ $e_{1}^{0}$ estão separados, mas sem quaisquer restrições sobre $\alpha^{0}$ e $\beta^{0}$.

$$
\int \frac{c_{7}}{2}\left[e_{1}^{0}, \phi\right] \star\left(\beta^{0} \star \phi_{0} \star \alpha^{0}-\alpha^{0} \star \phi_{0} \star \beta^{0}\right) .
$$

Tais termos também não são permitidos. Logo,

$$
c_{7}=0 .
$$

Acabamos de demonstrar que não há deformação quadrática consistente do modelo NCJT. Isto significa que o modelo NCJT é estável ante tais deformações.

\subsection{Conclusões}

Neste capítulo estudamos se é possível deformar a ação do modelo NCJT pela adição de termos quadráticos ao potencial dilatônico enquanto se preserva o número de vínculos de primeira classe. A resposta que obtivemos é negativa. Isto, é claro, não exclui a existência de modelos de gravitação 
NC interessantes. Ainda há a possibilidade da existência de outras teorias de gravitação dilatônica $\mathrm{NC}$ interagentes com simetrias de calibre usuais (não-torcidas). Entretanto, é claro que a maioria dos modelos de gravitação dilatônica (que admitem potenciais dilatônicos arbitrários) não podem ser estendidos a espaços-tempo não-comutativos usando esta abordagem. Logo, nossos resultados podem ser considerados um forte argumento a favor da abordagem torcida [93], que permite praticamente auto-interações arbitrárias entre os campos escalares. Também notamos que alguns resultados anteriores [120] que mostram que deformações de teorias de gravitação 2D são triviais se não se introduz certa quantidade de estrutura de grupos quânticos. Um outro resultado importante é a construção de simetrias conformes torcidas em duas dimensões [121]. Para incorporar simetrias torcidas no formalismo canônico, deve-se provavelmente incluir torções no próprio formalismo canônico.

Finalmente, como a redução esférica de teorias de gravitação de Einstein em dimensões elevadas produzem algumas teorias de gravitação dilatônica em duas dimensões, pode-se esperar que nosso resultado no-go possa ser estendido de alguma forma a dimensões mais altas. 


\section{Capítulo 4}

\section{Não-comutatividade nilpotente e renormalização}

\subsection{Introdução}

É bem sabido [122] que teorias de campo não-comutativas (NC) têm problemas de renormalizibilidade devido à chamada mistura UV/IR [14, 15, 16]. Para superar essa dificuldade, modifica-se o propagador pela adição de um termo oscilante [17, 18, 19] com o fim de respeitar a dualidade de Langmann-Szabo [20], ou pela adição de um termo com uma potência negativa do momento [123]. Supersimetria também melhora as propriedades de renormalização de teorias NC (veja, e.g., [124]). Algumas versões de supersimetria NC (aquelas baseadas no superespaço não-anti-comutativo $[125,126]$, veja também $[127,128]$ ) têm um parâmetro NC, tal que o produto estrela termina em uma ordem finita de sua expansão. Foi demonstrado [129] que a presença de um parâmetro nilpotente NC não necessariamente implica supersimetria. Em [129] um parâmetro nilpotente (bifermiônico) $\mathrm{NC}$ foi introduzido numa teoria bosônica, dando lugar a muitas das propriedades atraentes daquele modelo. O objetivo desse trabalho é estudar até que ponto a presença de um parâmetro NC nilpotente (ou bifermiônico) influencia a renormalização. Nós não iremos considerar teorias supersimétricas com o intuito de separar efeitos advindos da nilpotência do parâmetro NC daqueles oriundos de supersimetria.

Um esquema conveniente para tal análise foi sugerido em [129], onde foi proposto considerar um parâmetro NC bifermiônico

$$
\Theta^{\mu \nu}=i \theta^{\mu} \theta^{\nu}
$$

em que $\theta^{\mu}$ é real, constante e fermiônico (uma constante de Grassmann ímpar), $\theta^{\mu} \theta^{\nu}=-\theta^{\nu} \theta^{\mu}$. Os (anti)comutadores da álgebra $\mathrm{NC}$

$$
\left[x^{\mu}, x^{\nu}\right]=i \theta^{\mu} \theta^{\nu},\left\{\theta^{\mu}, \theta^{\nu}\right\}=0,\left[x^{\mu}, \theta^{\nu}\right]=0
$$

satisfazem as identidades de Jacobi graduadas. Note que constantes bifermiônicas surgem naturalmente em modelos pseudoclássicos de partículas relativísticas [130, 42]. Devido à anti-comutatividade de $\theta^{\mu}$, a expansão do produto de Moyal termina no segundo termo,

$$
f_{1} \star f_{2}=\left.\exp \left(\frac{i}{2} \Theta^{\mu \nu} \partial_{\mu}^{x} \partial_{\nu}^{y}\right) f_{1}(x) f_{2}(y)\right|_{y=x}=f_{1} f_{2}-\frac{1}{2} \theta^{\mu} \theta^{\nu} \partial_{\mu} f_{1} \partial_{\nu} f_{2} .
$$


O produto estrela, portanto, se torna local.

Em [129] um parâmetro NC bifermiônico foi usado para construir um modelo de teoria de campos em duas dimensões que, ao contrário aos modelos NC tipo tempo-espaço usuais, tem um tensor de energia e momento conservado localmente, um hamiltoniano conservado bem-definido, e pode ser quantizado canonicamente sem dificuldades. Ademais, o modelo parece ser renormalizável. No presente capítulo, nós investigamos se não-comutatividade bifermiônica ajuda a renormalizar teorias em quatro dimensões.

Primeiro exploramos um modelo que é a versão quadridimensional do modelo sugerido em [129] (isto não é outra coisa senão $\varphi^{4} \mathrm{NC}$ com um termo de interação adicional para tornar o modelo menos trivial). Resulta que, com um parâmetro NC bifermiônico, esse modelo se torna renormalizável em todas as ordens da expansão em loops. Também estudamos as equações de grupo de renormalização a um loop e encontramos um ponto fixo estável infra-vermelho onde todos as constantes de acoplamento se anulam.

De um ponto de vista técnico, ter um parâmetro NC bifermiônico parecer similar a expandir a teoria em $\Theta$ e manter apenas alguns termos de ordem mais baixa. As propriedades ultravioletas da teoria expandida e completa são bastante diferentes, e, algumas vezes, teorias expandidas tem um comportamento pior (veja, e.g., [131]). A razão é que, de um lado, o propagador em teorias expandidas não tem um comportamento oscilatório, e de outro lado, surgem perigosos vértices dependentes do momento. Todos esses problemas também surgem em teorias com não-comutatividade bifermiônica, mas há um efeito que melhora o comportamento ultravioleta. De fato, alguns termos se anulam, pois $\theta^{2}=0$. Aqui tomamos eletrodinâmica $\mathrm{NC}$ (que é renormalizável a um loop se o parâmetro NC padrão é usado) e demonstramos que com um parâmetro NC bifermiônico esse modelo permanece renormalizável, pelo menos para diagramas a um loop com fótons externos.

\subsection{Um modelo de campo escalar}

A ação do modelo de que tratamos nessa seção se lê

$$
\begin{array}{rl}
S=\int d^{4} & x\left(\frac{1}{2}\left(\partial_{\mu} \varphi_{1}\right)^{2}+\frac{1}{2}\left(\partial_{\mu} \varphi_{2}\right)^{2}+\frac{1}{2}\left(\partial_{\mu} \varphi\right)^{2}-\frac{1}{2} m_{1}^{2} \varphi_{1}^{2}-\frac{1}{2} m_{2}^{2} \varphi_{2}^{2}-\frac{1}{2} m^{2} \varphi^{2}\right. \\
& \left.-\frac{e i}{2}\left[\varphi_{1}, \varphi_{2}\right]_{\star} \star \varphi \star \varphi-\frac{\lambda}{24} \varphi_{\star}^{4}\right),
\end{array}
$$

que é uma versão quadridimensional do modelo sugerido em [129]. As motivações por trás dessa forma particular do modelo são as seguintes. Como qualquer produto estrela simetrizado com parâmetro bifermiônico é equivalente ao produto comutativo usual de funções, precisamos de pelo menos dois campos, $\varphi_{1}$ e $\varphi_{2}$, para construir um termo de interação polinomial não-trivial ${ }^{1}$. Como foi explicado em [129], mesmo dois campos não são o bastante, então nós incluímos outro campo escalar $\varphi$ para construir o termo de interação com constante de acoplamento $e$. Adicionou-se um termo de auto-interação $\varphi_{\star}^{4}=\varphi \star \varphi \star \varphi \star \varphi$ para tornar a dinâmica mais interessante. $e$ e $\lambda$ são constantes de acoplamento reais.

Em [129] foi demonstrado que um modelo em duas dimensões com a mesma densidade lagrangiana que em (4.3) é renormalizável. É relativamente fácil conseguir renormalizibilidade em duas dimensões. Por exemplo, há um modelo de gravitação $\mathrm{NC}$ em duas dimensões para o qual

\footnotetext{
${ }^{1}$ Por exemplo, usando o produto de estrela bifermiônico e integrando por partes, é fácil ver que $\int d^{4} x \phi \star \bar{\phi} \star \phi \star \phi=$ $\int d^{4} x \phi \phi \bar{\phi} \bar{\phi}$.
} 
todo o funcional gerador quântico de funções de Green pode ser calculado não-perturbativamente em todas as ordens da expansão em loops [98] por meio de métodos desenvolvidos anteriormente para o caso comutativo [117]. Aqui, para se estar mais próximo da física, nós consideramos um modelo quadridimensional (4.3).

Devido à escolha (4.1) do parâmetro NC, a parte de interação da ação (4.3) tem uma forma bastante simples,

$$
S_{\mathrm{int}}=\int d^{4} x\left(\frac{e i}{2}\left(\theta^{\mu} \partial_{\mu} \varphi_{1}\right)\left(\theta^{\nu} \partial_{\nu} \varphi_{2}\right) \varphi^{2}-\frac{\lambda}{24} \varphi^{4}\right) .
$$

Agora estamos prontos para deduzir as regras de Feynman para nosso modelo. Os propagadores são os propagadores padrões de campos escalares massivos. Há dois vértices, o vértice padrão $\varphi^{4} \mathrm{e}$ um novo vértice, que depende do parâmetro NC (veja Fig.1).
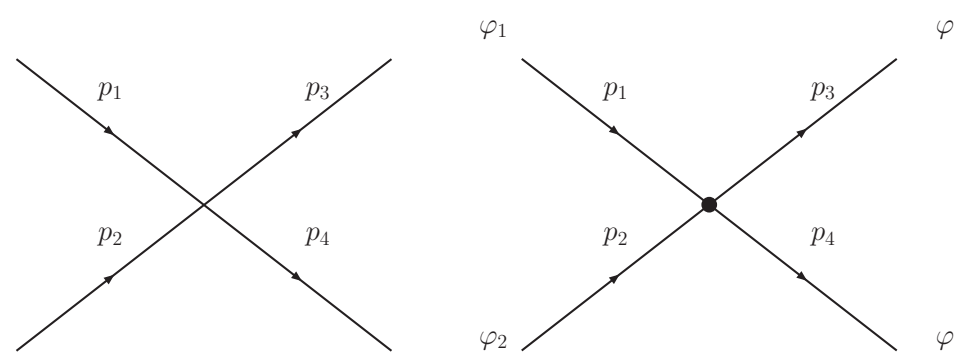

Figura 4.1: $\mathrm{O}$ vértice padrão $\varphi^{4}$ e o novo vértice $-\frac{i e}{2} \theta p_{1} \theta p_{2}$.

A observação principal que prova a renormalizibilidade de (4.3) é que qualquer diagrama com uma linha interna do campo $\varphi_{1}$ ou $\varphi_{2}$ se anula. De fato, qualquer linha interna desses campos inevitavelmente conecta dois 'novos' vértices e, portanto, recebe um fator $(\theta \cdot k)^{2}=0$, onde $k$ é o momento correspondente. Renormalizibilidade por contagem de potências do nosso modelo segue então por razões usuais, exatamente como no caso comutativo. Considere um diagrama com $N$ vértices e $2 K$ pernas externas. Esse diagrama tem $\frac{1}{2}(4 N-2 K)=2 N-K$ linhas internas, dando uma potência do momento total no integrando de $-2(2 N-K)$. Os momentos das linhas internas estão restritos por $N-1$ funções-delta, em que -1 corresponde à conservação do momento total de todas as pernas externas. Juntando tudo, obtém-se um grau de divergência de $4-2 K$, como no caso da teoria $\varphi^{4}$ comutativa. Os diagramas divergentes por contagem de potências são aqueles com duas ou quatro pernas externas. Os diagramas contendo apenas pernas $\varphi$ são precisamente os mesmos que aqueles no caso comutativo, e eles são renormalizados precisamente do mesmo modo. Vamos considerar os diagramas com pernas $\varphi_{1}$ e $\varphi_{2}$. Há três tipos de tais diagramas (veja Fig. 4.2) 

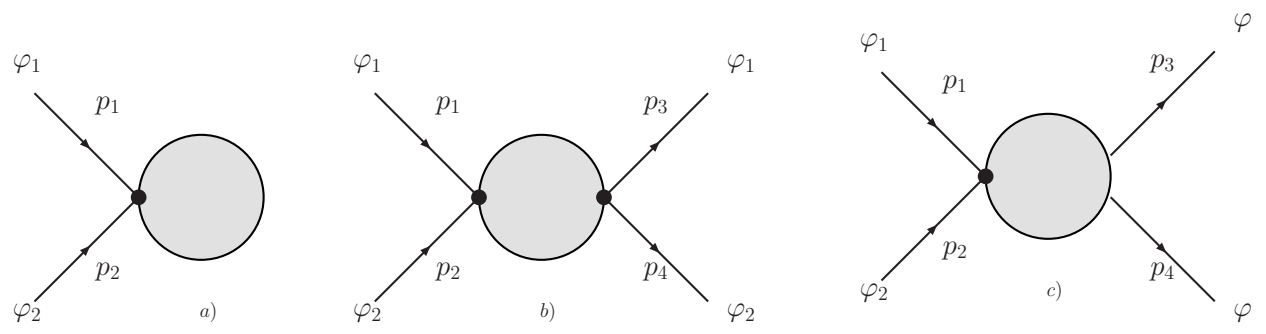

Figura 4.2: Os três diagramas divergentes.

O diagrama na Fig. 4.2a é proporcional a $(p \theta)^{2}$, e, portanto, se anula. $\mathrm{O}$ diagrama na Fig. $4.2 \mathrm{~b}$ contem $\left(p_{1} \theta\right)\left(p_{2} \theta\right)\left(p_{3} \theta\right)\left(p_{4} \theta\right)=0$, devido à conservação de momento, $p_{1}+p_{2}=p_{3}+p_{4}$. O diagrama da Fig. 4.2c tem divergência no máximo logarítmica. Logo, suas partes divergentes são proporcionais à potência mais baixa dos momentos externos, i.e., a $\left(p_{1} \theta\right)\left(p_{2} \theta\right)$. É fácil ver que tais divergências podem ser removidas por uma renormalização do acoplamento e na ação (4.3). Nós concluímos que o modelo (4.3) com parâmetro NC bifermiônico é renormalizável em todas as ordens da expansão em loops.

A renormalização de todos parâmetros relacionados ao campo $\varphi$ (a renormalização de $m, \lambda$ e da função de onda $\varphi$ ) não é sensível à presença dos outros campos $\varphi_{1}$ e $\varphi_{2}$. Não há renormalização da massa ou da função de onda para $\varphi_{1}$ ou $\varphi_{2}$. Pela comparação de fatores combinatórios que surgem em frente aos diagramas de Feynman relevantes, e usando o resultado padrão [132] para teoria $\varphi^{4}$ comutativa no esquema de regularização dimensional, pode-se deduzir a relação

$$
3 \frac{\delta e}{e}=\frac{\delta \lambda}{\lambda}=\frac{\lambda}{16 \pi^{2}} \frac{3}{\epsilon}
$$

entre renormalizações infinitas a um loop das cargas e e $\lambda$. A função $\beta$ para $\lambda$ é bem conhecida $[132]$

$$
\beta_{\lambda}=-\epsilon \lambda+\frac{3 \lambda^{2}}{16 \pi^{2}}+O\left(\lambda^{3}\right)
$$

Da relação (4.5), pode-se obter a dimensão anômala do acoplamento $e, \beta_{e}$, usando o fato de que o acoplamento nu (bare) é invariante por transformações do grupo de renormalização,

$$
\mu \frac{d e_{0}}{d \mu}=0, e_{0}=\mu^{\epsilon} e\left(1+\frac{\lambda}{16 \pi^{2}} \frac{1}{\epsilon}\right) \text {. }
$$

Explicitamente,

$$
\mu \frac{d}{d \mu} e_{0}=\mu^{\epsilon}\left(\epsilon e+\frac{e \lambda}{16 \pi^{2}}\right)+\mu^{\epsilon}\left[\beta_{e}\left(1+\frac{\lambda}{16 \pi^{2}} \frac{1}{\epsilon}\right)+\frac{e}{16 \pi^{2}} \frac{1}{\epsilon} \beta_{\lambda}\right]=0,
$$

que implica

$$
\begin{aligned}
\beta_{e} & =-\left[\epsilon e+\frac{e \lambda}{16 \pi^{2}}+\frac{e}{16 \pi^{2}} \frac{1}{\epsilon} \beta_{\lambda}\right]\left(1-\frac{\lambda}{16 \pi^{2}} \frac{1}{\epsilon}\right) \\
& =-\epsilon e+\frac{\lambda e}{16 \pi^{2}}+O\left(e \lambda^{2}\right) .
\end{aligned}
$$


Agora podemos remover a regularização impondo $\epsilon=0$ e resolver as equações de grupo de renormalização

$$
\mu \frac{d}{d \mu} \lambda(\mu)=\beta_{\lambda}(\lambda(\mu)), \quad \mu \frac{d}{d \mu} e(\mu)=\beta_{e}(e(\mu))
$$

para os acoplamentos (running) $\lambda(\mu)$ e $e(\mu)$. As condições iniciais são $\lambda\left(\mu_{0}\right)=\lambda, e\left(\mu_{0}\right)=e$ com $\mu_{0}$ sendo a escala de normalização. Como $\beta_{\lambda}$ não depende de $e$, a equação para $\lambda(\mu)$ pode ser resolvida primeiro, dando o resultado bem conhecido

$$
\lambda(\mu)=\lambda\left(1-\frac{3}{16 \pi^{2}} \lambda \ln \frac{\mu}{\mu_{0}}\right)^{-1} .
$$

Resolvendo então a equação para $e(\mu)$ obtemos

$$
e(\mu)=e\left(1-\frac{3 \lambda}{16 \pi^{2}} \ln \frac{\mu}{\mu_{0}}\right)^{-\frac{1}{3}} .
$$

No limite $\mu \rightarrow 0$ os dois acoplamentos se anulam, e há assim um ponto fixo estável infravermelho. Note que $e(\mu)$ se anula mais lentamente que $\lambda(\mu)$ na vizinhança do ponto fixo.

\subsection{Eletrodinâmica não-comutativa com parâmetro bifermiônico}

Vamos considerar a eletrodinâmica NC no espaço euclidiano com a ação clássica

$$
S_{c l}=\int d^{4} x\left[\frac{1}{4 g^{2}} \hat{F}_{\mu \nu}^{2}+\bar{\psi}\left(i \gamma_{\mu} D_{\mu}\right) \psi\right]
$$

em que $D_{\mu} \psi=\partial_{\mu} \psi-i A_{\mu} \star \psi \mathrm{e}$

$$
\hat{F}_{\mu \nu}=F_{\mu \nu}-i\left(A_{\mu} \star A_{\nu}-A_{\nu} \star A_{\mu}\right), \quad F_{\mu \nu}=\partial_{\mu} A_{\nu}-\partial_{\nu} A_{\mu} .
$$

As matrizes $\gamma$ satisfazem $\left\{\gamma_{\mu}, \gamma_{\nu}\right\}=2 \delta_{\mu \nu}$ e são hermitianas, $\delta_{\mu \nu}=\operatorname{diag}(1,1,1,1)$. Para um parâmetro NC usual, essa teoria é sabidamente renormalizável a um loop [133, 134]. Mas sua expansão em $\Theta$ pode violar sua renormalizibilidade já a um loop, como foi demonstrado por [131] no contexto do mapa de Seiberg-Witten.

Aqui verificaremos se a eletrodinâmica NC permanece renormalizável a um loop se o parâmetro NC é bifermiônico (4.1). Para simplificar a análise se considerará o caso em que apenas $\psi$ é quantizado, ao passo que $A_{\mu}$ permanece um campo clássico de fundo. Pode-se verificar que isso corresponde à retenção de todos os diagramas com fótons externos no calibre de Lorentz. Renormalizibilidade em tal modelo simplificado significa que a divergência a um loop é proporcional ao termo correspondente na ação clássica (4.10), isto é, a $\hat{F}_{\mu \nu}^{2}$. A ação efetiva pode ser formalmente escrita como

$$
W=-\ln \operatorname{det} \not D=-\frac{1}{2} \ln \operatorname{det} \not D^{2}
$$

onde $\not D$ é o operador de Dirac no $\mathbb{R}^{4}$ não-comutativo na presença do campo eletromagnético externo.

$$
\not D=i \gamma_{\mu}\left(\partial_{\mu}-i A_{\mu} \star\right)=i \gamma_{\mu}\left(\partial_{\mu}-i A_{\mu}+\frac{i}{2} \theta \partial A_{\mu} \theta \partial\right), \theta \partial \equiv \theta_{\mu} \partial_{\mu} .
$$


Para evitar o uso excessivo de parênteses, adota-se a convenção de que a derivada age apenas na função adjacente (ignorando, é claro, qualquer número de $\theta$ 's ou outras derivadas que possam surgir no meio). Por exemplo, $\theta \partial A_{\mu} \theta \partial=\left(\theta \partial A_{\mu}\right) \theta \partial$ é um operador diferencial de primeira ordem.

É conveniente utilizar regularização por função zeta de determinantes funcionais [135, 136], de tal forma que a ação efetiva regularizada (4.11) se leia $W^{\text {reg }}=\frac{1}{2} \zeta\left(\not D^{2}, s\right) \Gamma(s)$ onde $\zeta\left(\not D^{2}, s\right)=$ $\operatorname{Tr}_{L^{2}}\left(\left(\not D^{2}\right)^{-s}\right)$. No limite físico, $s \rightarrow 0$, a ação efetiva regularizada diverge, e a parte divergente se lê

$$
W^{\operatorname{div}}=\frac{1}{2 s} \zeta\left(\not D^{2}, 0\right)
$$

Normalmente, $\not D^{2}$ é um operador tipo Laplace, de forma que o traço de calor

$$
K\left(\not D^{2} ; t\right)=\operatorname{Tr}_{L^{2}}\left(e^{-t \not D^{2}}\right)
$$

existe e admite uma expansão assintótica

$$
K\left(\not D^{2} ; t\right) \simeq \sum_{k \geq 0} t^{(k-n) / 2} a_{k}\left(\not D^{2}\right)
$$

à medida que $t \rightarrow+0$. Aqui $n$ é a dimensão da variedade subjacente. Uma revisão da expansão do kernel de calor pode ser encontrada em [137] para variedades comutativas, e em [138] para o caso NC. Vamos assumir que a expansão (4.15) é válida para o operador (4.12). (Isto será demonstrado em um momento). Assim, usando a transformada de Mellin, pode-se mostrar que

$$
\zeta\left(\not D^{2}, 0\right)=a_{4}\left(\not D^{2}\right)
$$

em $n=4$ dimensões. Não há uma boa teoria espectral para operadores diferenciais com símbolos dependentes de parâmetros fermiônicos. Por segurança, vamos calcular (4.16) por dois métodos distintos.

Primeiro, usando resultados existentes para expansão de kernel de calor em variedades NC. O operador

$$
\not D^{2}=-\left(\left(\partial_{\mu}-i A_{\mu} \star\right)^{2}-\frac{i}{4}\left[\gamma^{\mu}, \gamma^{\nu}\right] \hat{F}_{\mu \nu} \star\right),
$$

(em que derivadas parciais agem à direita até o fim), tem apenas produtos estrelas à esquerda (o que significa que na equação de autovalor $\not D^{2} \psi=\lambda \psi$ todos os campos de fundo multiplicam $\psi$ pela esquerda), e portanto, se encaixa na categoria considerada em [139, 140]. Os cálculos feitos em $[139]^{2}$ são regulares em $\Theta=0$ e sobrevivem a uma expansão até uma ordem finita em $\Theta$ (veja eqs.(15) - (26) lá). Note que tal asserção não é válida para operadores que contêm produtos estrela tanto à direita quanto à esquerda [141, 142]. De todo modo, estamos autorizados a utilizar os resultados de $[139,140]$ para o operador $(4.17)$. Primeiro, traz-se $\not D^{2}$ à forma padrão

$$
\not D^{2}=-\left(\hat{\nabla}_{\mu} \hat{\nabla}_{\mu}+\hat{E}_{\star}\right), \quad \hat{\nabla}_{\mu} \equiv \partial_{\mu}+\hat{\omega}_{\mu} \star,
$$

onde

$$
\hat{\omega}_{\mu}=-i A_{\mu}, \quad \hat{E}=-\frac{i}{4}\left[\gamma^{\mu}, \gamma^{\nu}\right] \hat{F}_{\mu \nu} .
$$

\footnotetext{
${ }^{2} \mathrm{O}$ artigo [139] tratou o caso de um toro $\mathrm{NC}$, e o caso do plano $\mathrm{NC}$ foi feito em [140]. No presente contexto, distinções entre o toro e o plano não são essenciais.
} 
Então, de acordo com [139, 140], a expansão assintótica (4.15) existe e o coeficiente $a_{4}$ se lê

$$
a_{4}=\frac{1}{(4 \pi)^{2}} \frac{1}{12} \int d^{4} x \operatorname{tr}\left(6 \hat{E} \star \hat{E}+\hat{\Omega}_{\mu \nu} \star \hat{\Omega}_{\mu \nu}\right)
$$

com $\hat{\Omega}_{\mu \nu}=\left[\hat{\nabla}_{\mu}, \hat{\nabla}_{\nu}\right]$. Substituindo (4.19) em (4.20) e tomando o traço, obtém-se

$$
a_{4}\left(\not D^{2}\right)=\frac{1}{(4 \pi)^{2}} \frac{2}{3} \int d^{4} x \hat{F}_{\mu \nu} \star \hat{F}_{\mu \nu} .
$$

O outro método usado não depende da estrutura do produto estrela, mas sim da forma expandida do operador

$$
\begin{aligned}
\not D^{2} & =-\left(\partial^{2}-2 i A_{\mu} \partial_{\mu}-i\left(\partial_{\mu} A_{\mu}\right)-A^{2}\right)-i(\theta \partial) A^{\mu}(\theta \partial) \partial_{\mu} \\
& -\frac{i}{8}\left[\gamma^{\mu}, \gamma^{\nu}\right](\theta \partial) F_{\mu \nu}(\theta \partial)-\frac{i}{2}(\theta \partial) \partial^{\mu} A_{\mu}(\theta \partial)-A^{\mu}(\theta \partial) A_{\mu}(\theta \partial) \\
& -\frac{1}{4}\left[\gamma^{\mu}, \gamma^{\nu}\right](\theta \partial) A_{\mu}(\theta \partial) A_{\nu}+\frac{i}{4}\left[\gamma^{\mu}, \gamma^{\nu}\right] F_{\mu \nu} .
\end{aligned}
$$

O coeficiente $a_{4}$ pode ser obtido do trabalho seminal de Gilkey [143] pela identificação dos invariantes correspondentes. Para um operador tipo Laplace da forma

$$
P=-\left(g^{\mu \nu} \partial_{\mu} \partial_{\nu}+a^{\sigma} \partial_{\sigma}+b\right)
$$

identifica-se $g^{\mu \nu}$ com a métrica riemanniana (para que se possa fazer tal identificação é necessário que o símbolo inicial seja uma matriz unitária nos índices espinoriais - uma propriedade que é felizmente satisfeita pelo operador (4.22)). Há uma única conexão $\omega$ tal que $P$ possa ser apresentado como

$$
P=-\left(g^{\mu \nu} \nabla_{\mu} \nabla_{\nu}+E\right)
$$

em que a derivada covariante $\nabla=\nabla^{[R]}+\omega$ contem uma conexão riemanniana e uma parte de calibre. A parte de ordem zero se escreve $E=b-g^{\mu \nu}\left(\partial_{\mu} \omega_{\nu}+\omega_{\nu} \omega_{\mu}-\omega_{\sigma} \Gamma_{\nu \mu}^{\sigma}\right)$, onde $\Gamma_{\nu \mu}^{\sigma}$ são os símbolos de Christoffel para a métrica $g^{\mu \nu}$. Pode-se também introduzir o tensor de curvatura $\Omega_{\mu \nu}=\partial_{\mu} \omega_{\nu}-\partial_{\nu} \omega_{\mu}+\left[\omega_{\mu}, \omega_{\nu}\right]$.

Em $n=4$ os coeficientes de kernel de calor relevantes são

$$
a_{4}(P)=\frac{1}{(4 \pi)^{2}} \frac{1}{12} \int d^{4} x \sqrt{g(x)} \operatorname{tr}\left(6 E^{2}+\Omega_{\mu \nu} \Omega_{\rho \sigma} g^{\mu \rho} g^{\nu \sigma}+\left[R^{2}-\text { terms }\right]\right) .
$$

Os termos quadráticos na curvatura de Riemann não foram escritos explicitamente. O modelo foi inicialmente formulado no espaço euclidiano plano, então não há distinções entre índices superiores e inferiores. Sempre que for necessário contrair um par de índices com a métrica efetiva $g^{\mu \nu}$, a métrica estará explícita.

Vamos nos restringir aos termos de ordem zero e de ordem dois em $\theta$. De eq.(4.22) se pode ler a métrica $g^{\mu \nu}$

$$
g^{\mu \nu}=\delta^{\mu \nu}+\frac{i}{2} \theta \partial\left(A^{\mu} \theta^{\nu}+A^{\nu} \theta^{\mu}\right), g_{\mu \nu}=\delta_{\mu \nu}-\frac{i}{2} \theta \partial\left(A_{\mu} \theta_{\nu}+A_{\nu} \theta_{\mu}\right),
$$

o símbolo de Christoffel

$$
\Gamma_{\nu \sigma}^{\mu}=\frac{i}{4} \delta^{\mu \kappa} \theta \partial\left[\theta_{\sigma} F_{\kappa \nu}+\theta_{\nu} F_{\kappa \sigma}-\theta_{\kappa}\left(\partial_{\sigma} A_{\nu}+\partial_{\nu} A_{\sigma}\right)\right]
$$


e $a^{\mu}$ e $b$,

$$
\begin{aligned}
& a^{\mu}=\frac{i}{8}\left[\gamma_{\kappa}, \gamma_{\nu}\right] \theta \partial F_{\kappa \nu} \theta^{\mu}+\frac{i}{2} \theta \partial \partial_{\nu} A_{\nu} \theta^{\mu}+A_{\nu} \theta \partial A_{\nu} \theta^{\mu}-2 i A^{\mu} \\
& b=\frac{1}{4}\left[\gamma_{\mu}, \gamma_{\nu}\right] \theta \partial A_{\mu} \theta \partial A_{\nu}-\frac{i}{4}\left[\gamma_{\mu}, \gamma_{\nu}\right] F_{\mu \nu}-i \partial A-A^{2} .
\end{aligned}
$$

A partir dessas expressões podemos calcular a conexão de calibre

$$
\begin{aligned}
\omega_{\mu} & =\frac{1}{2} g_{\mu \nu}\left(a^{\nu}+g^{\kappa \sigma} \Gamma_{\kappa \sigma}^{\nu}\right) \\
& =-i A_{\mu}-\frac{1}{2}(\theta \partial) A_{\mu}(\theta A)+\frac{i}{4}(\theta \partial) \theta^{\kappa} F_{\mu \kappa}+\frac{i}{16}\left[\gamma^{\kappa}, \gamma^{\sigma}\right] \theta \partial F_{\kappa \sigma} \theta_{\mu},
\end{aligned}
$$

e o traço de $E^{2}$ e $\Omega^{2}$ seguem

$$
\begin{aligned}
& \operatorname{tr} E^{2}=2 \hat{F}_{\mu \nu} \hat{F}_{\mu \nu}+2 i F_{\mu \nu}(\theta A)(\theta \partial) F_{\mu \nu}, \hat{F}_{\mu \nu}=F_{\mu \nu}+i(\theta \partial) A_{\mu}(\theta \partial) A_{\nu}, \\
& \operatorname{tr} g^{\mu \kappa} g^{\nu \sigma} \Omega_{\mu \nu} \Omega_{\kappa \sigma}=-4 \hat{F}_{\mu \nu} \hat{F}_{\mu \nu}+4 i F_{\mu \nu}(\theta \partial) F_{\mu \nu}(\theta A) .
\end{aligned}
$$

O tensor de Riemann para a métrica (4.26) é pelo menos de segunda ordem em $\theta$. Logo, os termos quadráticos na curvatura são pelo menos de quarta ordem em $\theta$ e devem ser desprezados.

Finalmente, somos capazes de calcular $a_{4}$,

$$
a_{4}\left(\not D^{2}\right)=\frac{1}{(4 \pi)^{2}} \frac{2}{3} \int d^{4} x \hat{F}_{\mu \nu} \hat{F}_{\mu \nu},
$$

que está em acordo com (4.21).

Os dois métodos usados acima para calcular o coeficiente do kernel de calor $a_{4}$ diferem quanto ao modo como foram tratadas as derivadas contidas no produto estrela. No segundo método essas derivadas modificam os termos de primeira e segunda ordem do operador diferencial correspondente, e, portanto, a métrica efetiva e a conexão efetiva são alteradas. De acordo com o primeiro método, o produto estrela como um todo é considerado um produto, i.e., como um operador de ordem zero. Isto garante a regularidade da expansão do kernel de calor $[139,140]$ para $\Theta$ pequeno. Para laplacianos $\mathrm{NC}$ mais gerais (contendo produtos estrela tanto à direita quanto esquerda) esta regularidade é perdida $[141,142]$. Entretanto, vamos considerar o operador de calor $h(t)=e^{-t\left(P_{0}+P_{2}\right)}$ em que $P_{0}$ não depende de $\theta$, enquanto $P_{2}$ é pelo menos bilinear no parâmetro fermiônico. Obviamente, $h(t)$ pode ser expandido numa série em $P_{2}$, e convergência não é uma questão, uma vez que a expansão é finita. Estes argumentos simples mostram que num caso mais geral o segundo método provavelmente funcionará, enquanto o primeiro provavelmente não o fará.

Coletando (4.13), (4.16) e (4.21), vemos que a parte divergente da ação efetiva é proporcional a $\hat{F}_{\mu \nu}^{2}$ e pode ser cancelada por uma renormalização do acoplamento $g$ na ação clássica (4.10). Logo, o modelo (4.10) com um espinor quantizado e um campo de fundo vetorial é renormalizável.

\subsection{Conclusões}

Nesse capítulo estudamos propriedades de renormalização de teorias $\mathrm{NC}$ em quatro dimensões com um parâmetro NC bifermiônico. Encontramos um modelo escalar renormalizável em todas as ordens da expansão em loops, dando assim mais um exemplo a uma família (não muito rica) de teorias NC 
não-supersimétricas renormalizáveis em quatro dimensões. Também descobrimos que esse modelo tem um ponto fixo infravermelho estável ao nível de um loop.

Também consideramos outro modelo, eletrodinâmica NC, que é renormalizável a um loop com parâmetro NC usual, e verificamos que a introdução de um parâmetro NC bifermiônico não destrói renormalizibilidade a um loop pelo menos no setor com pernas externas de fótons. Concluímos que não-comutatividade bifermiônica é amigável quanto à renormalização. Parece assim uma versão promissora de não-comutatividade, a ser levada à sério, e que exige mais estudo.

O primeiro problema a ser estudado se refere à interpretação física de não-comutatividade bifermiônica. Provavelmente, uma escolha com maior motivação física seria $\Theta^{\mu \nu} \propto \bar{\eta}\left[\gamma^{\mu}, \gamma^{\nu}\right] \eta$, em que $\eta$ é um espinor de Majorana anti-comutativo [129]. Então $\eta$ poderia ser interpretado como um campo espinorial cujas flutuações estão fixas por algum mecanismo. Para fazer valer essa interpretação, teríamos de considerar o caso em que $\eta$ não é constante, e consequentemente, o caso de não-comutatividade dependente do tempo. É evidente que em tal caso o produto estrela não teria uma expansão finita (cf. [144]), mas ainda assim a estrutura dessa expansão seria bastante mais simples que o caso geral. Esta talvez seja uma outra aplicação de não-comutatividade bifermiônica.

Para incorporar não-comutatividade bifermiônica no contexto de geometria não-comutativa, tem-se de encontrar uma álgebra $C^{*}$ correspondente. Esta tarefa é dificultada pela presença de dois tipos de produtos na álgebra e pelo segundo termo em (4.2), que não parece ser limitado na norma $L_{2}$. 


\section{Apêndice A}

\section{Ordenamento de Weyl de operadores e funções na álgebra de Berezin}

Vamos escrever o operador hamiltoniano (2.8) explicitamente:

$$
\hat{H}=-\lambda\left(\hat{p}^{2}+q t_{a \alpha \beta}\left(\hat{p}^{\mu} \hat{A}_{\mu}^{a}+\hat{A}_{\mu}^{a} \hat{p}^{\mu}\right) \hat{a}_{\alpha}^{\dagger} \hat{a}_{\beta}+q^{2} t_{a \alpha \beta} t_{b \gamma \delta} \hat{A}_{\mu}^{a} \hat{A}^{\mu b} \hat{a}_{\alpha}^{\dagger} \hat{a}_{\beta} \hat{a}_{\gamma}^{\dagger} \hat{a}_{\delta}-m^{2}\right) .
$$

Simetrização total em $\hat{x}$ e $\hat{p}$, e anti-simetrização total em $a^{\dagger}$ e $a$ dá o operador hamiltoniano ordenado $\hat{H}_{W}$ :

$$
\hat{H}_{W}=-\lambda\left(\hat{p}^{2}+\frac{q}{2} t_{a \alpha \beta}\left(\hat{p}^{\mu} \hat{A}_{\mu}^{a}+\hat{A}_{\mu}^{a} \hat{p}^{\mu}\right)\left[\hat{a}_{\alpha}^{\dagger}, \hat{a}_{\beta}\right]+q^{2} t_{a \alpha \beta} t_{b \gamma \delta} \hat{A}_{\mu}^{a} \hat{A}^{\mu b}\left(\hat{a}_{\alpha}^{\dagger} \hat{a}_{\beta} \hat{a}_{\gamma}^{\dagger} \hat{a}_{\delta}\right)_{W}-m^{2}\right),
$$

onde o termo com quatro férmions é dado por

$\hat{a}_{\alpha}^{\dagger} \hat{a}_{\beta} \hat{a}_{\gamma}^{\dagger} \hat{a}_{\delta}=\left(\hat{a}_{\alpha}^{\dagger} \hat{a}_{\beta} \hat{a}_{\gamma}^{\dagger} \hat{a}_{\delta}\right)_{W}+\frac{1}{2} \delta_{\gamma \delta}\left(\hat{a}_{\alpha}^{\dagger} \hat{a}_{\beta}\right)_{W}-\frac{1}{2} \delta_{\delta \alpha}\left(\hat{a}_{\gamma}^{\dagger} \hat{a}_{\beta}\right)_{W}+\delta_{\alpha \beta}\left(\hat{a}_{\gamma}^{\dagger} \hat{a}_{\delta}\right)_{W}+\frac{1}{2} \delta_{\gamma \beta}\left(\hat{a}_{\alpha}^{\dagger} \hat{a}_{\delta}\right)_{W}-\frac{1}{4} \delta_{\delta \alpha} \delta_{\gamma \beta}-\delta_{\alpha \beta} \delta_{\gamma \delta}$

Usando o fato de que as matrizes $t_{a}$ têm traço nulo, e a anti-simetria das constantes de estrutura $f_{a b c}$, temos

$$
\hat{H}=\hat{H}_{W}+\lambda \frac{q^{2}}{4} \operatorname{tr}\left(t_{a} t_{b}\right) \hat{A}_{\mu}^{a} \hat{A}^{\mu b}
$$

Logo, o hamiltoniano é a soma de uma expressão ordenada segundo Weyl mais uma contribuição que não é invariante de calibre. O símbolo de Weyl correspondente a $\hat{H}_{W}$ é

$$
H_{W}=-\lambda\left(p^{2}+2 q t_{a \alpha \beta}\left(p^{\mu} A_{\mu}^{a}\right) \bar{\chi}_{\alpha} \chi_{\beta}+q^{2} t_{a \alpha \beta} t_{b \gamma \delta} A_{\mu}^{a} A^{\mu b} \bar{\chi}_{\alpha} \chi_{\beta} \bar{\chi}_{\gamma} \chi_{\delta}-m^{2}\right)
$$

\section{Demonstração $^{1}$ da regra do ponto médio bifermiônico}

Seja $F\left(\hat{a}, \hat{a}^{\dagger}\right)$ qualquer polinômio em $\mathrm{e}^{\dagger}$ ordenado segundo Weyl, então

$$
\begin{aligned}
\left\langle\bar{\chi}\left|F\left(\hat{a}, \hat{a}^{\dagger}\right)\right| \chi\right\rangle & =\int d \bar{\eta} d \eta\langle\bar{\chi} \mid \eta\rangle F\left(\frac{\chi+\eta}{2}, \bar{\eta}\right)\langle\bar{\eta} \mid \chi\rangle, \\
& =\int d \bar{\eta} d \eta\langle\bar{\chi} \mid \eta\rangle F\left(\chi, \frac{\bar{\chi}+\bar{\eta}}{2}\right)\langle\bar{\eta} \mid \chi\rangle,
\end{aligned}
$$

\footnotetext{
${ }^{1}$ Adaptado de [145]
} 
Vamos provar a identidade (A.3). A demonstração da segunda identidade é análoga. Primeiro, considere $F\left(\hat{a}^{\dagger}\right)$ um polinômio em operadores de criação. Claramente, $F$ está ordenado segundo Weyl, e (A.3) é trivialmente satisfeita,

$$
\left\langle\bar{\chi}\left|F\left(\hat{a}^{\dagger}\right)\right| \chi\right\rangle=\int d \bar{\eta} d \eta\langle\bar{\chi} \mid \eta\rangle F(\bar{\chi})\langle\bar{\eta} \mid \chi\rangle .
$$

Agora, para $F\left(\hat{a}, \hat{a}^{\dagger}\right)=\frac{1}{2}\left(\hat{a}_{\alpha} f\left(\hat{a}^{\dagger}\right)+(-1)^{\varepsilon(f)} f\left(\hat{a}^{\dagger}\right) \hat{a}_{\alpha}\right)$, em que o sinal depende da paridade de $f\left(\hat{a}^{\dagger}\right),($ A.3) é evidentemente satisfeita. Qualquer polinômio com ordenamento de Weyl pode ser obtido a partir de repetidas anti-simetrização da forma $F=\frac{1}{2}\left(\hat{a}_{\alpha} f \pm f \hat{a}_{\alpha}\right)$ em que $f\left(\hat{a}, \hat{a}^{\dagger}\right)$ está ordenado. Logo, vamos provar (A.3) indutivamente, assumindo que vale para $f\left(\hat{a}, \hat{a}^{\dagger}\right)$ e provar que vale também para $F=\frac{1}{2}\left(\hat{a}_{\alpha} f \pm f \hat{a}_{\alpha}\right)$,

$$
\begin{aligned}
\left\langle\bar{\chi}\left|\frac{1}{2}\left(\hat{a}_{\alpha} f \pm f \hat{a}_{\alpha}\right)\right| \chi\right\rangle & =\int d \bar{\eta} d \eta \frac{1}{2}\left(\left\langle\bar{\chi}\left|\hat{a}_{\alpha}\right| \eta\right\rangle\langle\bar{\eta}|f| \chi\rangle \pm\langle\bar{\chi} \mid \eta\rangle\left\langle\bar{\eta}\left|f \hat{a}_{\alpha}\right| \chi\right\rangle\right) \\
& =\int d \bar{\eta} d \eta\langle\bar{\chi} \mid \eta\rangle \frac{\eta_{\alpha}+\chi_{\alpha}}{2}\langle\bar{\eta}|f| \chi\rangle \\
& =\int d \bar{\eta} d \eta d \bar{\xi} d \xi\langle\bar{\chi} \mid \eta\rangle\langle\bar{\eta} \mid \xi\rangle \frac{\chi_{\alpha}+\eta_{\alpha}}{2} f\left(\frac{\chi+\xi}{2}, \bar{\xi}\right)\langle\bar{\xi} \mid \chi\rangle \\
& =d \bar{\xi} d \xi\langle\bar{\chi} \mid \xi\rangle \frac{\chi_{\alpha}+\xi_{\alpha}}{2} f\left(\frac{\chi+\xi}{2}, \bar{\xi}\right)\langle\bar{\xi} \mid \chi\rangle
\end{aligned}
$$

em que na última igualdade usamos a identidade

$$
\int d \bar{\eta} d \eta\langle\bar{\alpha} \mid \eta\rangle\langle\bar{\eta} \mid \beta\rangle f(\eta)=\langle\bar{\alpha} \mid \beta\rangle f(\beta) .
$$




\section{Apêndice B}

\section{Notação e identidades úteis ao capítulo 2}

Nossa convenção de sinais foi tirada de [111]. Usamos o tensor $\eta^{a b}=\eta_{a b}=\operatorname{diag}(+1,-1)$ para subir e descer índices. O tensor de Levi-Civita é definido por $\varepsilon^{01}=-1$, tal que valem as seguintes relações

$$
\varepsilon^{10}=\varepsilon_{01}=1, \quad \varepsilon^{0}{ }_{1}=\varepsilon^{1}{ }_{0}=-\varepsilon_{0}{ }^{1}=-\varepsilon_{1}{ }^{0}=1 .
$$

Estas relações são válidas tanto para $\varepsilon^{a b}$ quanto para $\varepsilon^{\mu \nu}$. Note que $\varepsilon^{\mu \nu}$ é sempre usado com os dois índices em cima.

Nossa convenção para o comutador $[\cdot, \cdot]$ é dependente do contexto. No plano comutativo, com a álgebra de funções usual dada pelo produto pontual entre funções, o comutador entre uma $p$-forma $P$ que toma valores numa álgebra de Lie $\mathfrak{g}$ e uma $q$-forma $Q$ que toma valores em $\mathfrak{g}$ é

$$
[P, Q]=P \wedge Q-(-1)^{p q} Q \wedge P=P^{i} \wedge Q^{j}\left[\tau_{i}, \tau_{j}\right],
$$

onde $\tau_{i}$ é uma base para a álgebra. Ademais, a derivada covariante é dada pela expressão

$$
D=d+[A, \cdot] .
$$

No plano não-comutativo, com produto de funções deformado pelo produto de Moyal, o comutador de uma $p$-forma $P$ que toma valores em uma álgebra de Lie $\mathfrak{g}$ com uma $q$-forma $Q$ que toma valores em $\mathfrak{g}$ é dado pela expressão análoga

$$
[P, Q]=P \wedge^{\star} Q-(-1)^{p q} Q \wedge^{\star} P
$$

em que o produto $\wedge^{\star}$ tem uma definição semelhante à definição do produto exterior, dada por:

$$
\left(P \wedge^{\star} Q\right)\left(\partial_{\mu_{1}}, \partial_{\mu_{2}}\right)=\frac{1}{2}\left(P_{\mu_{1}} \star Q_{\mu_{2}}-P_{\mu_{2}} \star Q_{\mu_{1}}\right),
$$

em que $P_{\mu}$ e $Q_{\mu}$ são as componentes das 1-formas $P$ e $Q$ na base holonômica $\left\{\partial_{0}, \partial_{1}\right\}$. Usando a propriedade de fechamento (C.3) do produto estrela, é imediato ver que o produto $\wedge^{\star}$ possui uma propriedade de simetria bem definida por troca de fatores sob o sinal de integração,

$$
\int_{M} P \wedge^{\star} Q=-\int_{M} Q \wedge^{\star} P
$$


Usando a propriedade de simetria sob o signo de integração, o comutador estrela também pode ser escrito como

$$
\int_{M}[P, Q]=\int_{M} P^{A} \wedge^{*} Q^{B}\left[\tau_{A}, \tau_{B}\right]
$$

em que $\tau_{A}$ é uma base para a álgebra. Nesse contexto, a derivada covariante é dada por uma expressão formalmente idêntica ao caso comutativo:

$$
D=d+[A, \cdot] .
$$

Em particular, para $P$ uma 1-forma com valores em uma álgebra de Lie, temos

$$
\begin{aligned}
D P\left(\partial_{\mu}, \partial_{\nu}\right) & =d P\left(\partial_{\mu}, \partial_{\nu}\right)+A \wedge^{\star} P\left(\partial_{\mu}, \partial_{\nu}\right)+P \wedge^{\star} A\left(\partial_{\mu}, \partial_{\nu}\right) \\
& =\frac{1}{2}\left(\partial_{\mu} P_{\nu}^{A}-\partial_{\nu} P_{\mu}^{A}\right) \tau_{A}+\frac{1}{2}\left(A_{\mu}^{A} \star P_{\nu}^{B}-A_{\nu}^{A} \star P_{\mu}^{B}\right) \tau_{A} \tau_{B}+\frac{1}{2}\left(P_{\mu}^{A} \star A_{\nu}^{B}-P_{\nu}^{A} \star A_{\mu}^{B}\right) \tau_{A} \tau_{B} .
\end{aligned}
$$

Em se tratando do comutador entre formas ou funções reais, ele assume a expressão usual, não graduada,

$$
\begin{aligned}
& {[P, Q]=P \wedge^{\star} Q-Q \wedge^{\star} P} \\
& {\left[P_{\mu}, Q_{\nu}\right]=P_{\mu} \star Q_{\nu}-Q_{\nu} \star P_{\mu}} \\
& {\left[P_{\mu}, Q_{\nu}\right]_{+}=P_{\mu} \star Q_{\nu}+Q_{\nu} \star P_{\mu}}
\end{aligned}
$$

As seguintes identidades úteis valem para funções arbitrárias $A_{1}, A_{2}, B_{1}$ e $B_{2}$ :

$$
\begin{aligned}
& \int\left(\left[A_{1}, B_{1}\right] \star\left[B_{2}, A_{2}\right]-\left[B_{1}, A_{2}\right] \star\left[A_{1}, B_{2}\right]\right)= \\
& =-\int\left[A_{1}, A_{2}\right] \star\left[B_{1}, B_{2}\right] \\
& \int\left(\left[A_{1}, B_{1}\right]_{+} \star\left[A_{2}, B_{2}\right]_{+}-\left[A_{1}, B_{2}\right]_{+} \star\left[A_{2}, B_{1}\right]_{+}\right)= \\
& =-\int\left[A_{1}, A_{2}\right] \star\left[B_{1}, B_{2}\right] \\
& \int\left(\left[A_{1}, B_{1}\right]_{+} \star\left[B_{2}, A_{2}\right]-\left[B_{1}, A_{2}\right]_{+} \star\left[A_{1}, B_{2}\right]\right)= \\
& =\int\left[B_{1}, B_{2}\right] \star\left[A_{1}, A_{2}\right]_{+} \\
& \int\left(\left[A_{1}, B_{1}\right] \star\left[A_{2}, B_{2}\right]-\left[A_{1}, B_{2}\right]_{+} \star\left[A_{2}, B_{1}\right]_{+}\right)= \\
& =-\int\left[A_{1}, A_{2}\right]_{+} \star\left[B_{1}, B_{2}\right]_{+}
\end{aligned}
$$

Por meio da fórmula

$$
\varepsilon_{a b} \varepsilon_{c d}=\eta_{b c} \eta_{a d}-\eta_{a c} \eta_{b d}
$$

pode-se eliminar símbolos $\varepsilon$ repetidos.

O grupo $U(1,1)$ é o grupo de matrizes complexas de ordem 2 que preservam a forma quadrática em $\mathbb{C}^{2}$

$$
\left|z_{1}\right|^{2}-\left|z_{2}\right|^{2}=c t e
$$


A álgebra de Lie $s u(1,1)$ é dada por matrizes da forma

$$
\left(\begin{array}{cc}
z_{1} & w \\
\bar{w} & z_{2}
\end{array}\right)
$$

em que $\bar{z}_{1}=-z_{1}, \bar{z}_{2}=-z_{2}, z_{1}+z_{2}=0$ e $w$ arbitrário. Uma base para $s u(1,1)$ é

$$
\tau_{0}=\frac{1}{2}\left(\begin{array}{cc}
i & 0 \\
0 & -i
\end{array}\right), \tau_{1}=\frac{1}{2}\left(\begin{array}{cc}
0 & 1 \\
1 & 0
\end{array}\right), \tau_{2}=\frac{1}{2}\left(\begin{array}{cc}
0 & -i \\
i & 0
\end{array}\right)
$$

Os elementos da base acima satisfazem as relações de comutação

$$
\left[\tau_{i}, \tau_{j}\right]=-\varepsilon_{i j}{ }^{k} \tau_{k}, i=0,1,2,
$$

comum às álgebras $s o(2,1)$ e $s l(2, R)$. A forma de Killing da álgebra $s u(1,1)$ é

$$
\eta_{i j}=\frac{1}{2} \varepsilon_{i k}{ }^{l} \varepsilon_{j l}^{k}=\operatorname{diag}(-1,1,1) .
$$

Para formar uma base de $u(1,1)$ basta adicionar um elemento com traço diferente de zero e com diagonais puramente imaginárias, por exemplo,

$$
\tau_{3}=\frac{1}{2}\left(\begin{array}{cc}
i & 0 \\
0 & i
\end{array}\right)
$$

Evidentemente,

$$
\left[\tau_{3}, \tau_{i}\right]=0 .
$$

A base $\tau_{A}, A=0,1,2,3$, de $u(1,1)$ está normalizada a $\operatorname{tr} \tau_{A} \tau_{B}=\frac{1}{2} \eta_{A B}, \eta_{A B}=\operatorname{diag}(-1,1,1,-1)$. Nessa base valem as relações

$$
\begin{aligned}
& \tau_{i} \tau_{j}=-\frac{1}{2} \epsilon_{i j k} \tau^{k}-\frac{i}{2} \eta_{i j} \tau_{3} \\
& \tau_{i} \tau_{3}=\frac{i}{2} \tau_{i} \\
& \tau_{3}^{2}=\frac{i}{2} \tau_{3}
\end{aligned}
$$




\section{Apêndice C}

\section{Produto estrela de Moyal}

A não-comutatividade do espaço-tempo é usualmente descrita por meio de uma álgebra $C^{*}$ de operadores gerada por operadores $\hat{x}$ que satisfazem

$$
\left[\hat{x}^{i}, \hat{x}^{j}\right]=i \theta^{i j}
$$

em que $\theta$ é uma matriz anti-simétrica constante. Um modo de implementar essa não-comutatividade do espaço-tempo é através do produto estrela de Moyal, uma deformação da álgebra de funções sobre o $\mathbb{R}^{D}$ induzida pela correspondência de Weyl $W$, que associa operadores $\hat{f}: L^{2}\left(\mathbb{R}^{D}\right) \rightarrow L^{2}\left(\mathbb{R}^{D}\right)$ a seus símbolos $f: \mathbb{R}^{D} \rightarrow \mathbb{C}$,

$$
\hat{f}=W(f)=\int d^{D} \eta \tilde{f}(\eta) e^{i \eta \cdot \hat{x}},
$$

em que $\tilde{f}(\eta)$ é a transformada de Fourrier de $f(x)$,

$$
\tilde{f}(\eta)=\int \frac{d^{D} x}{(2 \pi)^{D}} f(x) e^{-i \eta \cdot x}
$$

Para $f$ real, $W(f)$ é hermitiano e no limite comutativo, $\theta \rightarrow 0$, tem-se simplesmente $\hat{f}=f(\hat{x})$. Usando a correspondência de Weyl e a fórmula de Baker-Campbell-Hausdorf,

$$
e^{-i \eta \cdot \hat{x}} e^{-i \xi \cdot \hat{x}}=e^{-\frac{i}{2} \theta^{i j} \eta_{i} \xi_{j}} e^{-i(\eta+\xi) \cdot \hat{x}},
$$

calcula-se o símbolo correspondente ao produto $\hat{f} \hat{g}$ :

$$
\begin{aligned}
W(f) W(g) & =\int d^{D} \xi\left[\int d^{D} \eta \tilde{f}(\eta) \tilde{g}(\xi-\eta) e^{-\frac{i}{2} \theta^{i j} \eta_{i} \xi_{j}}\right] e^{i \xi \cdot \hat{x}} \\
& =\int d^{D} \xi \widetilde{f \star g}(\xi) e^{i \xi \cdot \hat{x}}=W(f \star g)
\end{aligned}
$$

onde

$$
\widetilde{f \star g}(\xi)=\int d^{D} \eta \tilde{f}(\eta) \tilde{g}(\xi-\eta) e^{-\frac{i}{2} \theta^{i j} \eta_{i} \xi_{j}} .
$$


Logo, o símbolo de $\hat{f} \hat{g}$ é

$$
\begin{aligned}
f \star g(x) & =\int d^{D} \xi e^{i \xi \cdot x} \widetilde{f \star g}(\xi)=\int d^{D} \xi d^{D} \eta \tilde{f}(\eta) \tilde{g}(\xi-\eta) e^{-\frac{i}{2} \theta^{i j} \eta_{i} \xi_{j}} e^{i \xi \cdot x} \\
& =\left.\exp \left(\frac{i}{2} \theta^{i j} \frac{\partial}{\partial \xi^{i}} \frac{\partial}{\partial \eta^{j}}\right) f(x+\xi) g(x+\eta)\right|_{\xi=\zeta=0} \\
& =f(x) g(x)+\sum_{n=1}^{\infty}\left(\frac{i}{2}\right)^{n} \frac{1}{n !} \theta^{i_{1} j_{1}} \cdots \theta^{i_{n} j_{n}} \partial_{i_{1}} \cdots \partial_{i_{n}} f(x) \theta^{i_{1} j_{1}} \cdots \theta^{i_{n} j_{n}} \partial_{j_{1}} \cdots \partial_{j_{n}} g((\mathbb{C}, 1)
\end{aligned}
$$

Esse produto é associativo, $(f \star g) \star h=f \star(g \star h)$ e para caso em que $f=x^{i}$ e $g=x^{j}$ recuperamos as relações de comutação básicas

$$
x^{i} \star x^{j}-x^{j} \star x^{i}=i \theta^{i j} .
$$

Ademais, o produto de Moyal é fechado, i.e, o produto estrela de duas funções integráveis é integrável,

$$
\int_{\mathcal{M}} d^{2} x f \star g=\int_{\mathcal{M}} d^{2} x f g(x)
$$

e respeita a regra de Leibniz

$$
\partial_{i}(f \star g)=\left(\partial_{i} f\right) \star g+f \star\left(\partial_{i} g\right),
$$

e permite que se façam permutações cíclicas sob o sinal de integração

$$
\int_{\mathcal{M}} d^{2} x f \star g \star h=\int_{\mathcal{M}} d^{2} x h \star f \star g .
$$

A conjugação complexa inverte a ordem dos fatores,

$$
(f \star g)^{*}=g^{*} \star f^{*} .
$$

O produto (C.1) não é a única escolha possível de um produto associativo e não-comutativo. $\mathrm{O}$ lado direito de (C.2) pode depender, em princípio, das coordenadas. 


\section{Apêndice D}

\section{Simetria BRST e eletrodinâmica não-comutativa}

A ação clássica para a eletrodinâmica no $\mathbb{R}^{4}$ não-comutativo é

$$
\begin{aligned}
& S_{c l}=\int d^{4} x \bar{\psi}\left(i \gamma^{\mu} D_{\mu}-m\right) \psi-\frac{1}{4 g^{2}} \int \hat{F}_{\mu \nu} \star \hat{F}^{\mu \nu}, \\
& \quad D_{\mu} \psi=\partial_{\mu} \psi-i A_{\mu} \star \psi, \hat{F}_{\mu \nu}=\partial_{\mu} A_{\nu}-\partial_{\nu} A_{\mu}-i\left[A_{\mu}, A_{\nu}\right],
\end{aligned}
$$

em que $A_{\mu}$ é a conexão de calibre real, $\psi$ é um espinor de Dirac e (anti)comutadores são calculados com produto estrela. Sob as transformações

$$
\begin{aligned}
& A_{\mu} \mapsto \omega \star A_{\mu} \star \omega^{-1}-i \partial_{\mu} \omega \star \omega^{-1}, \\
& \psi \mapsto \omega \star \psi, \bar{\psi} \mapsto \bar{\psi} \star \omega^{-1}, \omega=e^{i \lambda(x)}
\end{aligned}
$$

tem-se

$$
D_{\mu} \psi \mapsto \omega \star D_{\mu} \psi, F_{\mu \nu} \mapsto \omega \star F_{\mu \nu} \star \omega^{-1},
$$

de tal forma que $S_{c l}$ é invariante por transformações do grupo $U_{\star}(1)$.

Agora considere o espaço funcional estendido dos campos fantasmas $c$ e $\bar{c}$, e a transformação BRST

$$
s A_{\mu}=D_{\mu} c=\partial_{\mu} c-i\left[A_{\mu}, c\right] .
$$

A condição $s^{2} A_{\mu}=0$ implica

$$
\begin{aligned}
s^{2} A_{\mu} & =\partial_{\mu} s c-i\left[s A_{\mu}, c\right]_{+}-i\left[A_{\mu}, s c\right] \\
& =D_{\mu}(s c)-i\left[D_{\mu} c, c\right]_{+}=D_{\mu}(s c)-i D_{\mu}(c \star c)=0
\end{aligned}
$$

Logo, a transformação do campo fantasma $c$ tem de ser

$$
s c=i c \star c, s^{2} c=0 .
$$

Para o campo anti-fantasma $\bar{c}$, postulamos a transformação usual

$$
s \bar{c}=i B, s B=0 .
$$


A ação de Maxwell é invariante com respeito à transformação BRST do campo de calibre $A_{\mu}$,

$$
\begin{aligned}
s \int d^{4} x \hat{F}_{\mu \nu} \star \hat{F}^{\mu \nu} & =2 \int d^{4} x\left(s \hat{F}_{\mu \nu}\right) \star \hat{F}^{\mu \nu}=-i \int d^{4} x\left[\hat{F}_{\mu \nu}, c\right] \star \hat{F}^{\mu \nu} \\
& =-i \int d^{4} x\left(\hat{F}_{\mu \nu} \star c \star \hat{F}^{\mu \nu}-c \star \hat{F}_{\mu \nu} \star \hat{F}^{\mu \nu}\right)=0 .
\end{aligned}
$$

Agora, para encontrar a transformação BRST dos campos de matéria, consideramos primeiro a transformação da parte cinética da densidade lagrangiana de matéria,

$$
\begin{aligned}
s\left(i \bar{\psi} \gamma^{\mu} D_{\mu} \psi\right) & =i s\left(\bar{\psi} \gamma^{\mu} \star \partial_{\mu} \psi\right)+s\left(\bar{\psi} \gamma^{\mu} \star A_{\mu} \star \psi\right) \\
& =i s\left(\bar{\psi} \gamma^{\mu} \star \partial_{\mu} \psi\right)-\bar{\psi} \gamma^{\mu} \star s A_{\mu} \star \psi+s \bar{\psi} \gamma^{\mu} \star A_{\mu} \star \psi-\bar{\psi} \gamma^{\mu} \star A_{\mu} \star s \psi
\end{aligned}
$$

Em seguida, exige-se que todos os termos contendo derivadas dos campos se cancelem,

$$
i s \bar{\psi} \gamma^{\mu} \star \partial_{\mu} \psi-i \bar{\psi} \gamma^{\mu} \star \partial_{\mu} s \psi-\bar{\psi} \gamma^{\mu} \star \partial_{\mu} c \star \psi=0 .
$$

Isto pode ser alcançado se $\psi$ e $\bar{\psi}$ se transformarem como

$$
s \psi=i c \star \psi, s \bar{\psi}=i \bar{\psi} \star c, s^{2} \psi=s^{2} \bar{\psi}=0 .
$$

De fato, com as transformações acima, todos os outros termos também se anulam, de modo que a densidade lagrangiana de matéria é invariante. Logo, a ação da eletrodinâmica não-comutativa é invariante pelas transformações BRST

$$
\begin{aligned}
& s A_{\mu}=\partial_{\mu} c-i\left[A_{\mu}, c\right], s c=i c \star c, s \bar{c}=i B, s B=0, \\
& s \psi=i c \star \psi, s \bar{\psi}=i \bar{\psi} \star c .
\end{aligned}
$$

A matriz $S$ e o termo de fixação de calibre podem ser escritos como

$$
\begin{aligned}
& S=N^{-1} \int \exp i\left(S_{c l}+S_{G F}\right) d x D A D \bar{\psi} D \psi D \bar{c} D c D B \\
& S_{G F}=\int d^{4} x s\left[\bar{c} \star\left(\frac{\alpha}{2} B+\partial_{\mu} A^{\mu}\right)\right] .
\end{aligned}
$$

Integrando $B$, simplifica-se a integral de trajetória para

$$
S=N^{-1} \int \exp i\left[S_{c l}+\int\left(-\frac{1}{2 \alpha}(\partial \cdot A)^{2}-i \partial^{\mu} \bar{c} \star D_{\mu} c\right) d x\right] D A D \bar{\psi} D \psi D \bar{c} D c
$$

que é a representação usual da integral de trajetória para a matriz $S$ envolvendo um termo de fixação de calibre de Lorentz e o determinante de Faddeev-Popov. 


\section{Referências Bibliográficas}

[1] Hartland S. Snyder. Quantized space-time. Phys. Rev., 71:38-41, 1947.

[2] Alain Connes. Non-commutative differential geometry. Inst. Hautes Études, 62(2):41-144, 1985.

[3] S. L. Woronowicz. Twisted SU(2) group: An Example of a noncommutative differential calculus. Publ. Res. Inst. Math. Sci. Kyoto, 23:117-181, 1987.

[4] John von Neumann. Mathematical Foundations of Quantum Mechanics. Princeton landmarks in mathematics and physics series. 1955. Translated from the German edition by Robert T. Beyer. Original first edition published in German in 1932.

[5] A. Connes and Marc A. Rieffel. Yang-Mills for noncommutative two-tori. Contemp. Math., 62:237-266, 1987.

[6] Ali H. Chamseddine, Giovanni Felder, and J. Frohlich. Gravity in noncommutative geometry. Commun. Math. Phys., 155:205-218, 1993.

[7] W. Kalau and M. Walze. Gravity, noncommutative geometry and the Wodzicki residue. J. Geom. Phys., 16:327-344, 1995.

[8] Daniel Kastler. The Dirac operator and gravitation. Commun. Math. Phys., 166:633-644, 1995.

[9] Ali H. Chamseddine and Alain Connes. The spectral action principle. Commun. Math. Phys., 186:731-750, 1997.

[10] Alain Connes and John Lott. PARTICLE MODELS AND NONCOMMUTATIVE GEOMETRY (EXPANDED VERSION). Nucl. Phys. Proc. Suppl., 18B:29-47, 1991.

[11] Joseph C. Varilly and Jose M. Gracia-Bondia. Connes' noncommutative differential geometry and the Standard Model. J. Geom. Phys., 12:223-301, 1993.

[12] C. P. Martin, Jose M. Gracia-Bondia, and Joseph C. Varilly. The standard model as a noncommutative geometry: The low- energy regime. Phys. Rept., 294:363-406, 1998.

[13] J. Bellissard, A. van Elst, and H. Schulz-Baldes. The noncommutative geometry of the quantum hall effect. J. Math. Phys., 35:5373, 1994. 
[14] Shiraz Minwalla, Mark Van Raamsdonk, and Nathan Seiberg. Noncommutative perturbative dynamics. JHEP, 02:020, 2000.

[15] I. Ya. Aref'eva, D. M. Belov, and A. S. Koshelev. Two-loop diagrams in noncommutative phi**4(4) theory. Phys. Lett., B476:431-436, 2000.

[16] Iouri Chepelev and Radu Roiban. Convergence theorem for non-commutative feynman graphs and renormalization. JHEP, 03:001, 2001.

[17] Harald Grosse and Raimar Wulkenhaar. Power-counting theorem for non-local matrix models and renormalisation. Commun. Math. Phys., 254:91-127, 2005.

[18] Harald Grosse and Raimar Wulkenhaar. Renormalisation of phi**4 theory on noncommutative $\mathrm{R}^{* *} 2$ in the matrix base. JHEP, 12:019, 2003.

[19] Harald Grosse and Raimar Wulkenhaar. Renormalisation of phi**4 theory on noncommutative r**4 in the matrix base. Commun. Math. Phys., 256:305-374, 2005.

[20] Edwin Langmann and Richard J. Szabo. Duality in scalar field theory on noncommutative phase spaces. Phys. Lett., B533:168-177, 2002.

[21] Razvan Gurau, Jacques Magnen, Vincent Rivasseau, and Fabien Vignes-Tourneret. Renormalization of non-commutative phi**4(4) field theory in x space. Commun. Math. Phys., $267: 515-542,2006$.

[22] Fabien Vignes-Tourneret. Renormalization of the orientable non-commutative Gross- Neveu model. Annales Henri Poincare, 8:427-474, 2007.

[23] Harald Grosse and Harold Steinacker. Renormalization of the noncommutative phi** 3 model through the Kontsevich model. Nucl. Phys., B746:202-226, 2006.

[24] Harald Grosse and Harold Steinacker. A nontrivial solvable noncommutative phi**3 model in 4 dimensions. JHEP, 08:008, 2006.

[25] Harald Grosse and Harold Steinacker. Exact renormalization of a noncommutative phi**3 model in 6 dimensions. 2006.

[26] Edward Witten. Noncommutative Geometry and String Field Theory. Nucl. Phys., B268:253, 1986.

[27] Amit Giveon, Massimo Porrati, and Eliezer Rabinovici. Target space duality in string theory. Phys. Rept., 244:77-202, 1994.

[28] Edward Witten. Bound states of strings and p-branes. Nucl. Phys., B460:335-350, 1996.

[29] D. Amati, M. Ciafaloni, and G. Veneziano. Can Space-Time Be Probed Below the String Size? Phys. Lett., B216:41, 1989.

[30] G. Veneziano. A Stringy Nature Needs Just Two Constants. Europhys. Lett., 2:199, 1986.

[31] David J. Gross and Paul F. Mende. String Theory Beyond the Planck Scale. Nucl. Phys., B303:407, 1988. 
[32] Michael R. Douglas and Christopher M. Hull. D-branes and the noncommutative torus. JHEP, 02:008, 1998.

[33] Chong-Sun Chu and Pei-Ming Ho. Noncommutative open string and d-brane. Nucl. Phys., B550:151-168, 1999.

[34] Nathan Seiberg and Edward Witten. String theory and noncommutative geometry. JHEP, 09:032, 1999.

[35] Volker Schomerus. D-branes and deformation quantization. JHEP, 06:030, 1999.

[36] Daniela Bigatti and Leonard Susskind. Magnetic fields, branes and noncommutative geometry. Phys. Rev., D62:066004, 2000.

[37] Richard J. Szabo. Quantum field theory on noncommutative spaces. Phys. Rept., 378:207-299, 2003.

[38] Carlo Rovelli. Quantum gravity. Cambridge, UK: Univ. Pr. (2004) 455 p.

[39] F. A. Berezin and M. S. Marinov. Particle spin dynamics as the grassmann variant of classical mechanics. Ann. Phys., 104:336, 1977.

[40] M. Gadella. Moyal formulation of quantum mechanics. Fortschr. Phys., 43:229-, 1995.

[41] S. P. Gavrilov and D. M. Gitman. Quantization of point-like particles and consistent relativistic quantum mechanics. Int. J. Mod. Phys., A15:4499-4538, 2000.

[42] R. Fresneda, S. P. Gavrilov, D. M. Gitman, and P. Yu. Moshin. Quantization of $(2+1)-$ spinning particles and bifermionic constraint problem. Class. Quant. Grav., 21:1419-1442, 2004 .

[43] A. P. Balachandran, Per Salomonson, Bo-Sture Skagerstam, and Jan-Olof Winnberg. Classical description of particle interacting with nonabelian gauge field. Phys. Rev., D15:2308, 1977.

[44] V. Ya. Fainberg and A. V. Marshakov. Local supersymmetry and dirac particle propagator as a path integral. Nucl. Phys., B306:659, 1988.

[45] E. S. Fradkin and D. M. Gitman. Path integral representation for the relativistic particle propagators and bfv quantization. Phys. Rev., D44:3230-3236, 1991.

[46] Dimitri M. Gitman. Path integrals and pseudoclassical description for spinning particles in arbitrary dimensions. Nucl. Phys., B488:490-512, 1997.

[47] D. M. Gitman and Sh. M. Shvartsman. Spinor and isospinor structure of relativistic particle propagators. Phys. Lett., B318:122-126, 1993.

[48] N. v. Borisov and P. p. Kulish. Path integral in superspace for a relativistic spinor particle in an external gauge field. Theor. Math. Phys., 51:535-540, 1982.

[49] F. A. Berezin and M. A. Shubin. The Schr?dinger Equation. Kluwer Academic Publishers, Dordrecht, 1991. 
[50] Anais Smailagic and Euro Spallucci. Isotropic representation of the noncommutative 2D harmonic oscillator. Phys. Rev., D65:107701, 2002.

[51] Anais Smailagic and Euro Spallucci. Noncommutative 3D harmonic oscillator. J. Phys., A35:L363-L368, 2002.

[52] Denis Kochan and Michal Demetrian. QM on non-commutative plane. 2001.

[53] M. Chaichian, M. M. Sheikh-Jabbari, and A. Tureanu. Hydrogen atom spectrum and the Lamb shift in noncommutative QED. Phys. Rev. Lett., 86:2716, 2001.

[54] J. Gamboa, M. Loewe, and J. C. Rojas. Non-Commutative Quantum Mechanics. Phys. Rev., D64:067901, 2001.

[55] M. Chaichian, A. Demichev, P. Presnajder, M. M. Sheikh-Jabbari, and A. Tureanu. Quantum theories on noncommutative spaces with nontrivial topology: Aharonov-Bohm and Casimir effects. Nucl. Phys., B611:383-402, 2001.

[56] M. Chaichian, A. Demichev, P. Presnajder, M. M. Sheikh-Jabbari, and A. Tureanu. AharonovBohm effect in noncommutative spaces. Phys. Lett., B527:149-154, 2002.

[57] Gianpiero Mangano. Path integral approach to noncommutative spacetimes. J. Math. Phys., 39:2584-2591, 1998.

[58] Ciprian Acatrinei. Path Integral Formulation of Noncommutative Quantum Mechanics. JHEP, 09:007, 2001.

[59] Branko Dragovich and Zoran Rakic. Path Integrals in Noncommutative Quantum Mechanics. Theor. Math. Phys., 140:1299-1308, 2004.

[60] Anais Smailagic and Euro Spallucci. Feynman path integral on the noncommutative plane. J. Phys., A36:L467, 2003.

[61] H. S. Tan. A coherent-state-based path integral for quantum mechanics on the Moyal plane. J. Phys., A39:15299-15310, 2006.

[62] D. M. Gitman and V. G. Kupriyanov. Path integral representations in noncommutative quantum mechanics and noncommutative version of Berezin-Marinov action. Eur. Phys. J., C54:325-332, 2008.

[63] C. Duval and P. A. Horvathy. The "Peierls substitution"and the exotic Galilei group. Phys. Lett., B479:284-290, 2000.

[64] A. A. Deriglazov. Noncommutative relativistic particle on the electromagnetic background. Phys. Lett., B555:83-88, 2003.

[65] A. A. Deriglazov. Noncommutative relativistic particle. 2002.

[66] Lebedev Phys. Inst. Quantum Electrodynamics of Phenomena in Intense Fields, 111. Nauka Moscow, 1979.

A. A. Grib, S.G. Mamaev, and V.M. Mostepanenko. Vacuum Quantum Effects in Strong Fields. Friedmann Laboratory Publishing, St. Petersburg, 1994. 
E. S. Fradkin, D. M. Gitman, and Sh. M. Shvartsman. Quantum electrodynamics with unstable vacuum. Springer, 1991. Berlin, Germany: Springer (1991) 288 p. (Springer series in nuclear and particle physics).

W. Greiner, B. Muller, and J. Rafelski. QUANTUM ELECTRODYNAMICS OF STRONG FIELDS. Springer, 1985. Berlin, Germany: Springer ( 1985) 594 P. ( Texts and Monographs In Physics).

[67] Jan Ambjorn, R. J. Hughes, and N. K. Nielsen. Action principle of bogolyubov coefficients. Ann. Phys., 150:92, 1983.

Jan Ambjorn and Richard J. Hughes. Particle creation in color electric fields. Phys. Lett., B113:305, 1982.

Jan Ambjorn and Richard J. Hughes. Canonical quantization in nonabelian background fields. 1. Ann. Phys., 145:340, 1983.

[68] A. I. Vainshtein, Valentin I. Zakharov, V. A. Novikov, and Mikhail A. Shifman. Calculations in external fields in qcd: An operator method. (in russian). Sov. J. Nucl. Phys., 39:77, 1984.

[69] Martin Reuter, Michael G. Schmidt, and Christian Schubert. Constant external fields in gauge theory and the spin 0,1/2,1 path integrals. Annals Phys., 259:313-365, 1997.

[70] G. K. Savvidy. Infrared instability of the vacuum state of gauge theories and asymptotic freedom. Phys. Lett., B71:133, 1977.

N. K. Nielsen and P. Olesen. An unstable yang-mills field mode. Nucl. Phys., B144:376, 1978.

C. Ragiadakos. A stable symmetrized savvidy vacuum. Phys. Lett., B100:471, 1981.

[71] Asim Yildiz and Paul H. Cox. Vacuum behavior in quantum chromodynamics. Phys. Rev., D21:1095, 1980.

M. Claudson, A. Yildiz, and P. H. Cox. Vacuum behavior in quantum chromodynamics. ii. Phys. Rev., D22:2022-2026, 1980.

Stephen L. Adler. An overrelaxation method for the monte carlo evaluation of the partition function for multiquadratic actions. Phys. Rev., D23:2901, 1981.

Walter Dittrich and Martin Reuter. Effective qcd lagrangian with zeta function regularization. Phys. Lett., B128:321, 1983.

Curt A. Flory. A selfdual gauge field, its quantum fluctuations, and interacting fermions. Phys. Rev., D28:1425, 1983.

Y. M. Cho and D. G. Pak. Dynamical symmetry breaking and magnetic confinement in qcd. hep-th, 2000.

[72] Steven K. Blau, Matt Visser, and Andreas Wipf. Analytical results for the effective action. Int. J. Mod. Phys., A6:5409-5433, 1991.

[73] Abhijit Bhattacharyya et al. Cosmological qcd phase transition and dark matter. Nucl. Phys., A661:629-632, 1999.

[74] Gouranga C. Nayak and Peter van Nieuwenhuizen. Soft-gluon production due to a gluon loop in a constant chromo-electric background field. Phys. Rev., D71:125001, 2005. 
[75] Dmitri Kharzeev, Eugene Levin, and Kirill Tuchin. Multi-particle production and thermalization in high-energy qcd. Phys. Rev., C75:044903, 2007.

[76] E. M. Serebryanyi. Vacuum polarization by magnetic flux: The aharonov-bohm effect. Theor. Math. Phys., 64:846-855, 1985.

[77] Pawel Gornicki. Aharonov-bohm effect and vacuum polarization. Ann. Phys., 202:271-296, 1990.

[78] B.M. Barbashov. JETP, 48:607, 1965.

I. A. Batalin and E. S. Fradkin. Quantum electrodynamics in external fields. 1. Teor. Mat. Fiz., 5:190-218, 1970.

Marc Henneaux and Claudio Teitelboim. Relativistic quantum mechanics of supersymmetric particles. Ann. Phys., 143:127, 1982.

Alexander M. Polyakov. GAUGE FIELDS AND STRINGS. CHUR, SWITZERLAND: HARWOOD, 1987.

A. V. Marshakov and V. Ya. Fainberg. Representation of the propagator of a dirac particle in an external gravitational field as a sum over paths. JETP Lett., 47:565-568, 1988.

V. Ya. Fainberg and A. V. Marshakov. A propagator for fermionic string. Phys. Lett., B211:81, 1988.

V.Ya. Fainberg and A.V. Marshakov. Proc. Phian, 201, 1990.

Jan Ambjorn, Bergfinnur Durhuus, and Thordur Jonsson. A random walk representation of the dirac propagator. Nucl. Phys., B330:509, 1990.

J. Grundberg, T. H. Hansson, and A. Karlhede. Geometrical phases from spinning particles. Phys. Rev., D41:2642, 1990.

G. P. Korchemsky. Quantum geometry of the dirac fermions. Phys. Lett., B232:334, 1989.

J. Grundberg, T. H. Hansson, and A. Karlhede. On polyakov's spin factors. Nucl. Phys., B347:420-440, 1990.

[79] D. M. Gitman and A. V. Saa. Quantization of spinning particle with anomalous magnetic momentum. Class. Quant. Grav., 10:1447-1460, 1993.

[80] G. P. Korchemsky. Quantum geometry of dirac fermions. Int. J. Mod. Phys., A7:339-380, 1992.

T. M. Aliev, V. Ya. Fainberg, and N. K. Pak. Path integral for spin: A new approach. Nucl. Phys., B429:321-343, 1994.

J. W. van Holten. Propagators and path integrals. Nucl. Phys., B457:375-407, 1995.

A. I. Karanikas and C. N. Ktorides. Particle path integral approach to the study of dirac spin 1/2 field systems. Phys. Rev., D52:5883-5897, 1995.

Bodo Geyer, Dmitry Gitman, and Ilya L. Shapiro. Path integral and pseudoclassical action for spinning particle in external electromagnetic and torsion fields. Int. J. Mod. Phys., A15:3861$3876,2000$. 
[81] A. Barducci, R. Casalbuoni, and L. Lusanna. Classical scalar and spinning particles interacting with external yang-mills fields. Nucl. Phys., B124:93, 1977.

[82] S. K. Wong. Field and particle equations for the classical yang-mills field and particles with isotopic spin. Nuovo Cim., A65S10:689-694, 1970.

[83] M. Dresden and S. F. Chen. Solitons, gauge theories and the 'great einstein theorem'. Physica, $83 \mathrm{~A}: 1,1975$.

[84] Rainer Dick. Vector and scalar confinement in gauge theory with a dilaton. Phys. Lett., B409:321-324, 1997.

Rainer Dick. Confinement from a massive scalar in QCD. Eur. Phys. J., C6:701-703, 1999.

M. Chabab, R. Markazi, and E. H. Saidi. On the confining potential in $4 \mathrm{~d} \operatorname{su}(\mathrm{n}(\mathrm{c}))$ gauge theory with dilaton. Eur. Phys. J., C13:543-549, 2000.

[85] Michael B. Green, J. H. Schwarz, and Edward Witten. SUPERSTRING THEORY. Cambridge University Press, 1987. Cambridge, Uk: Univ. Pr. ( 1987) ( Cambridge Monographs On Mathematical Physics).

[86] A. O. Barut and R. Raczka. THEORY OF GROUP REPRESENTATIONS AND APPLICATIONS. Singapore, Singapore: World Scientific ( 1986) 717p.

[87] Y. Ohnuki and T. Kashiwa. Coherent states of fermi operators and the path integral. Prog. Theor. Phys., 60:548, 1978.

[88] D. M. Gitman and I. V. Tyutin. Quantization of fields with constraints. Springer, 1990. Berlin, Germany: Springer (1990) 291 p. (Springer series in nuclear and particle physics).

[89] D. M. Gitman and I. V. Tyutin. Hamiltonization of theories with degenerate coordinates. Nucl. Phys., B630:509-527, 2002.

[90] W. Greiner, S. Schramm, and E. Stein. Quantum chromodynamics. Berlin, Germany: Springer (2002) $551 \mathrm{p}$.

[91] A. N. Vasiliev. Functional methods in quantum field theory and statistical physics. Gordon and Breach Science Publishers, 1986. Amsterdam, Netherlands: Gordon and Breach (1998) $312 \mathrm{p}$.

[92] R. Jackiw and S. Y. Pi. Covariant coordinate transformations on noncommutative space. Phys. Rev. Lett., 88:111603, 2002.

[93] Paolo Aschieri et al. A gravity theory on noncommutative spaces. Class. Quant. Grav., 22:3511-3532, 2005.

[94] B. M. Zupnik. Reality in noncommutative gravity. Class. Quant. Grav., 24:15-26, 2007.

[95] M. Chaichian, P. P. Kulish, K. Nishijima, and A. Tureanu. On a lorentz-invariant interpretation of noncommutative space-time and its implications on noncommutative qft. Phys. Lett., B604:98-102, 2004.

[96] Julius Wess. Deformed coordinate spaces: Derivatives. 2003. 
[97] S. Cacciatori et al. Noncommutative gravity in two dimensions. Class. Quant. Grav., 19:40294042, 2002.

[98] Dimitri V. Vassilevich. Quantum noncommutative gravity in two dimensions. Nucl. Phys., B715:695-712, 2005.

[99] D. V. Vassilevich. Constraints, gauge symmetries, and noncommutative gravity in two dimensions. Theor. Math. Phys., 148:928-940, 2006.

[100] Amir Masoud Ghezelbash and Shahrokh Parvizi. Gauged noncommutative Wess-ZuminoWitten models. Nucl. Phys., B592:408-416, 2001.

[101] M. Henneaux and C. Teitelboim. Quantization of gauge systems. Princeton, USA: Univ. Pr. (1992) $520 \mathrm{p}$.

[102] D. M. Gitman and I. V. Tyutin. General quadratic gauge theory: Constraint structure, symmetries, and physical functions. J. Phys., A38:5581, 2005.

[103] Robert Marnelius. THE LAGRANGIAN AND HAMILTONIAN FORMULATION OF RELATIVISTIC PARTICLE MECHANICS. Phys. Rev., D10:2535, 1974.

[104] J. Llosa and J. Vives. Hamiltonian formalism for nonlocal lagrangians. J. Math. Phys, 35:2856, 1994.

[105] R. P. Woodard. A canonical formalism for Lagrangians with nonlocality of finite extent. Phys. Rev., A62:052105, 2000.

[106] Joaquim Gomis, Kiyoshi Kamimura, and Josep Llosa. Hamiltonian formalism for space-time non-commutative theories. Phys. Rev., D63:045003, 2001.

[107] J. David Brown. LOWER DIMENSIONAL GRAVITY. SINGAPORE, SINGAPORE: WORLD SCIENTIFIC (1988) 152p.

[108] S. M. (Ed. ) Christensen. Quantum theory of gravity. xvi, 483p, 1984.

[109] Ali H. Chamseddine and D. Wyler. TOPOLOGICAL GRAVITY IN (1+1)-DIMENSIONS. Nucl. Phys., B340:595-616, 1990.

[110] K. Isler and C. A. Trugenberger. A GAUGE THEORY OF TWO-DIMENSIONAL QUANTUM GRAVITY. Phys. Rev. Lett., 63:834, 1989.

[111] D. Grumiller, W. Kummer, and D. V. Vassilevich. Dilaton gravity in two dimensions. Phys. Rept., 369:327-430, 2002.

[112] Edward Witten. On string theory and black holes. Phys. Rev., D44:314-324, 1991.

[113] Gautam Mandal, Anirvan M. Sengupta, and Spenta R. Wadia. Classical solutions of twodimensional string theory. Mod. Phys. Lett., A6:1685-1692, 1991.

[114] S. Elitzur, A. Forge, and E. Rabinovici. Some global aspects of string compactifications. Nucl. Phys., B359:581-610, 1991. 
[115] Curtis G. Jr. Callan, E. J. Martinec, M. J. Perry, and D. Friedan. Strings in Background Fields. Nucl. Phys., B262:593, 1985.

[116] M. O. Katanaev, W. Kummer, and H. Liebl. Geometric interpretation and classification of global solutions in generalized dilaton gravity. Phys. Rev., D53:5609-5618, 1996.

[117] W. Kummer, H. Liebl, and D. V. Vassilevich. Exact path integral quantization of generic 2-d dilaton gravity. Nucl. Phys., B493:491-502, 1997.

[118] W. Kummer, H. Liebl, and D. V. Vassilevich. Integrating geometry in general 2d dilaton gravity with matter. Nucl. Phys., B544:403-431, 1999.

[119] D. Grumiller, W. Kummer, and D. V. Vassilevich. The virtual black hole in 2d quantum gravity. Nucl. Phys., B580:438-456, 2000.

[120] D. Grumiller, W. Kummer, and D. V. Vassilevich. A note on the triviality of kappadeformations of gravity. Ukr. J. Phys., 48:329-333, 2003.

[121] Fedele Lizzi, Sachindeo Vaidya, and Patrizia Vitale. Twisted conformal symmetry in noncommutative two- dimensional quantum field theory. Phys. Rev., D73:125020, 2006.

[122] Vincent Rivasseau. Non-commutative renormalization. 2007.

[123] R. Gurau, J. Magnen, V. Rivasseau, and A. Tanasa. A translation-invariant renormalizable non-commutative scalar model. 2008.

[124] H. O. Girotti, M. Gomes, Victor O. Rivelles, and A. J. da Silva. A consistent noncommutative field theory: The Wess-Zumino model. Nucl. Phys., B587:299-310, 2000.

[125] Nathan Seiberg. Noncommutative superspace, $\mathrm{N}=1 / 2$ supersymmetry, field theory and string theory. JHEP, 06:010, 2003.

[126] Marija Dimitrijevic, Voja Radovanovic, and Julius Wess. Field Theory on Nonanticommutative Superspace. JHEP, 12:059, 2007.

[127] Yoshishige Kobayashi and Shin Sasaki. Non-local Wess-Zumino model on nilpotent noncommutative superspace. Phys. Rev., D72:065015, 2005.

[128] A. A. Zheltukhin. Supersymmetric Lorentz invariant deformations of superspaces. Mod. Phys. Lett., A21:2117-2132, 2006.

[129] D. M. Gitman and D. V. Vassilevich. Space-time noncommutativity with a bifermionic parameter. Mod. Phys. Lett., A23:887-893, 2008.

[130] D. M. Gitman, A. E. Goncalves, and I. V. Tyutin. New pseudoclassical model for Weyl particles. Phys. Rev., D50:5439-5442, 1994.

[131] Raimar Wulkenhaar. Non-renormalizability of theta-expanded noncommutative qed. JHEP, 03:024, 2002.

[132] Michael Edward Peskin and Daniel V. Schroeder. An Introduction to quantum field theory. Reading, USA: Addison-Wesley (1995) 842 p. 
[133] M. Hayakawa. Perturbative analysis on infrared aspects of noncommutative QED on $\mathrm{R}^{* *} 4$. Phys. Lett., B478:394-400, 2000.

[134] M. Hayakawa. Perturbative analysis on infrared and ultraviolet aspects of noncommutative qed on $\mathrm{r}^{* *} 4.1999$.

[135] J. S. Dowker and Raymond Critchley. Effective Lagrangian and Energy Momentum Tensor in de Sitter Space. Phys. Rev., D13:3224, 1976.

[136] S. W. Hawking. Zeta Function Regularization of Path Integrals in Curved Space-Time. Commun. Math. Phys., 55:133, 1977.

[137] D. V. Vassilevich. Heat kernel expansion: User's manual. Phys. Rept., 388:279-360, 2003.

[138] Dmitri V. Vassilevich. Heat Trace Asymptotics on Noncommutative Spaces. SIGMA, 3:093, 2007.

[139] Dmitri V. Vassilevich. Non-commutative heat kernel. Lett. Math. Phys., 67:185-194, 2004.

[140] Victor Gayral and Bruno Iochum. The spectral action for Moyal planes. J. Math. Phys., 46:043503, 2005.

[141] Dmitri V. Vassilevich. Heat kernel, effective action and anomalies in noncommutative theories. JHEP, 08:085, 2005.

[142] V. Gayral, B. Iochum, and D. V. Vassilevich. Heat kernel and number theory on NC-torus. Commun. Math. Phys., 273:415-443, 2007.

[143] Peter B. Gilkey. The Spectral geometry of a Riemannian manifold. J. Diff. Geom., 10:601-618, 1975 .

[144] Maxim Kontsevich. Deformation quantization of Poisson manifolds, I. Lett. Math. Phys., $66: 157-216,2003$.

[145] Giuliano M. Gavazzi. Weyl-ordered fermions and path integrals. J. Math. Phys., 30, December 1989. 